University of Rhode Island

DigitalCommons@URI

Open Access Master's Theses

2014

\title{
AN INVESTIGATION INTO GROUND EFFECT FOR AN UNDERWATER BIOLOGICALLY INSPIRED FLAPPING FOIL
}

Paulstephen Chierico

University of Rhode Island, pstusn@gmail.com

Follow this and additional works at: https://digitalcommons.uri.edu/theses

\section{Recommended Citation}

Chierico, Paulstephen, "AN INVESTIGATION INTO GROUND EFFECT FOR AN UNDERWATER BIOLOGICALLY INSPIRED FLAPPING FOIL" (2014). Open Access Master's Theses. Paper 429. https://digitalcommons.uri.edu/theses/429

This Thesis is brought to you for free and open access by DigitalCommons@URI. It has been accepted for inclusion in Open Access Master's Theses by an authorized administrator of DigitalCommons@URI. For more information, please contact digitalcommons-group@uri.edu. 
AN INVESTIGATION INTO GROUND EFFECT FOR

AN UNDERWATER BIOLOGICALLY INSPIRED

FLAPPING FOIL

BY

PAULSTEPHEN CHIERICO

A THESIS SUBMITTED IN PARTIAL FULFILLMENT OF THE

REQUIREMENTS FOR THE DEGREE OF

MASTER OF SCIENCE

IN

OCEAN ENGINEERING

UNIVERSITY OF RHODE ISLAND

2014 
MASTER OF SCIENCE DEGREE THESIS

OF

\section{PAULSTEPHEN CHIERICO}

\section{APPROVED:}

Thesis Committee:

Major Professor: Stephen C. Licht

Jason M. Dahl

Musa K. Jouaneh

Nasser H. Zawia

DEAN OF THE GRADUATE SCHOOL

UNIVERSITY OF RHODE ISLAND

2014 
Abstract

The topic that was investigated was whether there is a lift and thrust benefit to flapping an underwater, biologically inspired three dimensional foil near the solid bottom surface (so that it was in ground effect). The experimental method used a dual canister device that allowed actuation in roll and pitch, with force sensors attached to the pitch shaft, in order to record the forces produced by the foil as it flapped. The dual canister was towed on a carriage at a constant speed in a large tow tank that had been configured to have a long run of constant, deep water depth, a very short transition period, and then another long run of constant, shallow water depth. Due to this configuration, in one run the foil was able to encounter freestream conditions and ground effect conditions. The results proved in all sixteen experimental cases for varying Strouhal number and maximum angle of attack that the mean lift coefficient near the bottom was larger than that in the freestream. A potentially useful data point, for which the mean thrust coefficient had a positive change from flapping near bottom as compared to the freestream, was found to have a change in maximum instantaneous lift force of $14 \%$. This would give a large enough change in signal strength that it could be used as a parameter on a future underwater vehicle to control altitude above the ground. The benefit to flapping in ground effect was equivalent to a larger than $1^{\circ}$ pitch bias difference at a zero mean lift coefficient. Additionally, there was a thrust benefit seen to flapping in ground effect, but only under certain kinematics. Though not as dramatic as the benefit in lift, there was still an $8 \%$ difference in the mean thrust coefficient observed between flapping near the bottom and flapping in the freestream, for the case where the largest change in 
mean thrust coefficient was observed. This could equate to a large savings in battery life, and hence a longer endurance for a vehicle taking advantage of the thrust benefit seen by flapping in ground effect.

While this work remains preliminary in nature, it shows that much more useful work remains to be done to explore the benefit induced by flapping a foil close to a hard surface ground. 
Acknowledgements

I would first like to acknowledge that this research topic has been inspired by the flapping fins and wings of swimming and flying animals. While most of the literature in the topic of bioinspiration and biomimetics ascribes the abilities of these amazing animals to evolution, I personally believe in God and creation. I have faith that God is the Genius who created the world in all its wonder for His glory and our enjoyment. "Then God said, "Let the waters abound with an abundance of living creatures, and let birds fly above the earth across the face of the firmament of the heavens." So God created great sea creatures and every living thing that moves, with which the waters abounded, according to their kind, and every winged bird according to its kind. And God saw that it was good." (Genesis 1:20-21)

Secondly, I would like to honor and give thanks for my wife, Peggy Ann Chierico. After ten years of marriage I realize more and more each day that I could never be successful even in the smallest thing if I did not have her constant loving support and encouragement. I also thank my parents, Paul and Vera Chierico for their love and support. They both have a passion for learning, with a strong sense of adventure and fun, which I have inherited.

Third, I acknowledge all of the military veterans who have gone before me, enabling me and my family to live and enjoy freedom in this great constitutionbased federal republic of ours. I thank the United States Navy, and the Civil Engineer Corps for giving me this fantastic opportunity to earn a post-graduate education. 
Fourth, I thank Professor Stephen Licht for his help and guidance. What was astonishing to me is that such a talented and accomplished individual sought me out to work on his research team when I first met him!

Fifth, I would like to thank all of those who helped me out with this project. I definitely stood on the shoulders of the giant, Sam Rauworth, and would not have been able to do any of this without his help. I additionally thank Matt Perkins, Alexandre Fossat, Jordan Kirby, and Robin Freeland for their knowledge and oftentimes manual labor. 
Table of Contents

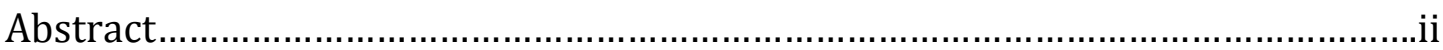

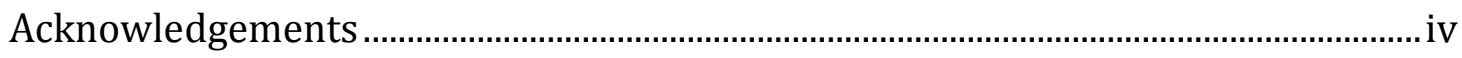

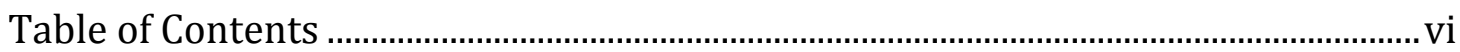

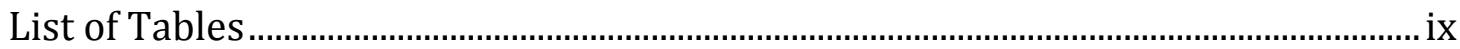

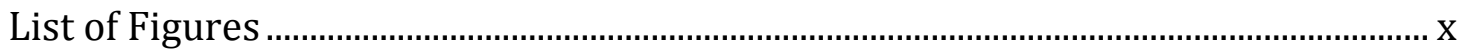

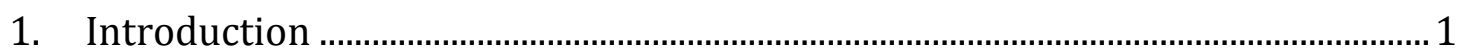

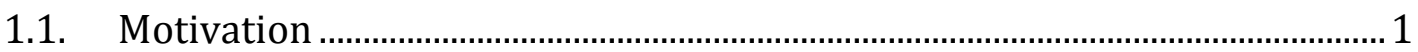

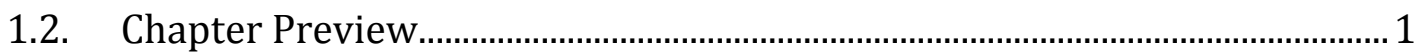

1.3. Background and Literature Review ............................................................... 2

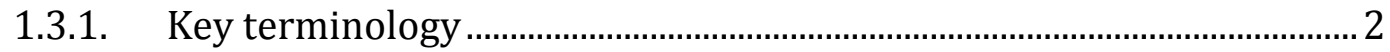

1.3.2. Ground effect for fixed airfoils ................................................................. 7

1.3.3. Animal flight aerodynamics in ground effect.......................................... 8

1.3.4. Ground effect on an infinite foil............................................................. 9

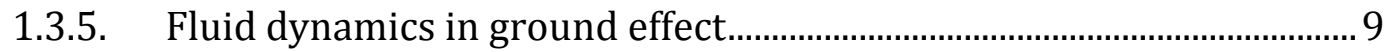

1.3.6. Swimming near the substrate ................................................................ 11

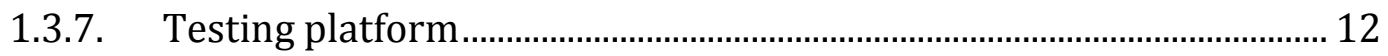

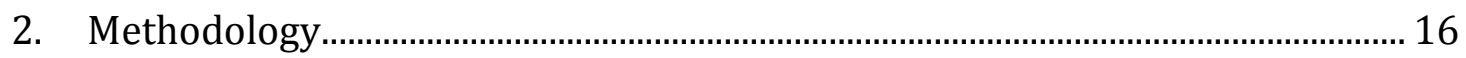

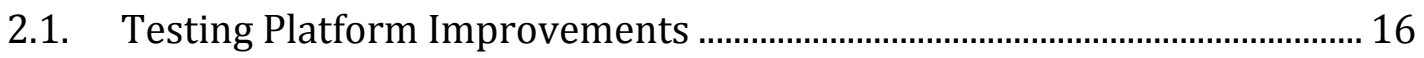

2.1.1. Install Force Sensors with Larger Separation......................................... 16 


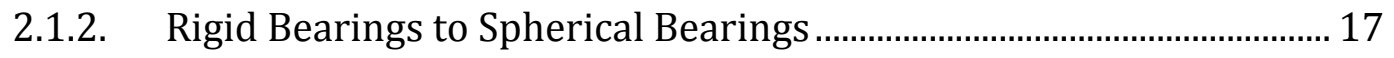

2.1.3. Split plate design ........................................................................................... 18

2.1.4. Wiring Improvements................................................................................ 19

2.1.5. Calibration Improvements .................................................................... 22

2.2. Experimental Method .................................................................................. 33

3. Results and Discussion .............................................................................................. 41

3.1. Establishing zero mean lift coefficient ............................................................. 41

3.2. Contour plots ................................................................................................ 42

3.3. Results for different series of Strouhal number ............................................ 49

3.4. Results for a single maximum angle of attack ............................................. 54

3.5. Phase averaged lift and thrust force plots .................................................... 57

4. Design Improvements and Error Analysis ............................................................ 62

4.1. Design Improvements................................................................................. 62

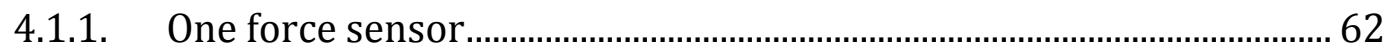

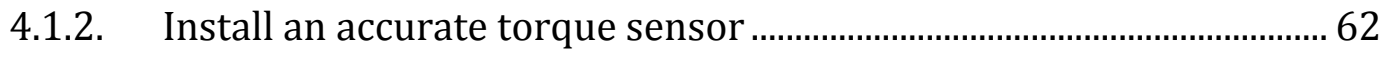

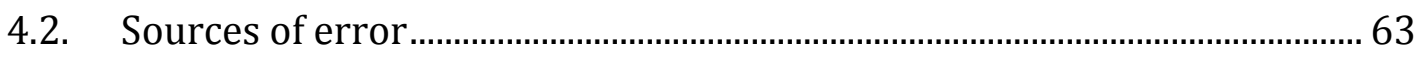

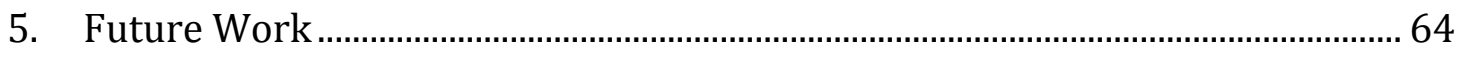

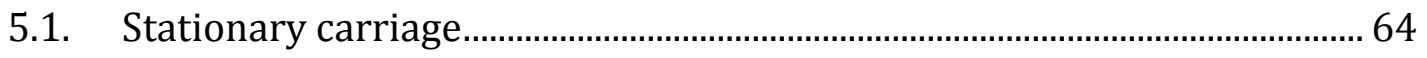

5.2. Under a free water surface ………………………………………………... 64

5.3. Under wave conditions................................................................................... 64 


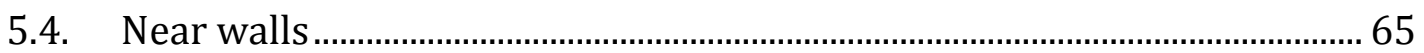

5.5. Performance and operational considerations................................................. 65

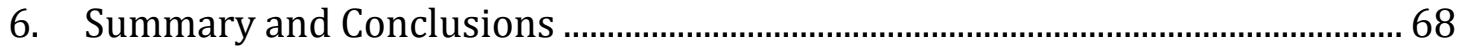

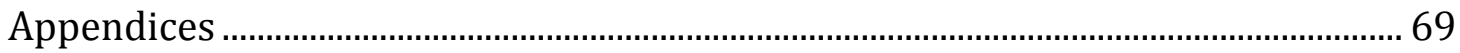

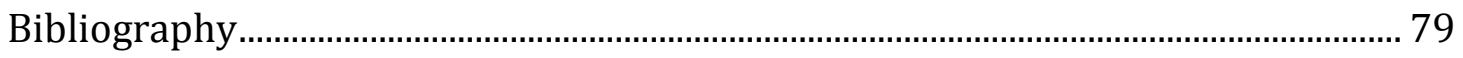




\section{List of Tables}

Table 1. Experimental matrix. 
List of Figures

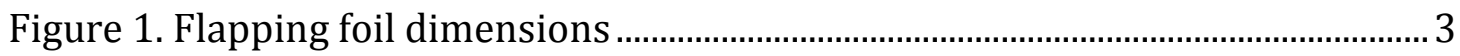

Figure 2. Reverse vortex street behind a swimming fish (Eloy, 2012) ........................ 3

Figure 3. Angle of attack at one span location (Polidoro, 2003)................................... 6

Figure 4. Instantaneous vorticity contours for $\mathrm{H} / \mathrm{c}=3$ (freestream) .......................... 10

Figure 5. Instantaneous vorticity contours for $\mathrm{H} / \mathrm{c}=1$ (near ground) ......................... 11

Figure 6. Plan view of testing platform (Rauworth, 2014) ....................................... 13

Figure 7. Profile view of testing platform (Rauworth, 2014) ...................................... 13

Figure 8. Phase averaged lift and thrust plot example (Rauworth, 2014) ............... 15

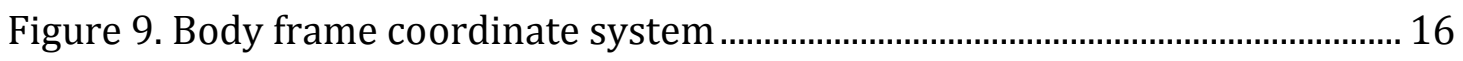

Figure 10. Clamped vs. pinned pitch shaft support bearings........................................ 17

Figure 11. Comparison between old (large metal) and new (small plastic)

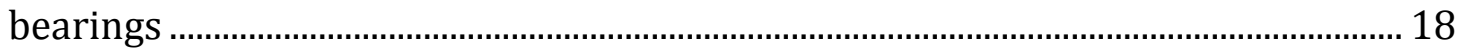

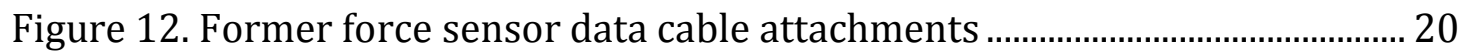

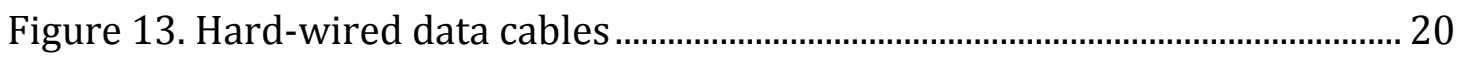

Figure 14. Weights hung from set of six notches (Rauworth, 2014) ......................... 22

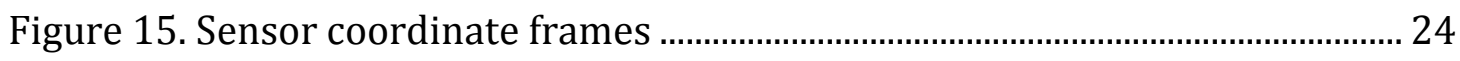

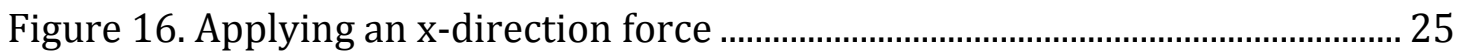

Figure 17. Applying a z-direction force ……............................................................ 26

Figure 18. Applying simultaneous y- and z-direction forces ..................................... 27

Figure 19. Sensor responses due to applied $x$-force....................................................... 29

Figure 20. Sensor responses due to applied z-force ..................................................... 29

Figure 21. Sensor responses due to applied y- and z-forces ......................................... 29 
Figure 22. Sensor responses due to applied y-force

Figure 23. Winches ready to move the metal plate seams of the beach 34

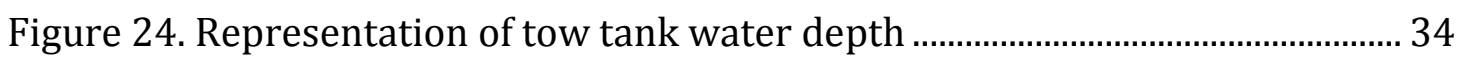

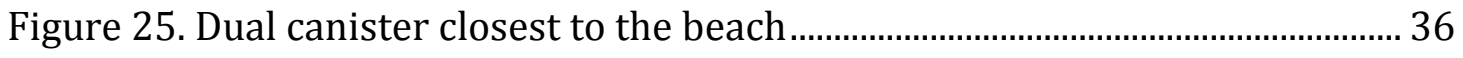

Figure 26. Side view of dual canister near bottom ....................................................... 36

Figure 27. Three view drawing to represent pitch canister......................................... 39

Figure 28. Mean lift coefficient as a function of pitch bias............................................ 41

Figure 29. Contour plot for mean lift coefficient in freestream (H/c=8.0) .............. 44

Figure 30. Contour plot for mean lift coefficient near bottom $(\mathrm{H} / \mathrm{c}=1.1)$............... 44

Figure 31. Contour plot for change in mean lift coefficient.......................................... 45

Figure 32. Contour plot for mean thrust coefficient in freestream $(\mathrm{H} / \mathrm{c}=8.0) \ldots . . .47$

Figure 33. Contour plot for mean thrust coefficient near bottom $(\mathrm{H} / \mathrm{c}=1.1)$......... 47

Figure 34. Contour plot for change in mean thrust coefficient .................................48

Figure 35. Change in mean lift coefficient as a function of maximum angle of attack

Figure 36. Change in maximum lift force as a function of maximum angle of attack 50

Figure 37. Change in minimum lift force as a function of maximum angle of attack

Figure 38. Change in mean thrust coefficient as a function of maximum angle of attack

Figure 39. Change in maximum thrust force as a function of maximum angle of attack 
Figure 40. Change in minimum thrust force as a function of maximum angle of attack 54

Figure 41. Change in mean lift coefficient or mean thrust coefficient as a function of Strouhal number for maximum angle of attack of $30^{\circ}$ 55

Figure 42. Change in minimum lift force or maximum lift force as a function of Strouhal number for maximum angle of attack of $30^{\circ}$ 56

Figure 43. Change in minimum thrust force or maximum thrust force as a function of Strouhal number for maximum angle of attack of $30^{\circ}$ 56

Figure 44. Phase averaged lift and thrust force for $S t=0.3$ and $\alpha \max =30^{\circ}$ (dashed=freestream, solid=near bottom)

Figure 45. Phase averaged lift and thrust force for $S t=0.4$ and $\alpha \max =30^{\circ}$ (dashed=freestream, solid=near bottom) 58

Figure 46. Phase averaged lift and thrust force for $\mathrm{St}=0.5$ and $\alpha \max =30^{\circ}$ (dashed=freestream, solid=near bottom) 59 Figure 47. Comparison between lift and thrust plot for (Rauworth, 2014) and this work 60

Figure 48. Phase averaged lift and thrust force for $\mathrm{St}=0.6$ and $\alpha \max =30^{\circ}$ (dashed=freestream, solid=near bottom) 61 


\section{Introduction}

\subsection{Motivation}

Aquatic animals that employ flapping fins for underwater propulsion, such as turtles, fish, and penguins, are endowed with amazing abilities that engineers have yet to even match in our unmanned underwater vehicles (UUV). These animals are able to gracefully swim, navigate, avoid obstacles, avoid predators, and catch prey in complex underwater environments. Some of these complex underwater environments include areas of interest that humans greatly desire to operate UUVs in. There are many reasons why flapping foil UUVs would need to operate close to the bottom surface of a body of water such as the ocean floor. Navigation would be improved and the adverse effects of waves and currents would be minimized. Military UUVs could take advantage of the stealth that it provides, as well as simply the opportunity to closely inspect long stretches of pipeline and communications cabling. In the future, fin propelled vehicles will be able to use real time fin force data to estimate, and hence control, altitude near the bottom surface (Licht \& Dahl, 2013). This work is a humble beginning to investigating the benefits and challenges that may be observed by flapping in ground effect.

\subsection{Chapter Preview}

The rest of this chapter includes background and literature review sections, which describe key terms related to flapping foils. Chapter 2 presents the methodology of this work, including improvements that were made to the testing platform that was used, and the experimental method. Chapter 3 
describes the results that were obtained, presenting the data in a variety of ways, and discusses the findings. Chapter 4 suggests further design improvements that are recommended, and presents some error analysis. Chapter 5 proposes some future work that may be done with the testing platform with respect to ground effect. Chapter 6 gives the summary and conclusions from this work. Appendices and a bibliography follow chapter 6 .

\subsection{Background and Literature Review}

\subsubsection{Key terminology}

\subsubsection{Foil Dimensions}

The flapping foil that has been used in this work is one of the four that were employed on Finnegan the RoboTurtle, described in (Licht, 2008). It has been made from a titanium framework surrounded by Shore 80A polyurethane elastomer, in a biologically inspired shape that emulates a turtle fin. The chord (c) is the distance from leading edge to trailing edge. Since it is not a rectangular planform, the chord that will be used is $0.1 \mathrm{~m}$, which is the mean chord. In this work the mean chord will always be referred to as simply "chord". The span $(s)$ is the distance from root to tip, $0.4 \mathrm{~m}$. The cross section of the fin is symmetrical about its chord, meaning that it is not cambered. At its thickest, the foil is $0.015 \mathrm{~m}$ thick. The cross section approximates a NACA 0012 shape with a rounded leading edge and a sharp trailing edge. Foil dimensions are depicted below. Because of the polyurethane elastomer it is constructed from, and the placement of the titanium framework, one-third of the chord closest to the trailing edge is 
compliant. This property has not been quantified nor studied in the current work.

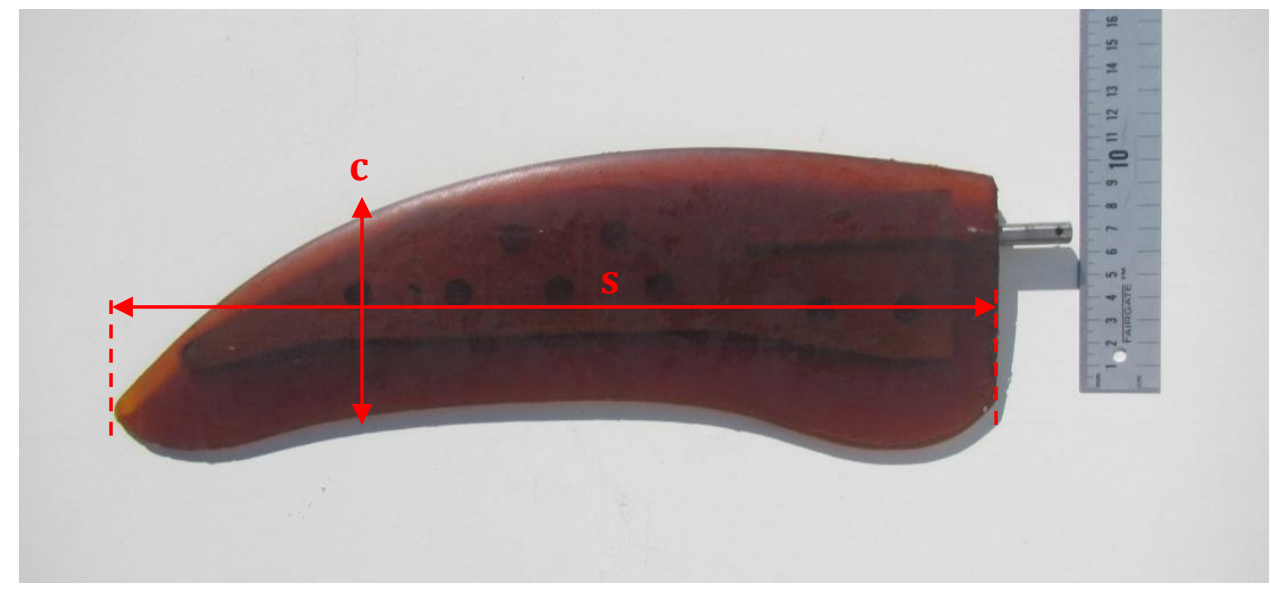

Figure 1. Flapping foil dimensions

\subsubsection{Non dimensional parameters}

It is well known that a foil oscillating in heave and pitch can produce a mean forward thrust force, through the generation of a reversed von Karman vortex street. This is the principle by which many swimming and flying animals propel themselves (Streitlien \& Triantafyllou, 1997). The depiction below shows a reversed vortex street generated behind a fish swimming by flapping its caudal fin (tail), as seen from above.

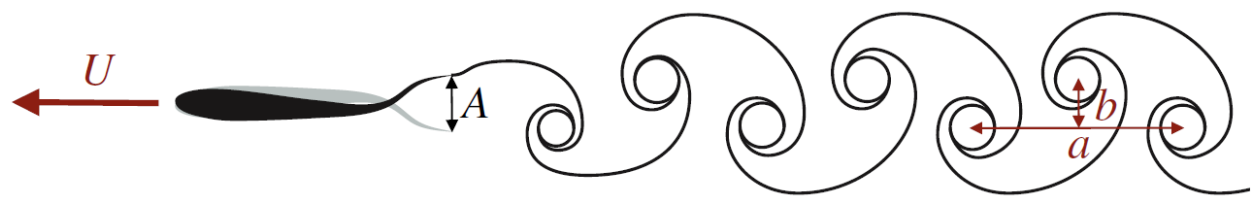

Figure 2. Reverse vortex street behind a swimming fish (Eloy, 2012)

Where $U$ is the forward speed, $A$ is the peak-to-peak amplitude of the tail flap, and $b / a$ is the vortex spacing ratio. This same pattern is generated by the flapping of 
pectoral fins on fish using labriform motion (Sfakiotakis, Lane, \& Davis, 1999), and by the fins on turtles. When averaged over one or more periods of motion in time, the vortex street has the velocity profile of a jet, with zero mean lift force, and some non-zero mean thrust force.

The first important non dimensional parameter is the Strouhal number $(S t)$, a ratio used to characterize the vortex pattern as it relates the vehicle velocity to the frequency and size of each vortex generated. In this work, St has been defined as:

$$
S t=\frac{f \cdot A}{U}=\frac{f \cdot 2 r_{0.7} \cdot \varphi_{0}}{U}
$$

\section{Equation 1. Definition of Strouhal number}

Where $f$ is the flapping frequency, $r_{0.7}$ will be addressed below, and $\varphi_{0}$ is the roll amplitude. For a two dimensional foil, the amplitude, A, is clear. However, for the three dimensional case it is less clear what distance should be used. Following (Techet, 2008), the amplitude to base St upon is taken as the amplitude of an arc length at $70 \%$ of the foil span, away from the root. The location was selected to be consistent with conventional propeller notations and for easy comparison with past flapping foil experiments. The value $r_{0}$ is the distance from the roll axis to the root of the fin. The radius of the $70 \%$ span location of the foil is denoted as $\mathrm{r}_{0.7}$ with the roll axis as the origin:

$$
r_{0.7}=r_{0}+0.7 s
$$

\section{Equation 2. Radius of $70 \%$ span location}


Following (Polidoro, 2003), the location of $\mathrm{r}_{0.7}$ is very close to the effective center of hydrodynamic force on the foil. For this reason in the current work, $\mathrm{r}_{0.7}$ is assumed to be the location of the hydrodynamic center of the foil, the location at which the lift forces and thrust forces generated by the entire fin is taken to act through. This location is $0.28 \mathrm{~m}$ away from the root, and $0.12 \mathrm{~m}$ away from the tip. One final note is that St in Equation 1 is based on the amplitude of $\mathrm{r}_{0.7}$, and not based on the peak-to-peak amplitude of the fin tip.

The next important non dimensional parameter is the heave to chord ratio $\left(h_{0.7} / c\right)$. This ratio is the heave amplitude divided by the chord length, and is based on the heave amplitude at the assumed hydrodynamic center.

Lastly, height above ground to chord ratio $(H / c)$ will be used to characterize when the foil is in ground effect. For $\mathrm{H} / \mathrm{c}$ of greater than 3 , the effects of being close to the ground are negligible (Wu, Shu, Zhao, \& Yan, 2014). So by that definition, ground effect may be expected for $\mathrm{H} / \mathrm{c}<3$.

\subsubsection{Foil Kinematics}

A few more equations and figures must be introduced in order to characterize the foil kinematics (Polidoro, 2003). Roll motion is described by:

$$
\varphi(t)=\varphi_{0} \sin (\omega t)
$$

\section{Equation 3. Equation for roll of the foil}

Where $\omega$ is the flapping frequency expressed in radians. Similarly, pitch motion can be described by:

$$
\theta(t)=\theta_{0} \cos (w t)
$$


Where $\theta_{0}$ is the pitch amplitude. In this work the phase angle $(\psi)$ between roll and pitch will always be $\pi / 2$, such that maximum pitch occurs at zero roll, and zero pitch occurs at maximum roll. The angle of attack encountered by the foil at each point along the span has two components. The first is due to the ratio of forward velocity and heave velocity, and the second is due to the instantaneous pitch position of the foil:

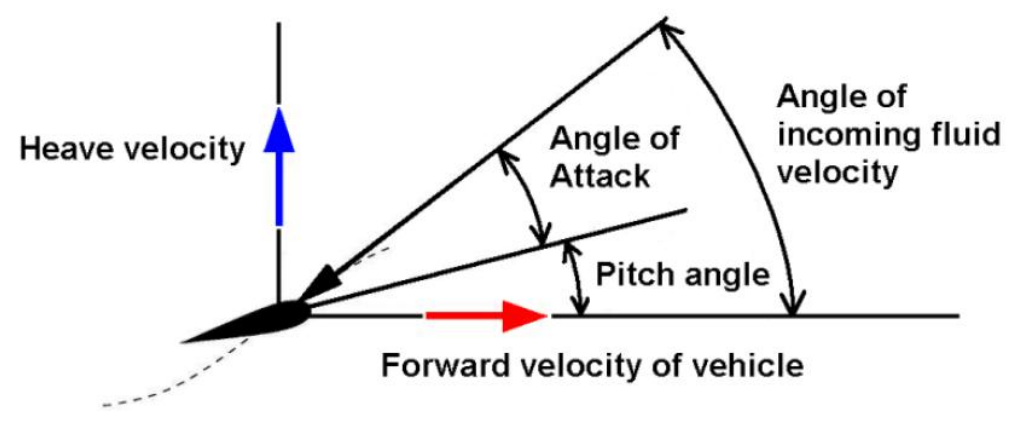

Figure 3. Angle of attack at one span location (Polidoro, 2003)

Therefore the angle of attack is described by:

$$
\alpha(t)=-\arctan \left(\frac{\omega r_{0.7} \varphi_{0} \cos (\omega t)}{U}\right)+\theta_{0} \cos (\omega t)+\theta_{\text {bias }}
$$

Equation 5. Equation for angle of attack of the foil

The first term represents the roll induced angle of attack and the second two terms represents the pitch induced angle of attack. For the majority of motions that produce thrust, the pitch motion is selected to reduce the maximum angle of attack $\left(\alpha_{\max }\right)$. The angle of attack is a function of spanwise location, but again the maximum angle of attack is a parameter that is calculated at the assumed hydrodynamic center of the foil, as seen in the equation above. 
As a performance metric, the mean lift and mean thrust coefficients will be used later on in analysis. The mean lift coefficient is found by the following:

$$
\bar{C}_{L}=\frac{2 \bar{L}}{\rho U^{2} S C}
$$

\section{Equation 6. Equation for mean lift coefficient}

Where $\rho$ is the water density. Similarly, the mean thrust coefficient is:

$$
\bar{C}_{T}=\frac{2 \bar{T}}{\rho U^{2} S C}
$$

\section{Equation 7. Equation for mean thrust coefficient}

For any given geometry the St and $\alpha_{\max }$, when taken together, are the only things needed to completely describe the foil motion (Polidoro, 2003).

\subsubsection{Ground effect for fixed airfoils}

To start the literature review section, the first paper that will be discussed relates to a simple case in aerodynamics using a fixed airfoil, moving close to the ground. Ground effect is well understood in aerodynamics for fixed airfoils. (Garcia \& Katz, 2003) described trapped vortices between fixed airfoils and the ground surface, giving insight into their application on racecars. The principle of increasing fluid dynamic loads (lift) by creating strong vortices near solid surfaces existed in nature long before attempts were made to understand its mechanics. It is natural, therefore, that vorticity is frequently used to explain an airfoil's lift, which is often called "bound vorticity" (to separate it from "unbound" vortices found in wakes). Consequently, augmentation of the fluid dynamic loads by adding unbound (or trapped) vortices is a logical extension to the "more 
vorticity, more lift" principle. The authors concluded that the trapped vortex is a viable principle for lift augmentation, and immediately moved to apply that to ground vehicles, in particular racecars. Ground vehicles, contrary to aircraft, move close to the ground, and their incidence relative to the freestream falls into the small angle of attack (or zero incidence) category. The utilization of this type of vortex lift, therefore, becomes quite attractive. For example, such vortices when trapped beneath a moving automobile and the ground can increase the vehicle's negative lift (downforce). This aerodynamic downforce is often exploited by racecar designers in order to increase tire adhesion and vehicle high speed performance.

\subsubsection{Animal flight aerodynamics in ground effect}

In this next paper, the author studied flying animals, making general observations about their performance flying in ground effect. Again, fixed wings were used in this study. (Rayner, 1991) developed a complete theory for a fixed wing in ground effect, based on a steady state lifting-line wing model, and uses it to show how ground effect is likely to affect the flight performance of an animal close above a surface. The most important findings were that flight in ground effect above a flat, smooth surface may give an animal considerable performance advantages, including a reduction in cost of transport of up to $15 \%$, and a reduction in mechanical flight power of as much as 35\%, compared with values for flight out of ground effect. The author additionally concluded that slow flight performance in ground effect is very poor, owing to the horizontal air velocity induced around the wing in the presence of the ground. Ground effect was defined in that work as the situation when an animal or an aircraft flies close 
above a plane surface; the aerodynamic properties of its wings are altered as a result of the interaction of the vortices on the wing and the wake with the surface. Ground effect was noted to have a significant effect on flight performance during takeoff and landing. The dominant effect is a reduction in the induced (vortex) drag, and hence a saving in the thrust required for level flight. In the current study it would be interesting to find a similar thrust benefit.

\subsubsection{Ground effect on an infinite foil}

Moving away from both fixed wings and aerospace applications, the next paper conducted preliminary experimental studies in force production by heaving and pitching oscillating (two dimensional) foils in ground effect in a small water tow tank (Licht \& Dahl, 2013). They showed that the mean lift for a typical set of thrust generating foil kinematics when operating within two chord lengths of the bottom is increased by an amount consistent with approximately a 1 degree positive (away from wall) bias in the foil pitch angle. Additionally, they found that flapping a foil near a solid boundary generates significant (18\%) variation in peak magnitude of downstroke vs. upstroke instantaneous lift. In this work, there is a dual canister mechanism that enables the foil motion. This also closely simulates a vehicle body. In Licht and Dahl's work only the foil was in the flow and the driving mechanism was outside. Additionally, their foil span was vertical and flapped near a wall, but in this work the foil is flapped near a ground. This is bound to create differences in the magnitude of the effect that is observed in the two data sets.

1.3.5. Fluid dynamics in ground effect 
The next work numerically studied insect wing flapping in air. (Wu, Shu, Zhao, \& Yan, 2014) investigated ground effect on a flapping insect wing in forward flight, through simulation using an Immersed Boundary-Lattice Boltzmann Method (IB-LBM). A NACA 0012 airfoil, which models the insect wing cross-section, was considered. The airfoil executed a combined motion of harmonic heave and pitch rotation. The Reynolds number and the amplitude of motion were fixed, while the height above ground and frequency of oscillation were examined. They found that the flow patterns shed from the foil were altered due to the ground effect. The following two figures show the instantaneous vorticity contours under different Strouhal numbers. Looking at flow patterns alone, the authors concluded that there was negligible difference at an $\mathrm{H} / \mathrm{c}>3$, which is used as the basis for freestream conditions. In these figures, the solid line represents counterclockwise vorticity and the dashed line represents clockwise vorticity.

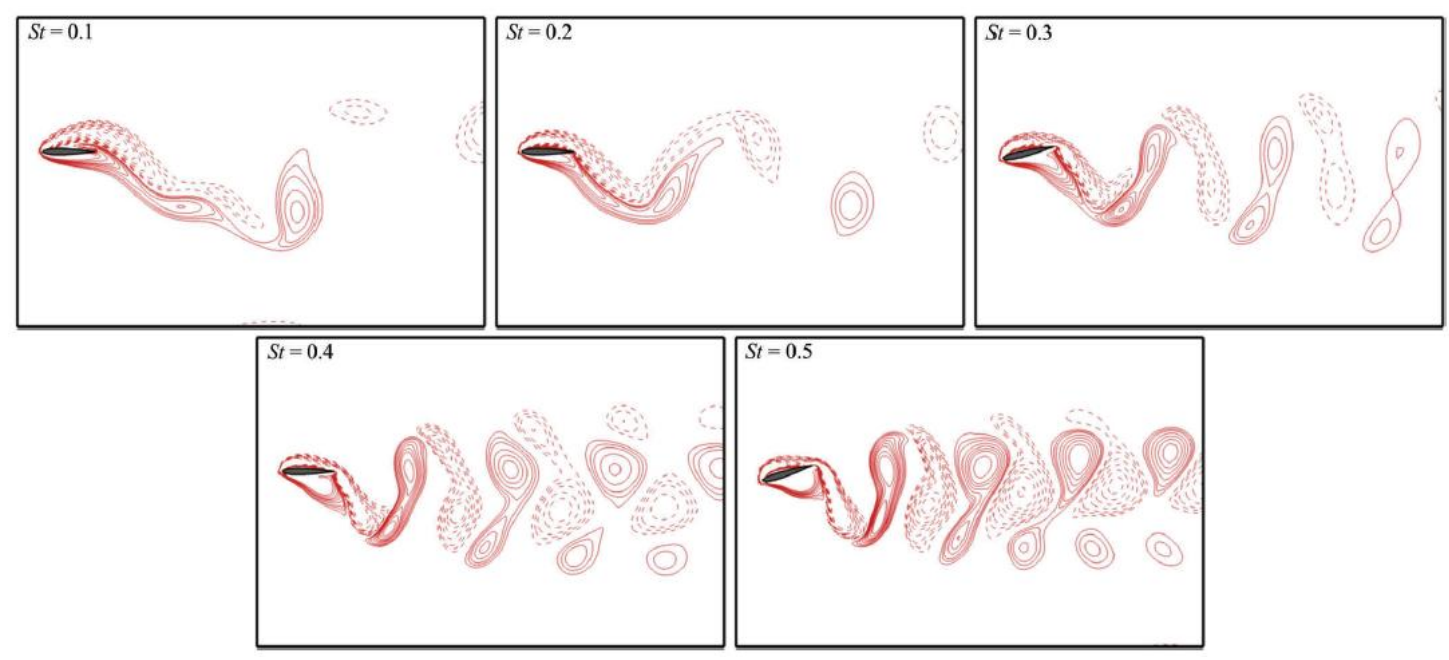

Figure 4. Instantaneous vorticity contours for $\mathrm{H} / \mathrm{c}=3$ (freestream)

(Wu, Shu, Zhao, \& Yan, 2014) 


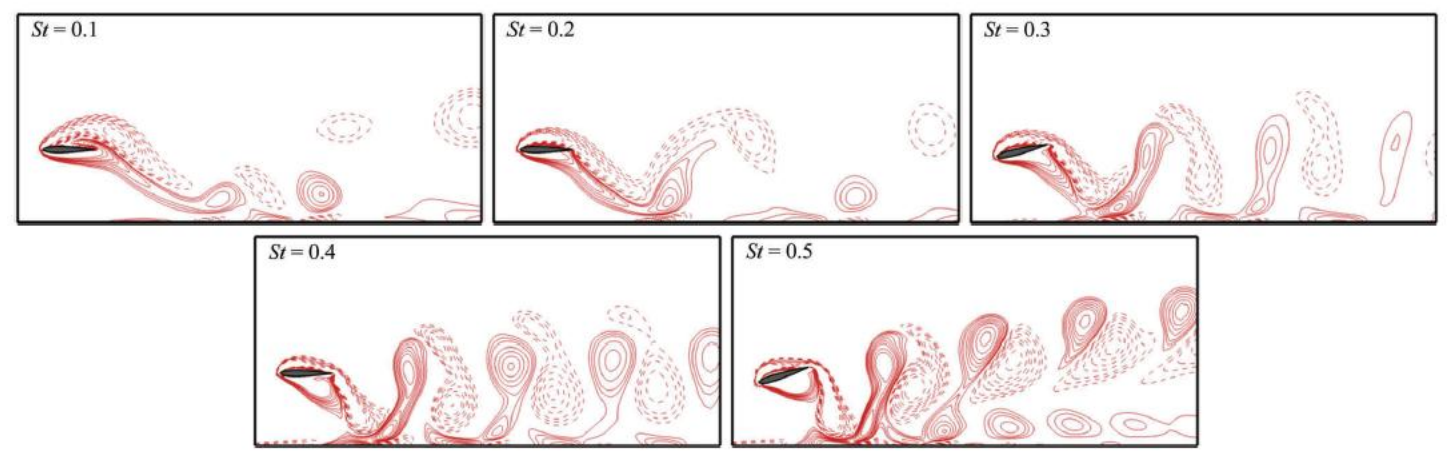

Figure 5. Instantaneous vorticity contours for $\mathrm{H} / \mathrm{c}=1$ (near ground) (Wu, Shu, Zhao, \& Yan, 2014)

Note that the vortex interaction with the ground is very significant at $H / c=1$, which changes the vortex structures in the wake. At low $\mathrm{St}(\mathrm{St}=0.1$ and 0.2 ), the size of the vortex shed from the flapping foil is shortened due to the presence of ground. However, the strength of vortices is increased, which may be attributed to vortex interaction with the ground. As St keeps increasing ( $\mathrm{St}=0.3$ and 0.4 ), the vortex interaction becomes stronger and stronger, gradually affecting vortex shedding of the foil. When $\mathrm{St}=0.5$, it is most evident that the vortices have been compressed to an oblate shape. Consequently, there is a notable angle between the ground and the center line of the vortex street. The authors relate this angle to changes in the overall mean lift vector direction, resulting in an increased mean lift coefficient seen in ground effect. The authors also noted that the mean drag coefficient was increased at low frequency (low St) and decreased at high frequency (high St), i.e., the mean thrust coefficient would decrease for low St and increase at high St.

\subsubsection{Swimming near the substrate}

Having just looked at a numerical simulation which investigated ground effect, the next report to be introduced is the most relevant to the present work. 
In (Blevins \& Lauder, 2013) a simple, stingray-inspired physical model was used to give insight into ground effects on undulatory swimmers, contrasting the selfpropelled swimming speed, power requirements, and hydrodynamics of fins swimming with fixed kinematics near and far from a solid boundary. The authors experimentally determined that contrary to findings for gliding birds and other fixed-wing fliers, ground effect does not necessarily enhance the performance of undulating fins. Under most kinematic conditions, fins do not swim faster in ground effect, power requirements increase, and the cost of transport can increase by up to $10 \%$. The influence of ground effect varies with kinematics, suggesting that benthic fish might modulate their swimming behavior to minimize locomotor penalties and incur benefits from swimming near a substrate. In this work a recirculating tank was used and the flow speed was altered to match the thrust produced by the moving fin. When the fin maintains an equilibrium position, thrust and drag are balanced during each cycle of motion. Although the experimental setup was different, the results from this study will prove to be valuable in the current work.

\subsubsection{Testing platform}

The current effort extends and builds on (Rauworth, 2014) wherein a system capable of testing submerged underwater flapping foils in a tow tank while recording force and position data was created. The existing testing platform consisted of: a dual canister system to enable roll and pitch of a foil, an aluminum carriage attachment assembly, an instrument chassis, a power supply, a laser distance measurement (LDM) device on the tow tank carriage, and a computer on the shore to both control the dual canister system and to record the 
data from the force sensors. It is this testing platform, as well as the experimental techniques and processing codes that the author developed, that has been used in the current work. The following two figures show pictorial representations of the testing platform:

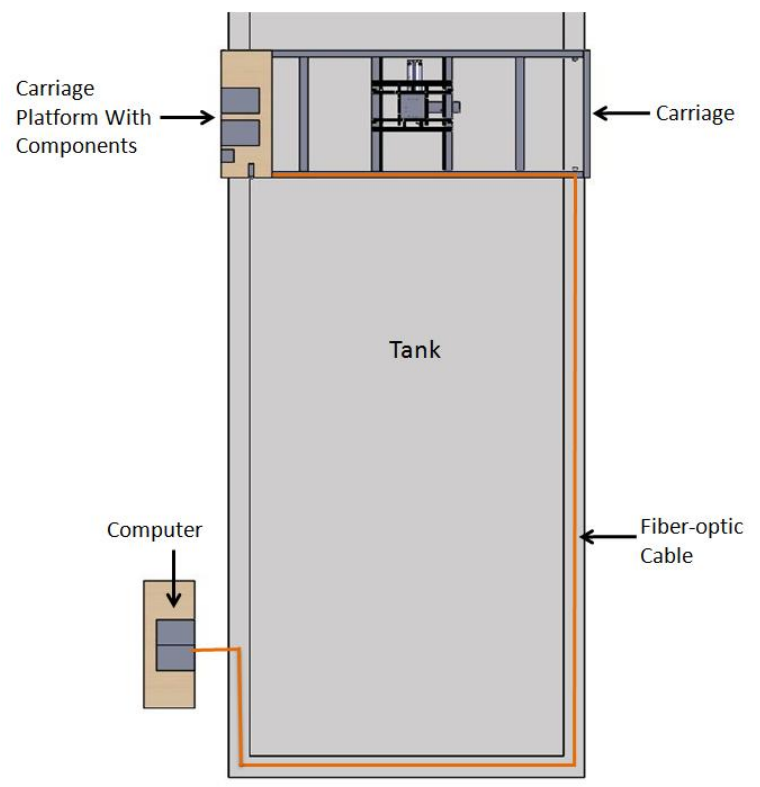

Figure 6. Plan view of testing platform (Rauworth, 2014)

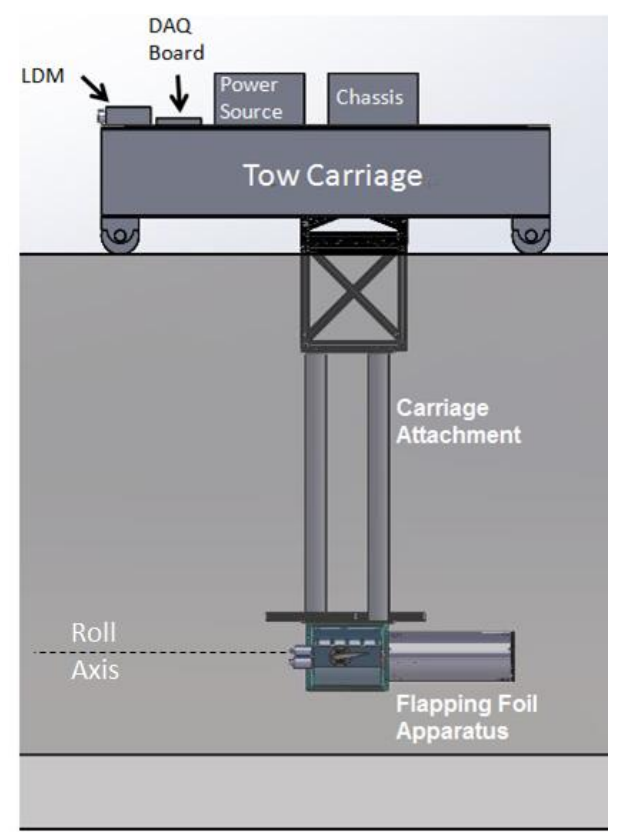

Figure 7. Profile view of testing platform (Rauworth, 2014) 
In addition to setting all of the above listed components up and getting them to work together, the author also compared his newly generated data to that of past testing conducted. For a comparison of mean thrust coefficient contour plots, Rauworth used (Techet, 2008). In that work, a linearly tapered three dimensional foil was used with the same dual canister apparatus, a recirculating tank, and a six-axis, waterproof strain gauge sensor. The sensor measured the three force components and the three moment components on the foil. Measured forces, mean force coefficients, and hydrodynamic efficiency data were presented. Mean thrust coefficient contours from (Techet, 2008) and (Rauworth, 2014) closely matched in shape and trend, but the values were different (due to different foils and sensing methods used). For a comparison of time sequenced lift and thrust forces, Rauworth used (Polidoro, 2003). The two displayed similar trends and waveforms when compared. Rauworth found that phase averaged data adheres to expected theoretical results and a representative figure is shown below, for $\mathrm{St}=0.5$ and $\alpha_{\max }=30^{\circ}$. It is included to summarize the author's findings and as an example to compare the results of the current work with in a later section: 


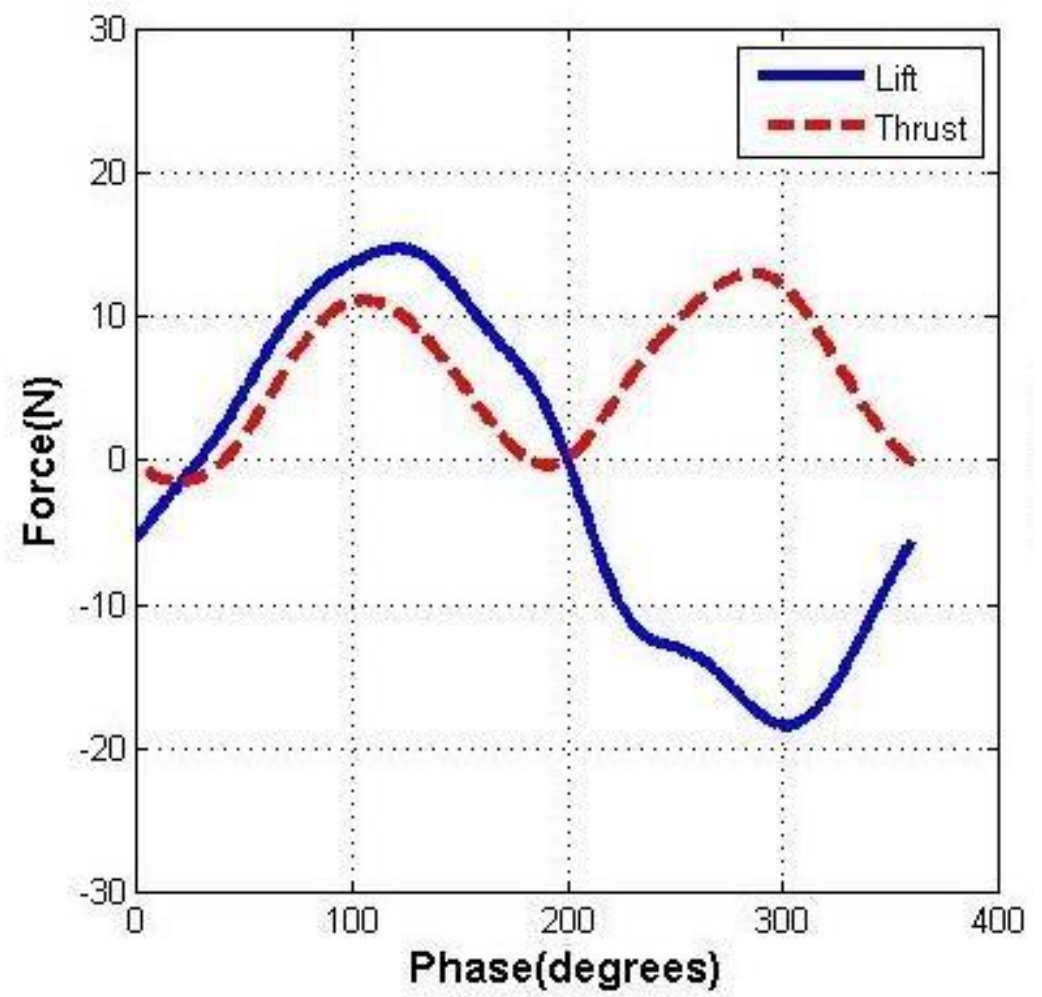

Figure 8. Phase averaged lift and thrust plot example (Rauworth, 2014)

Finally, in chapter 5 of (Rauworth, 2014), he suggested design improvements that should be made to the testing platform, and virtually every component has been improved upon in the current work. 
2. Methodology

2.1. Testing Platform Improvements

The following figure shows the body frame coordinate system of the dual canister. Notice that the origin of the coordinate system is established at the intersection of the roll axis and the pitch shaft.

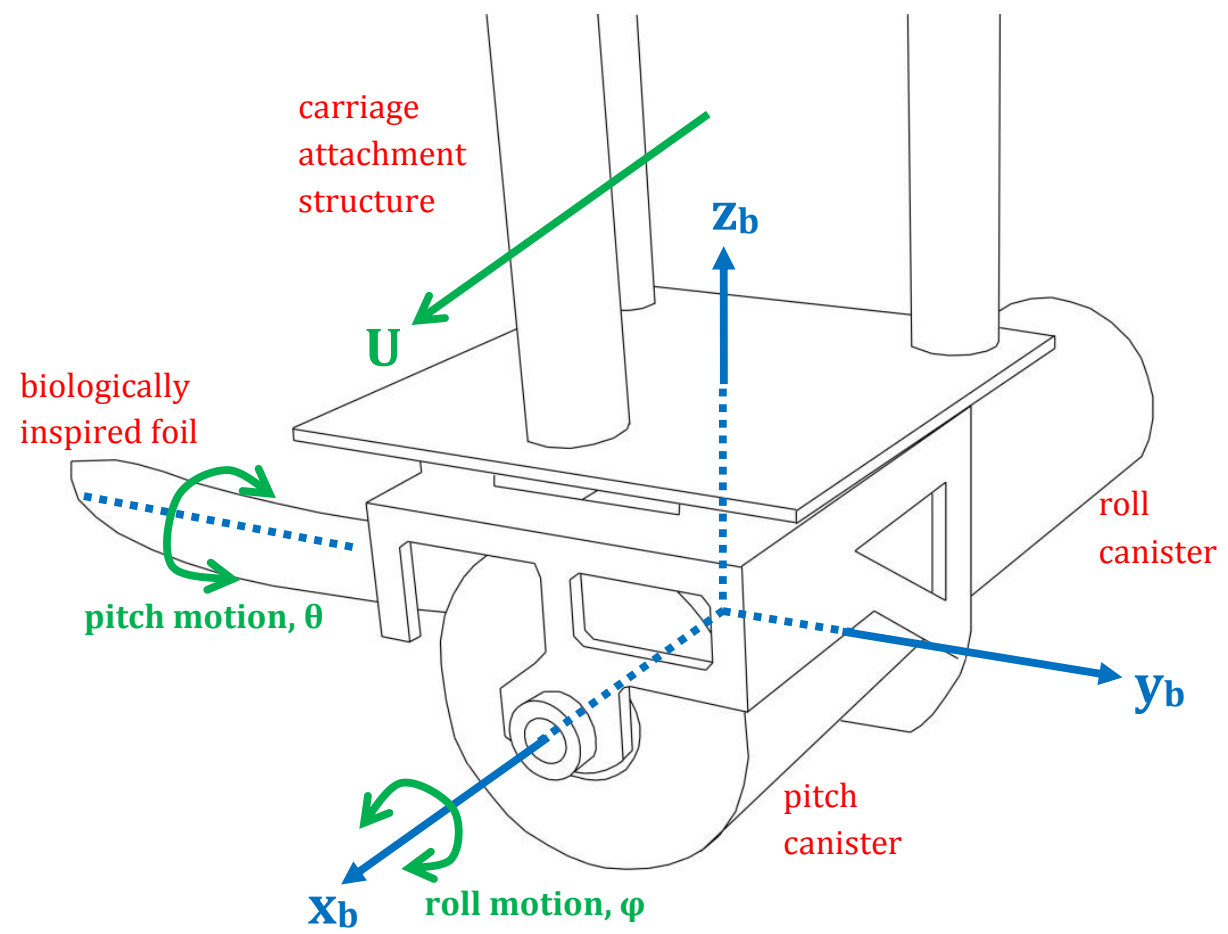

Figure 9. Body frame coordinate system

\subsubsection{Install Force Sensors with Larger Separation}

The two Kistler type 9602 three-axis force sensors are attached via bearings to the pitch shaft. The lift and thrust forces on the foil are transmitted to the force sensors, which send the recorded forces (sampled at a rate of $200 \mathrm{~Hz}$ ) to the shore based computer. The procedure to use the recorded forces in all threeaxes to find the lift and thrust on the foil is described later in the Data Processing section. The force sensors were previously installed at a y-distance separation of 
$0.03 \mathrm{~m}$, due to design constraints. In the current work, the force sensors are installed at a y-distance separation is $0.092 \mathrm{~m}$, a threefold increase. The desire to increase the separation between the force sensors was driven by the need to improve the signal to noise ratio.

\subsubsection{Rigid Bearings to Spherical Bearings}

The pitch shaft was previously supported by two large, rigid bearings, which only allowed rotation about the pitch axis. Acting as clamped connections, the bearings undoubtedly imparted moments about the body $\mathrm{x}$ - and z-axes. A representation follows, showing first the closely spaced, large, rigid bearings, which clamped around the pitch shaft. Below that shows the spaced out, small, spherical bearings, which act as pinned supports to the pitch shaft.

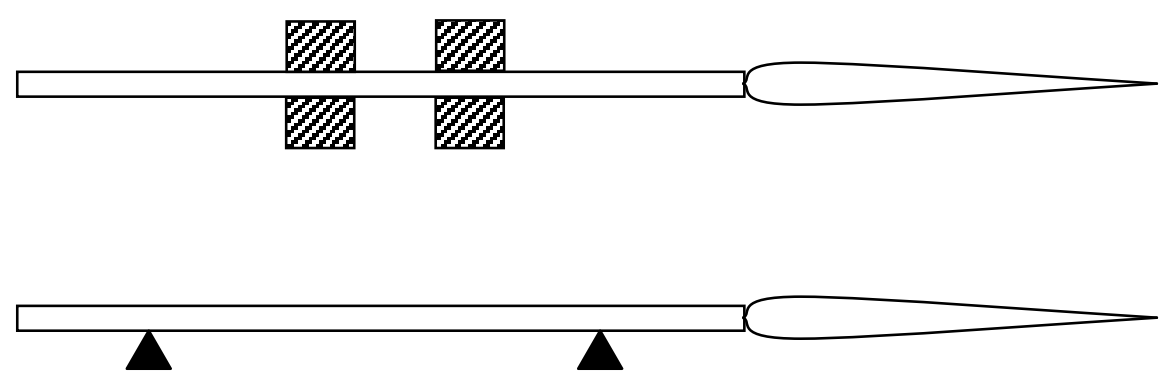

Figure 10. Clamped vs. pinned pitch shaft support bearings

It was imperative to eliminate these additional moments, because they introduced significant errors. The method of obtaining lift and thrust forces for the flapping foil involves using force data from the force sensors, finding the moments of these forces about the $\mathrm{x}$ - and $\mathrm{z}$-axes, and then dividing out a $\mathrm{y}$ distance to the fin assumed hydrodynamic center (equations given in a later section in this chapter). The solution was to replace the rigid bearings with 
spherical bearings, which readily allow rotation about the pitch axis, but are not constrained in rotation about the other two axes.

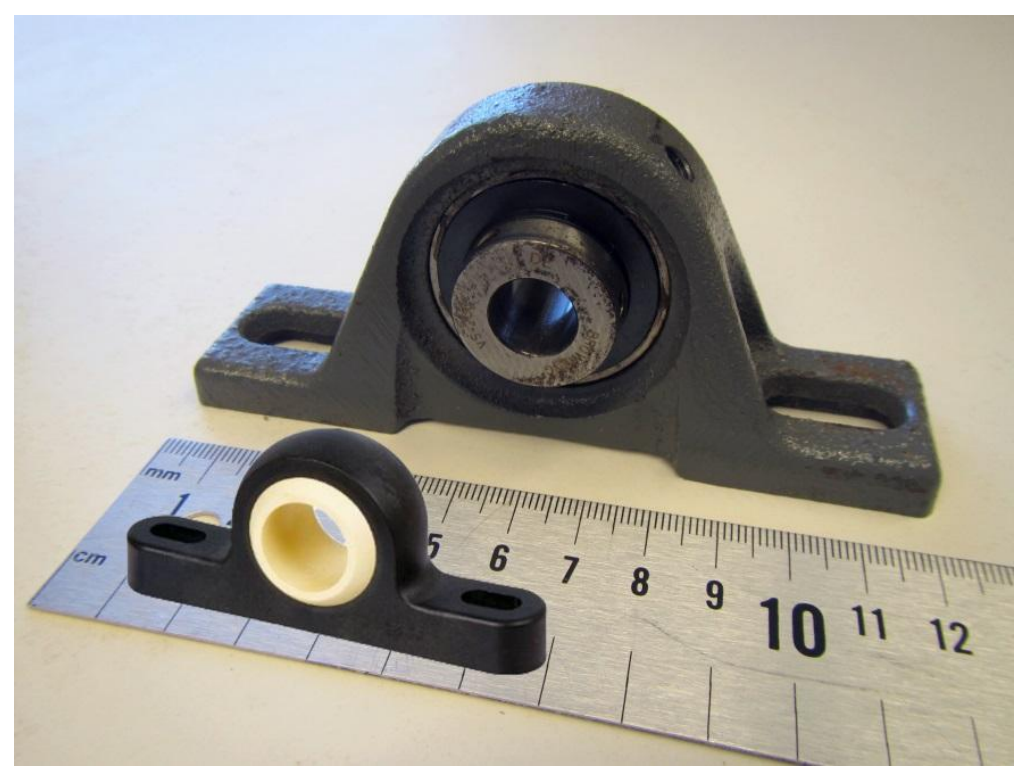

Figure 11. Comparison between old (large metal) and new (small plastic) bearings

The bearings chosen were igubal ${ }^{\mathrm{TM}}$ pillow block bearings from igus incorporated. The new bearings are maintenance free and self-lubricating. The igubal bearings consist of a housing with a spherical plastic insert which freely rotates in any direction inside the housing. Although stiction may be a problem with the new bearings, they will facilitate a far smaller degree of data corruption due to extraneous moments, compared to the previous rigid bearings. An additional benefit to replacing the bearings is a significant space savings in the pitch canister, allowing for easier installation of internal components.

\subsubsection{Split plate design}

The previous method of mounting the two large, rigid bearings had them rigidly mounted onto a solid aluminum plate which rested on top of both force 
sensors, which were on top of another solid aluminum plate secured into the delrin canister housing. The solid plate that connected the force sensors also potentially supported moments, again corrupting the data. The single plate was replaced with two small aluminum plates. The new mounting system is a solid aluminum plate secured into the delrin housing, with the two force sensors above that. On top of force sensor 1 is a small aluminum plate that supports one igubal bearing, the pitch motor, and the pitch homing bracket. On top of force sensor 2 is another small aluminum plate that supports the second igubal bearing. The new mounting system eliminates any solid connection that bridges the gap between the bearings and force sensors. Again, an additional improvement is the space and weight savings of the new system, as well as easier installation.

\subsubsection{Wiring Improvements}

2.1.4.1. Method of connection of dual canister to carriage

Cables conducting the data from the force sensors, through the dual canister, up the carriage attachment to the carriage, used to attach to a National Instruments block (a NI SCB-68A shielded 68-pin connector block for DAQ devices) via rather tenuous four pin connectors that frequently bent or broke. 


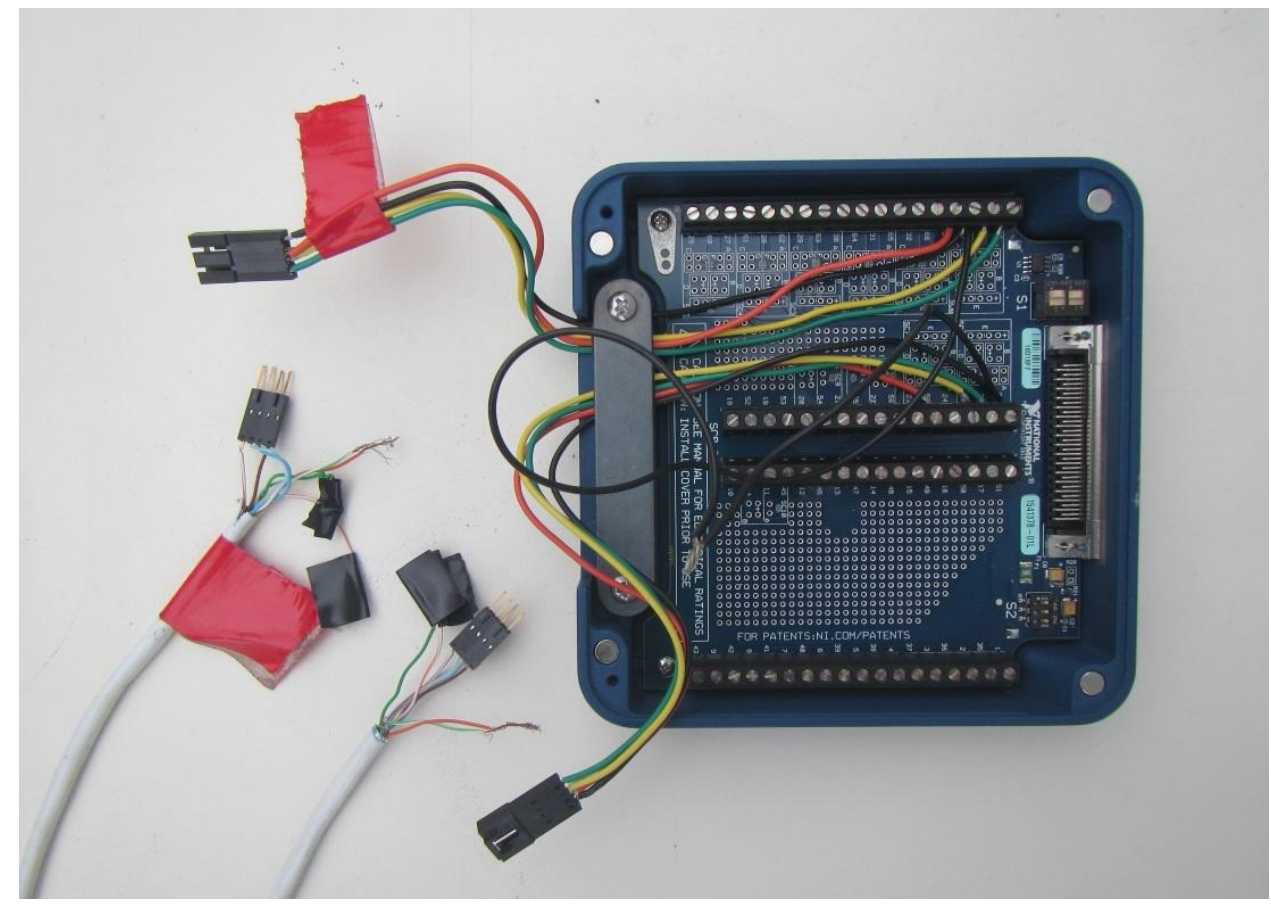

Figure 12. Former force sensor data cable attachments

Rather than having two connections to make each time the system is hooked up to the carriage, many wires were eliminated, and the two cables were hard-wired directly into the NI block.

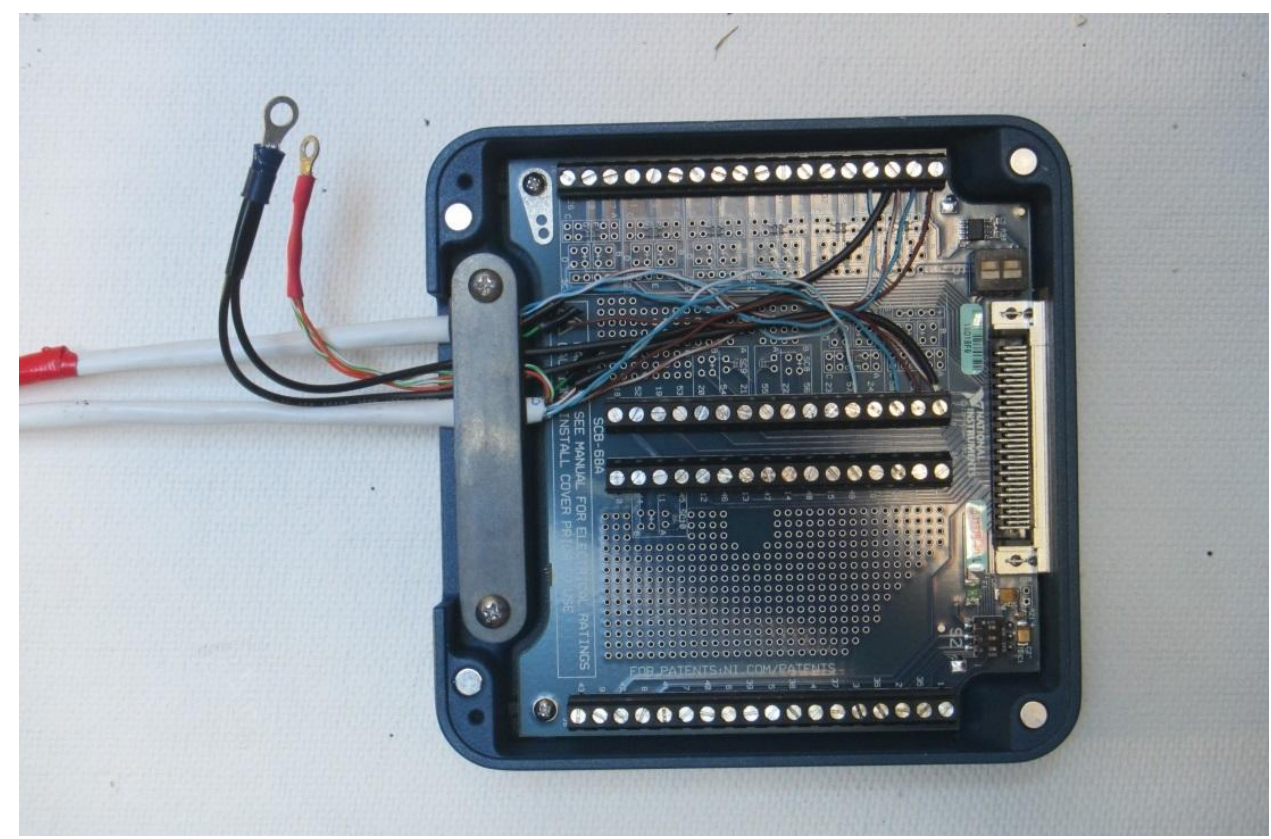

Figure 13. Hard-wired data cables 
The NI block is moved with the dual canister apparatus each time it is removed from the carriage, and connects to the chassis (NI PXIe-1082) via a National Instruments cable.

\subsubsection{Force sensors power supply}

The force sensors had previously been powered via the left hand side of a power supply on the carriage, a BK precision 1673 triple output DC power supply. This required the operator to twist together the solid orange and green/white wires from each force sensor data cable to clip to the red alligator clip, leading to the power source. The black alligator clip from the power source would then clip to black wires that needed to twist together from the NI block. If the operator wanted to re-zero the force sensors, the left hand side voltage dial of the BK power supply was simply turned to zero to power down the force sensors, and then turned back up to $20 \mathrm{~V}$. The new method of providing power to the force sensors is from the chassis, through a NI PXI-4110 programmable DC power supply. Wires go from the chassis to clip to the NI block, and then power is conducted through the force sensor data cables to the force sensors. Now, to rezero the force sensors (which was done at the start of each data collection run), the operator simply flips a switch that is incorporated into the force sensor collection software (LabVIEW 12.0f3).

\subsubsection{LDM power supply}

The LDM used to be powered from the chassis, the $20 \mathrm{~V}$ programmable supply powers the force sensors, as just described. The LDM is now powered via the left hand side of the BK power supply. 


\subsubsection{Dual canister power supply}

Power is supplied to the roll canister via an Impulse waterproof connector. These cables were previously clipped using alligator clips that were plug in leads to the right hand side of the BK power supply. The plug in leads were cut off, and directly soldered to the ends of the red and black cable pair. The negative supply line was grounded to earth/ carriage, resulting in a dramatic decrease in noise recorded at the DAQ card.

\subsubsection{Calibration Improvements}

\subsubsection{Previous calibration procedure}

The previous method of calibration is described in (Rauworth, 2014), chapter 3.2 and chapter 4 . The method used an extra foil that had six notches cut into the leading edge at known distances away from the roll axis. A set of three weights was hung from each notch. The following shows a typical calibration in progress:

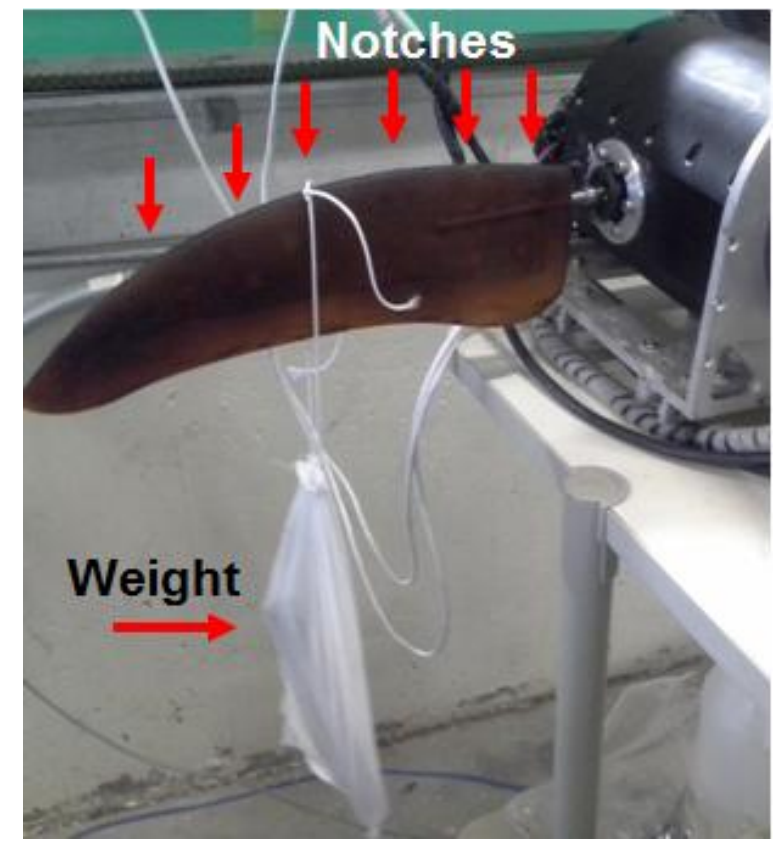

Figure 14. Weights hung from set of six notches (Rauworth, 2014) 
This procedure was repeated for the positive and negative direction for all three coordinate directions. The output was 18 data points for each coordinate direction. For example, if the weight was hung in the positive z-direction, the output was for one quadrant of a chart, with a small response in $\mathrm{x}$, a small response in $\mathrm{y}$, and a large response in $\mathrm{z}$. The large response in $\mathrm{z}$ gave three distinct trend lines for the three weights, but those trend lines when taken as a whole, gave a different straight line. The slope of that line, fit through all 18 points, was found to be the sensitivity of $\mathrm{z}$ for that sensor due to an applied zforce. The cross-terms (sensitivity of $\mathrm{x}$ and $\mathrm{y}$ due to an applied $\mathrm{z}$-force) were small and neglected. In summary, this procedure used a force applied at a moment arm to produce a voltage response at each sensor. Because weights and moment arm distances were all known, each voltage response could be equated to a force that produced it, through a linear relationship.

\subsubsection{New calibration procedure}

Instead of using three weights to apply various moments giving sensor responses, this work used seven weights to apply force in each coordinate axis direction, by hanging the weights directly from each igubal bearing (since the bearings were secured above each force sensor). The entire calibration section of this work is written from the perspective of the sensor frames of reference, unless specified, which are shown below. The sensor directions were defined as shown, notice that they do not adhere to a right hand rule coordinate system. 

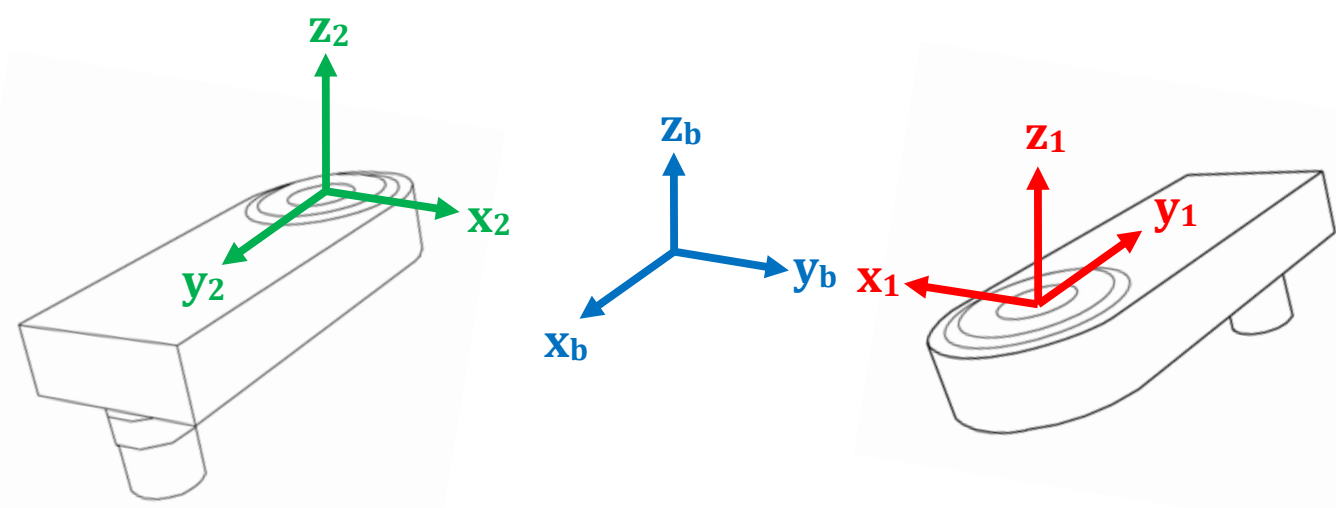

Figure 15. Sensor coordinate frames

As mentioned earlier it was prudent to increase the separation between them and the new installation method required the sensors to be installed as shown in order to accommodate the pitch motor. For reference, the body frame coordinate system is shown in blue. Sensor 1 , on the port side of the dual canister and closer to the body frame origin, is shown in red. Sensor 2, on the starboard side of the dual canister and closer to the foil, is shown in green. For calibration, the $\mathrm{x}$ - and z-coordinate directions were clean and easy to obtain calibration data from. For $x$, the weights were hung directly out the side hole where the foil would be. 


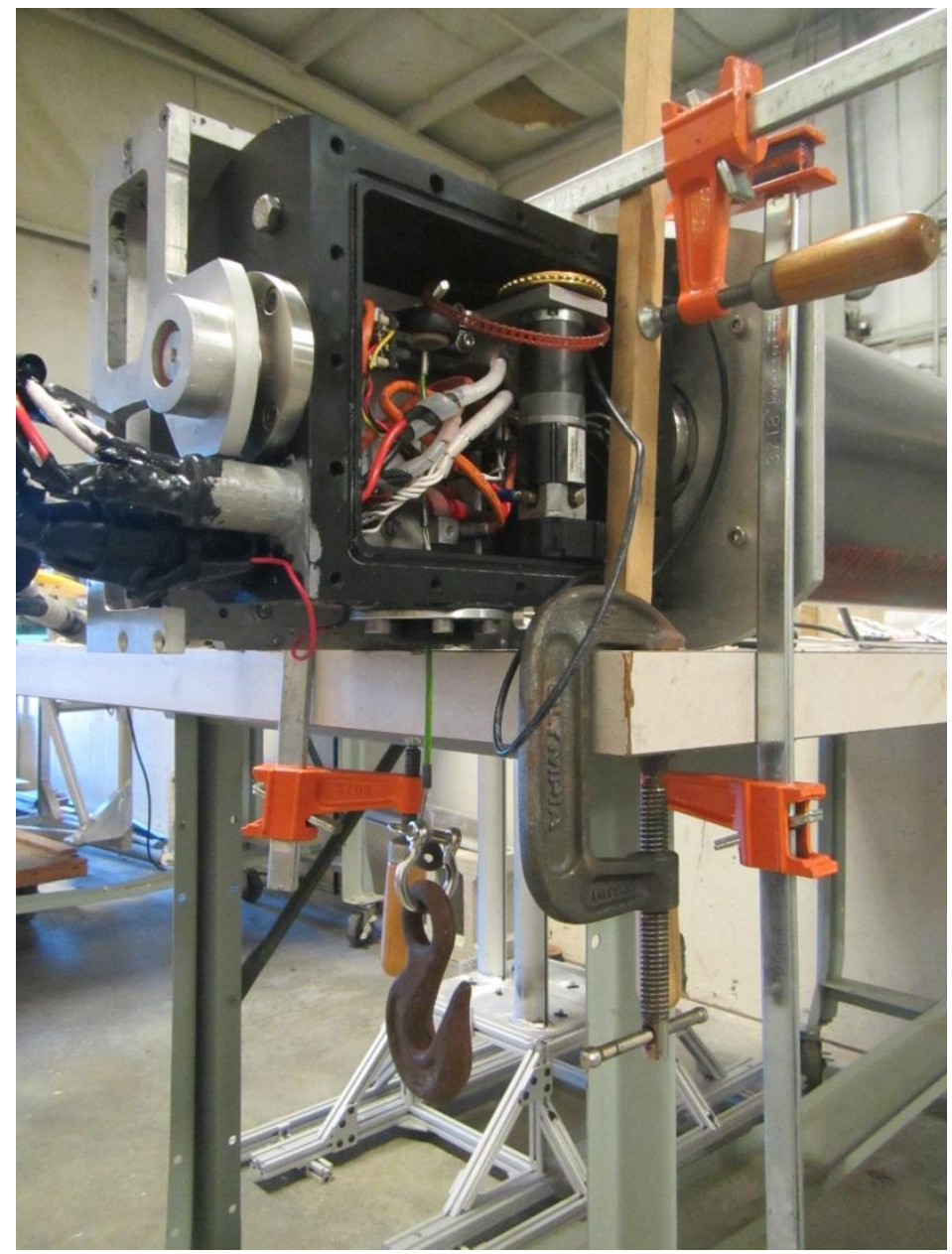

Figure 16. Applying an x-direction force

For $\mathrm{z}$, the weights were hung directly out the top of the delrin housing. 


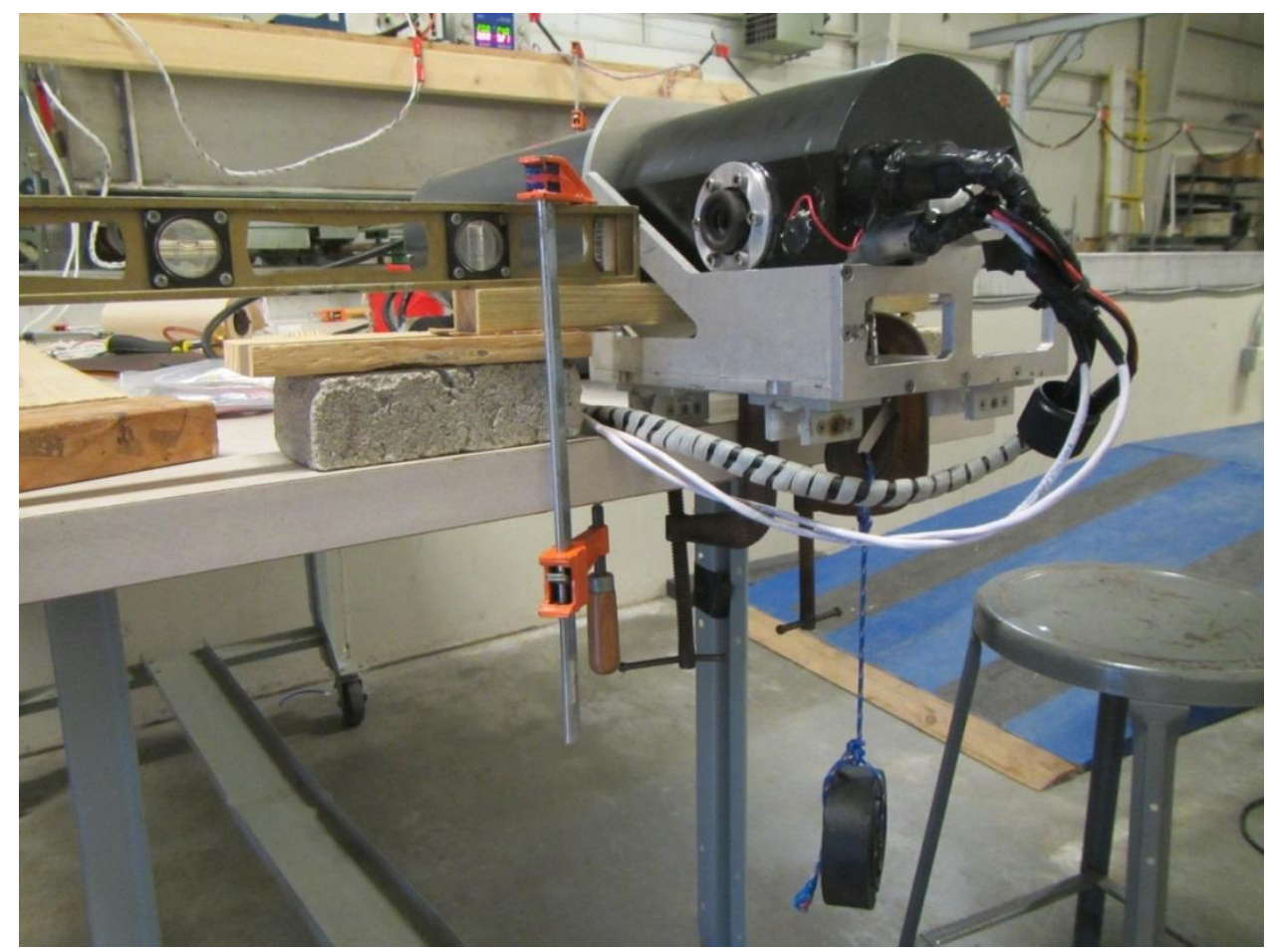

Figure 17. Applying a z-direction force

For $y$, it was more complicated because the delrin housing of the pitch canister would not allow any direct $y$-force to be applied. To obtain calibration for $y$, the dual canister had to be propped up at a $45^{\circ}$ to the horizontal, and the weights were hung obtaining a sensor response which was due to simultaneously applied $\mathrm{y}$ - and z-forces. The procedure to find the responses due to only y-forces is described in following sections. 


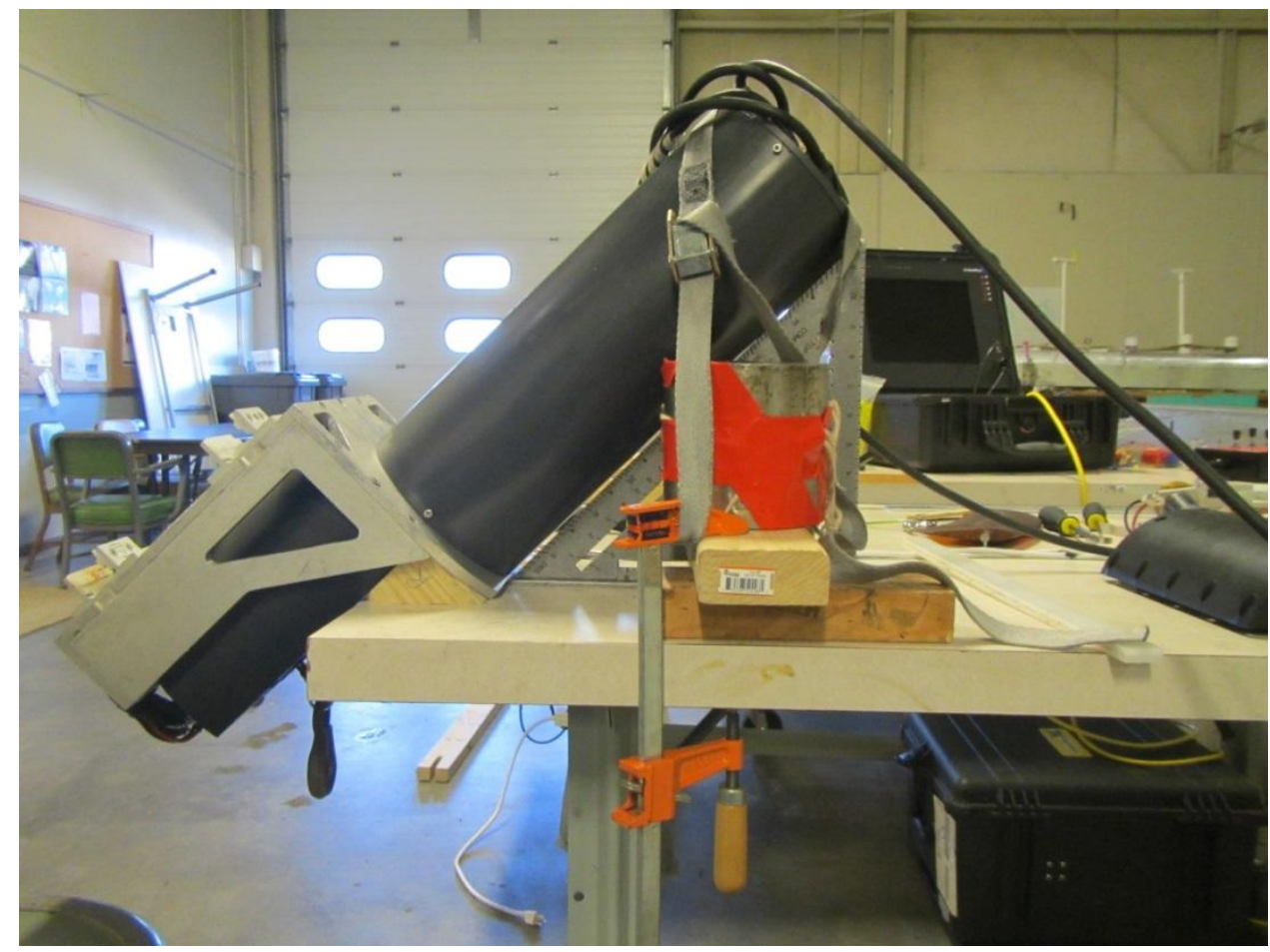

Figure 18. Applying simultaneous $y$ - and z-direction forces

Additionally, the new calibration procedure does not neglect the cross-terms, however small, and their effect is used through all calculations to find lift and thrust forces on the flapping foil. The only disadvantage to directly hanging weights from the bearings is that only one direction of applied force could be used to find the sensitivity matrices for each sensor. For sensor axis $\mathrm{x}$, force could only be applied out the foil hole, thus it was a negative $y$-force in the body coordinate frame. For sensor axis z, force could only be applied out the open delrin top of the pitch canister, thus it was a negative z-force in the body coordinate frame. For sensor axis y, it was in the forward direction out the open delrin top of the pitch canister, thus it was a positive $x$-force in the body coordinate frame.

2.1.5.3. Sensitivity and calibration matrices 
For both the sensitivity and calibration matrices, the following nomenclature is used:

$$
A_{c d}^{b}
$$

Equation 8. Example of nomenclature

"A" can be either "Se" or "Ca" to identify itself as an element in a sensitivity or a calibration matrix, respectively. " $b$ " can either be "1" or " 2 " to identify whether the first or second force sensor is being referred to. "c" can either be " $\mathrm{x}$ ", " $\mathrm{y}$ ", or " $z$ " to identify the direction, in the particular sensor coordinate frame, of the sensor response. Lastly, "d" can be either "x", "y", "z", or "yz" to identify the direction, in the particular sensor coordinate frame, of the applied force. Putting that all together, the following element:

$$
S e_{z x}^{1}
$$

Equation 9. Nomenclature in use

reads as sensor 1 sensitivity in the $\mathrm{z}$-direction as a result of an applied $\mathrm{x}$-force. As stated earlier, the sensitivity matrix elements were obtained from the appropriate slopes. Using the example above in Equation 9, that particular element will be obtained from the figure directly below. In Figure 19, the left chart for sensor 1 has a red linear trend line which corresponds to the sensitivity element in question. Units of sensitivity are Volts/Newtons. 

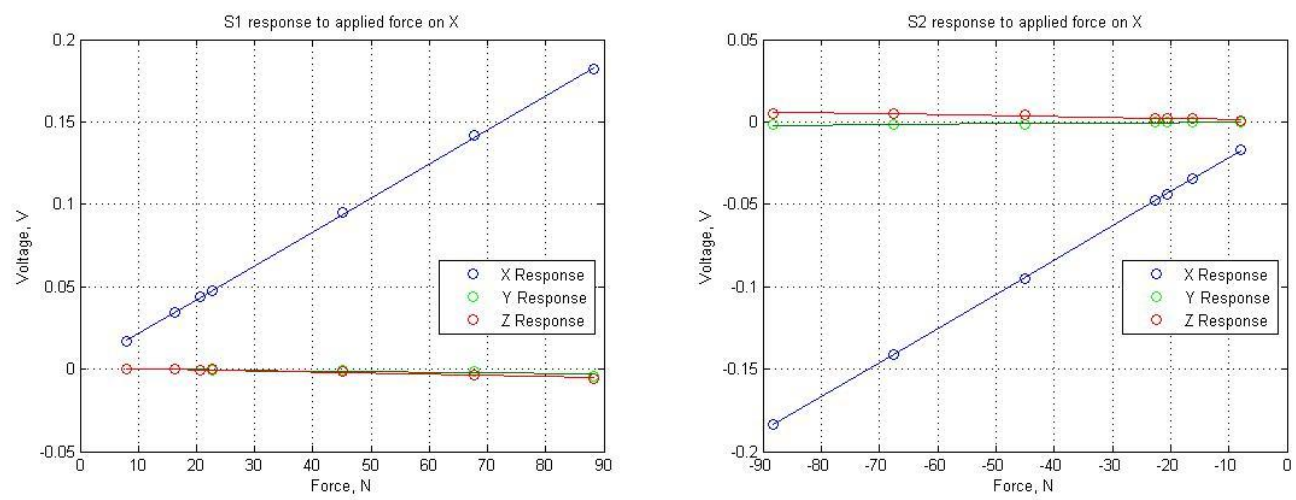

Figure 19. Sensor responses due to applied $x$-force
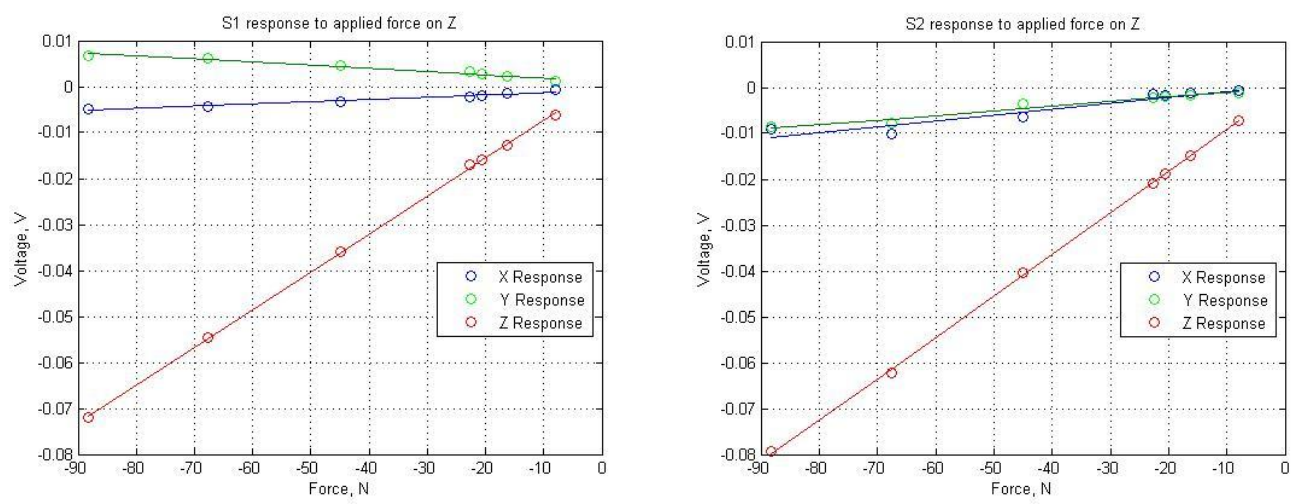

Figure 20. Sensor responses due to applied z-force
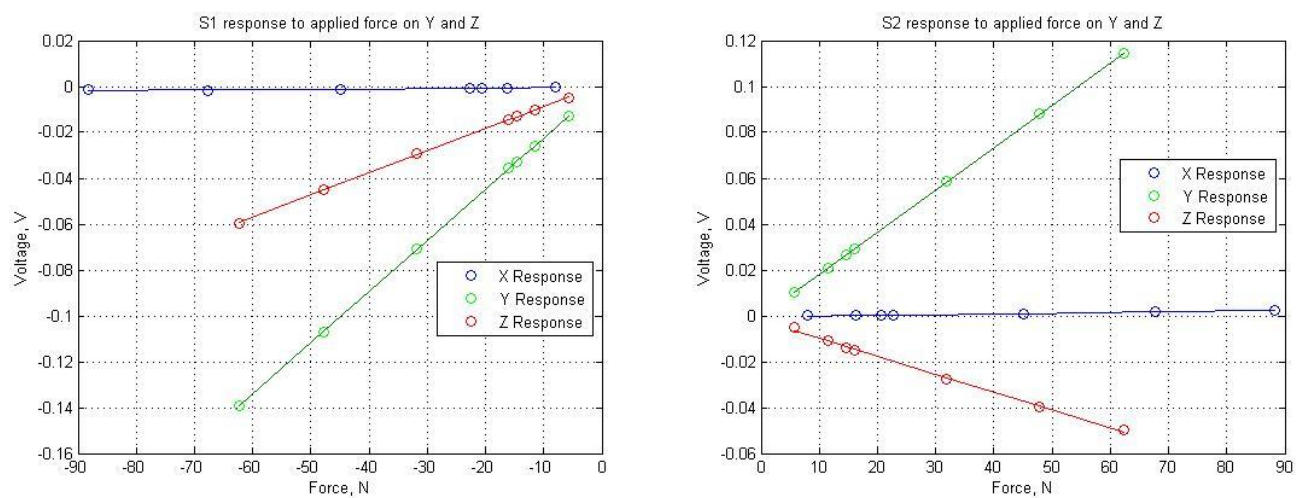

Figure 21. Sensor responses due to applied y-and z-forces

The raw, uncorrected sensitivity matrices were found to be: 


$$
\begin{aligned}
S e_{\text {raw }}^{1} & =\left[\begin{array}{lll}
S e_{x x}^{1} & S e_{x(y z)}^{1} & S e_{x z}^{1} \\
S e_{y x}^{1} & S e_{y(y z)}^{1} & S e_{y z}^{1} \\
S e_{z x}^{1} & S e_{z(y z)}^{1} & S e_{z z}^{1}
\end{array}\right]=\left[\begin{array}{ccc}
0.00206 & 0.0000153 & 0.0000491 \\
-0.0000406 & 0.00223 & -0.0000682 \\
-0.0000694 & 0.000962 & 0.000823
\end{array}\right] \\
S e_{\text {raw }}^{2} & =\left[\begin{array}{lll}
S e_{x x}^{2} & S e_{x(y z)}^{2} & S e_{x z}^{2} \\
S e_{y x}^{2} & S e_{y(y z)}^{2} & S e_{y z}^{2} \\
S e_{z x}^{2} & S e_{z(y z)}^{2} & S e_{z z}^{2}
\end{array}\right]=\left[\begin{array}{ccc}
0.00207 & 0.0000286 & 0.000128 \\
0.0000229 & 0.00184 & 0.000101 \\
-0.0000537 & -0.000780 & 0.000905
\end{array}\right]
\end{aligned}
$$

Equation 10. Raw sensitivity matrices

The middle columns are sensitivities due to the simultaneously applied y- and zforce, as discussed above. For sensor 1, the sensitivity due to only y-applied force was found to be the sensitivity due to applied yz-force minus the sensitivity due to applied z-force. They are subtracted because in the sensor 1 coordinate frame, negative $\mathrm{z}$-force gives a negative y-response.

$$
\left[\begin{array}{c}
S e_{x y}^{1} \\
S e_{y y}^{1} \\
S e_{z y}^{1}
\end{array}\right]=\left[\begin{array}{c}
S e_{x(y z)}^{1} \\
S e_{y(y z)}^{1} \\
S e_{z(y z)}^{1}
\end{array}\right]-\left[\begin{array}{c}
S e_{x z}^{1} \\
S e_{y z}^{1} \\
S e_{z z}^{1}
\end{array}\right]
$$

Equation 11. Sensor 1 correction for applied y-force

Similarly, for sensor 2 the sensitivity due to only y-applied force was found to be the sensitivity due to applied yz-force plus the sensitivity due to applied z-force. This time, in the sensor 2 coordinate frame, negative $\mathrm{z}$-force gives a positive $\mathrm{y}$ response.

$$
\left[\begin{array}{l}
S e_{x y}^{2} \\
S e_{y y}^{2} \\
S e_{z y}^{2}
\end{array}\right]=\left[\begin{array}{c}
S e_{x(y z)}^{2} \\
S e_{y(y z)}^{2} \\
S e_{z(y z)}^{2}
\end{array}\right]+\left[\begin{array}{c}
S e_{x z}^{2} \\
S e_{y z}^{2} \\
S e_{z z}^{2}
\end{array}\right]
$$

Equation 12. Sensor 2 correction for applied $y$-force 
Thus the following has been obtained for the corrected sensitivity matrices.

$$
\begin{gathered}
S e^{1}=\left[\begin{array}{lll}
S e_{x x}^{1} & S e_{x y}^{1} & S e_{x z}^{1} \\
S e_{y x}^{1} & S e_{y y}^{1} & S e_{y z}^{1} \\
S e_{z x}^{1} & S e_{z y}^{1} & S e_{z z}^{1}
\end{array}\right]=\left[\begin{array}{ccc}
0.00206 & -0.0000338 & 0.0000491 \\
-0.0000406 & 0.00230 & -0.0000682 \\
-0.0000694 & 0.000139 & 0.000823
\end{array}\right] \\
S e^{2}=\left[\begin{array}{lll}
S e_{x x}^{2} & S e_{x y}^{2} & S e_{x z}^{2} \\
S e_{y x}^{2} & S e_{y y}^{2} & S e_{y z}^{2} \\
S e_{z x}^{2} & S e_{z y}^{2} & S e_{z z}^{2}
\end{array}\right]=\left[\begin{array}{lll}
0.00207 & 0.000157 & 0.000128 \\
0.0000229 & 0.00194 & 0.000101 \\
-0.0000537 & 0.000125 & 0.000905
\end{array}\right]
\end{gathered}
$$

Equation 13. Final sensitivity matrices

Allowing the following response plots to be produced.
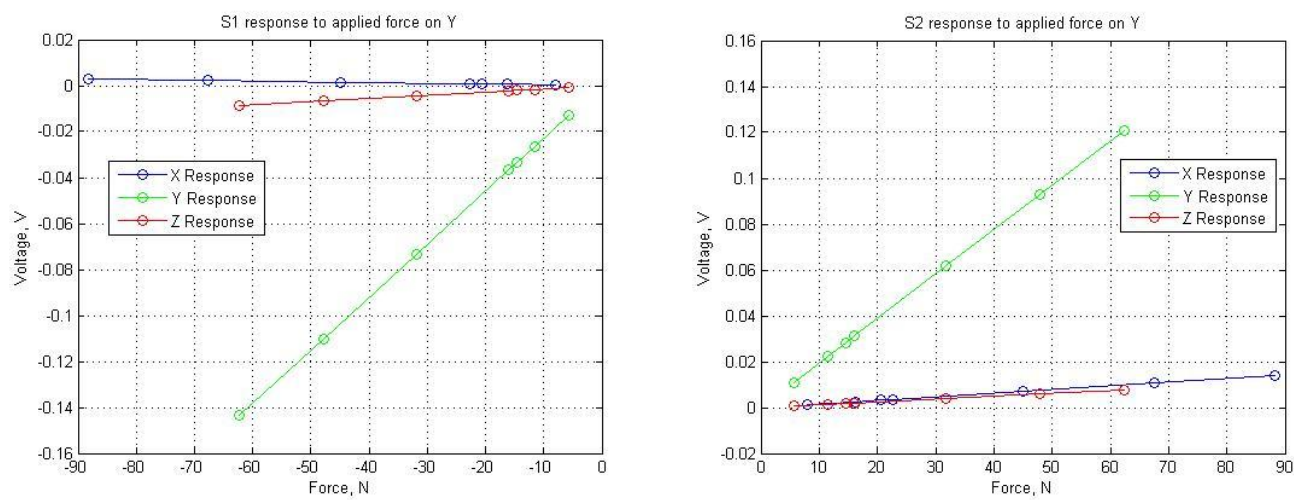

Figure 22. Sensor responses due to applied y-force

What remains now is to obtain the calibration matrices from those sensitivity matrices.

$$
\begin{gathered}
C a^{1}=\operatorname{inv}\left(S e^{1}\right)=\left[\begin{array}{lll}
C a_{x x}^{1} & C a_{x y}^{1} & C a_{x z}^{1} \\
C a_{y x}^{1} & C a_{y y}^{1} & C a_{y z}^{1} \\
C a_{z x}^{1} & C a_{z y}^{1} & C a_{z z}^{1}
\end{array}\right]=\left[\begin{array}{ccc}
484.6 & 8.826 & -28.18 \\
9.719 & 432.8 & 35.28 \\
39.23 & -72.35 & 1207
\end{array}\right] \\
C a^{2}=\operatorname{inv}\left(S e^{2}\right)=\left[\begin{array}{lll}
C a_{x x}^{2} & C a_{x y}^{2} & C a_{x z}^{2} \\
C a_{y x}^{2} & C a_{y y}^{2} & C a_{y z}^{2} \\
C a_{z x}^{2} & C a_{z y}^{2} & C a_{z z}^{2}
\end{array}\right]=\left[\begin{array}{ccc}
481.8 & -34.85 & -64.26 \\
-7.228 & 519.7 & -56.98 \\
29.59 & -73.85 & 1109
\end{array}\right]
\end{gathered}
$$

Equation 14. Calibration matrices 
The calibration matrices are simply the inverse of the sensitivity matrices. Units of calibration are Newtons/Volts. The calibration matrices are used together with the output voltages of each sensor in order to find the output forces experienced at each sensor. The final step is to multiply the calibration matrix by the output voltages:

$$
\left[\begin{array}{lll}
C a_{x x}^{1} & C a_{x y}^{1} & C a_{x z}^{1} \\
C a_{y x}^{1} & C a_{y y}^{1} & C a_{y z}^{1} \\
C a_{z x}^{1} & C a_{z y}^{1} & C a_{z z}^{1}
\end{array}\right]\left[\begin{array}{c}
V_{x}^{1} \\
V_{y}^{1} \\
V_{z}^{1}
\end{array}\right]=\left[\begin{array}{l}
C a_{x x}^{1} V_{x}^{1}+C a_{x y}^{1} V_{y}^{1}+C a_{x z}^{1} V_{z}^{1} \\
C a_{y x}^{1} V_{x}^{1}+C a_{y y}^{1} V_{y}^{1}+C a_{y z}^{1} V_{z}^{1} \\
C a_{z x}^{1} V_{x}^{1}+C a_{z y}^{1} V_{y}^{1}+C a_{z z}^{1} V_{z}^{1}
\end{array}\right]=\left[\begin{array}{c}
F_{x}^{1} \\
F_{y}^{1} \\
F_{z}^{1}
\end{array}\right]
$$

Equation 15. Obtaining forces from calibration matrices and sensor output voltages

Thus each force output in a given direction contains contributions from all three directions, since there is cross-sensitivity seen in the sensors. The equation above is listed for sensor 1 and the same equation is used for sensor 2 . Note the output force directions are in the individual sensor reference frame, the directions were shown above in Figure 15. This means that there will still be a transformation required from sensor frame forces to get body frame forces. The transformation is described later.

Although the method of calibration was improved in this work, errors may still have been introduced. The force sensors require in situ calibration once installed, since they do not arrive calibrated from the manufacturer. The new calibration method directly applies force to each bearing, attached above each force sensor. To accomplish that though, the pitch shaft had to be removed. To re-install the pitch shaft, the bearings needed to be unscrewed from their mounting plates above the force sensors, slipped over the pitch shaft, and then placed back into the delrin housing. Errors were possibly introduced due to a 
change in installation after calibration. Additionally, only one direction of each coordinate axis could be calibrated, since the physical arrangement of the pitch canister did not allow force to be applied in the opposite direction. The assumption was that the sensor response was linear in the opposite direction.

\subsection{Experimental Method}

\subsubsection{Experimental Setup}

The large tow tank in the Sheets Laboratory on the Bay Campus was used for this work, the most important aspect of which was to configure the 'beach'. A wave generator (not used for this work) is installed at the beginning of this $30 \mathrm{~m}$ tow tank, and the floor of the tow tank is called a beach because it is often configured as a sloping beach, to observe how waves run up or break. The tow tank is actually a rectangular prism, cast in concrete, and the beach is comprised of metal plates. Each metal plate is $2.5 \mathrm{~m}$ long and there are seven plates at the end of the tow tank. The metal plates are configured by: attaching winches to the sides of the tank, clipping the winch strap end into the seam between each metal plate, tightening the winch strap as a pair to ensure uniformity, unscrewing the large stainless steel bolts that pin the metal plate seams into the inside concrete wall of the tow tank, using the winch to raise or lower the seam as appropriate, screwing the stainless steel bolts back in to pin the metal plate, and then loosening and removing the winch strap. 


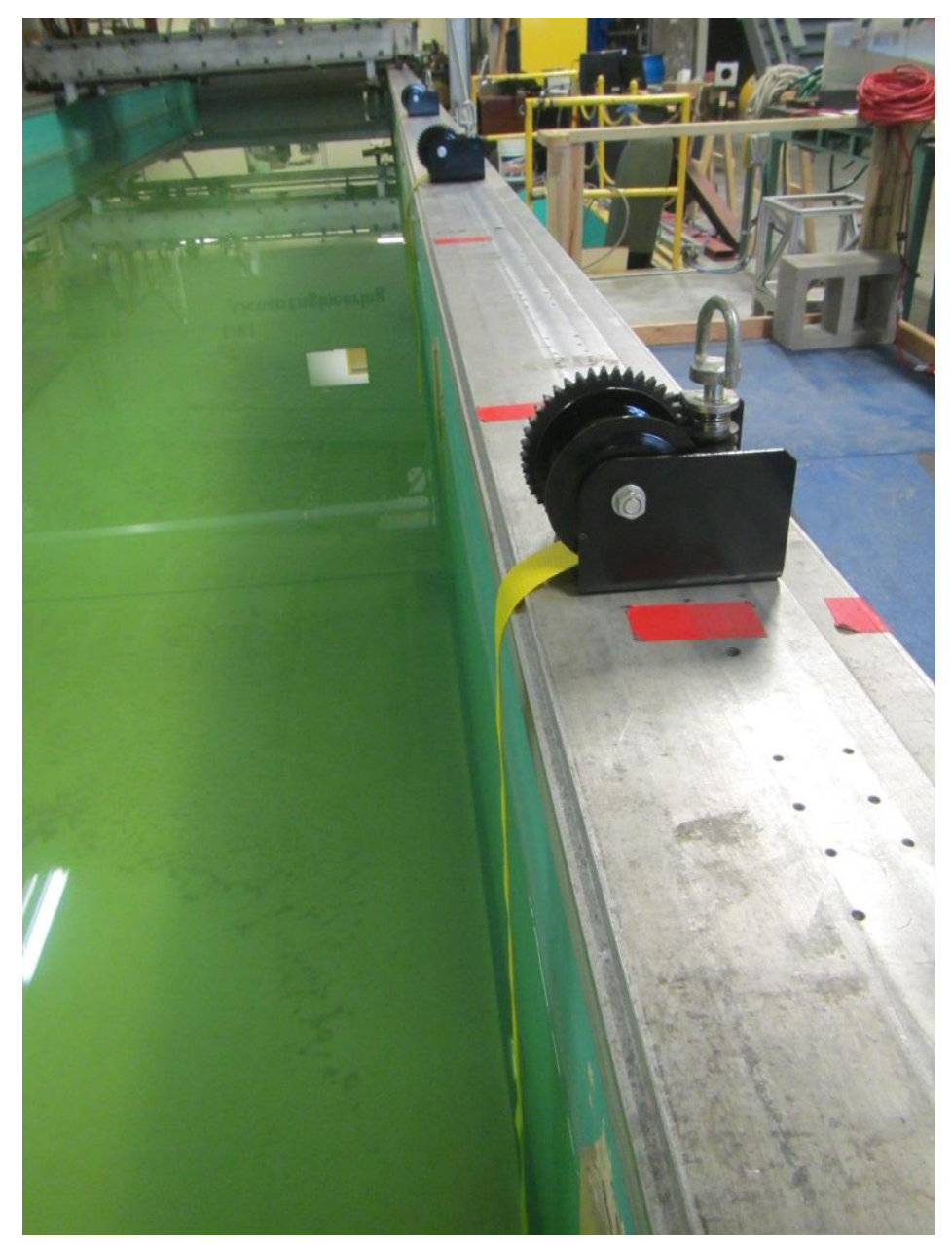

Figure 23. Winches ready to move the metal plate seams of the beach

The tow tank was configured with the profile of water depths as depicted below:

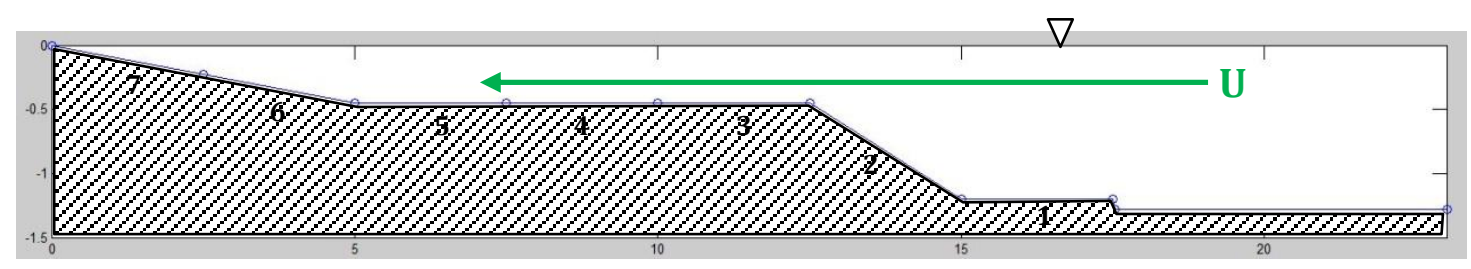

Figure 24. Representation of tow tank water depth

This gave a deep portion of $8 \mathrm{~m}$ of run (tank bottom and plate 1), a transition portion of $2.5 \mathrm{~m}$ (plate 2), and then a shallow portion of $7 \mathrm{~m}$ of run (plates 3,4 , and 5). Because the carriage was run from deep to shallow, in one continuous run the 
flapping foil was able to experience flapping in the middle of the water column (hereafter referred to as "freestream") and flapping very close to the beach ("near bottom"). For the freestream case at the roll axis the H/c=8.0, indeed, the water depth was so deep relative to the foil chord that if the foil was rotated to point straight down toward the beach, the tip would still be at $\mathrm{H} / \mathrm{c}=2.8$. Thus for the freestream case, there is absolutely no influence from the ground, nor from the walls of the tow tank. In the freestream case, the foil flaps free and clear in deep water. When the foil is at a roll amplitude of zero, it is $0.32 \mathrm{~m}$ below the water free surface (an $\mathrm{H} / \mathrm{c}$ equivalent of 3.2 below water).

In order to get the largest magnitude response for ground effect, the desire was to have the dual canister move as close to the beach as possible. Due to the radius of the dual canister itself and the metal support bracket, it is only possible to get the roll axis in ground effect to a minimum $\mathrm{H} / \mathrm{c}=1.1$, that is a physical limitation. It is a realistic limitation however, since the same and even larger distances away from the ground would be encountered on deployed UUVs that use flapping foils for propulsion, due to the associated mechanisms necessary for roll and pitch. Using a combination of the fixed length carriage attachment structure and wooden blocks to shore it up on the tow tank carriage itself, the metal support bracket of the dual canister was able to be placed $1 \mathrm{~cm}$ away from the ground. Note that the overall length of the carriage attachment with dual canister attached was $1.38 \mathrm{~m}$, and its weight was in excess of approximately $350 \mathrm{~N}$. Note the following photograph, showing a front view of the dual canister at the closest configuration to the ground (looking in the body 
frame system in negative $\mathrm{x}$-direction). The fin tip is touching the ground, at a roll amplitude of a little over $13^{\circ}$.

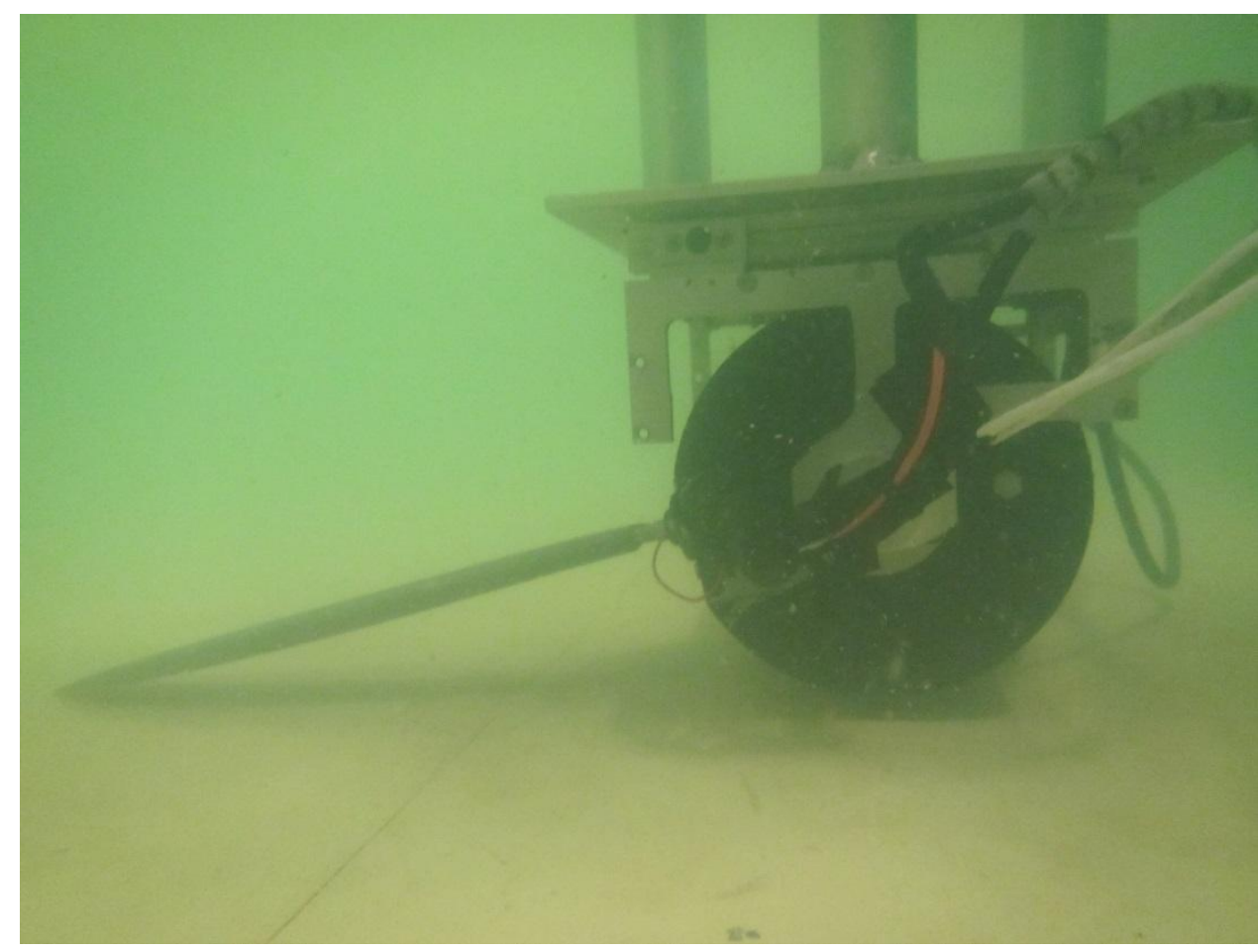

Figure 25. Dual canister closest to the beach

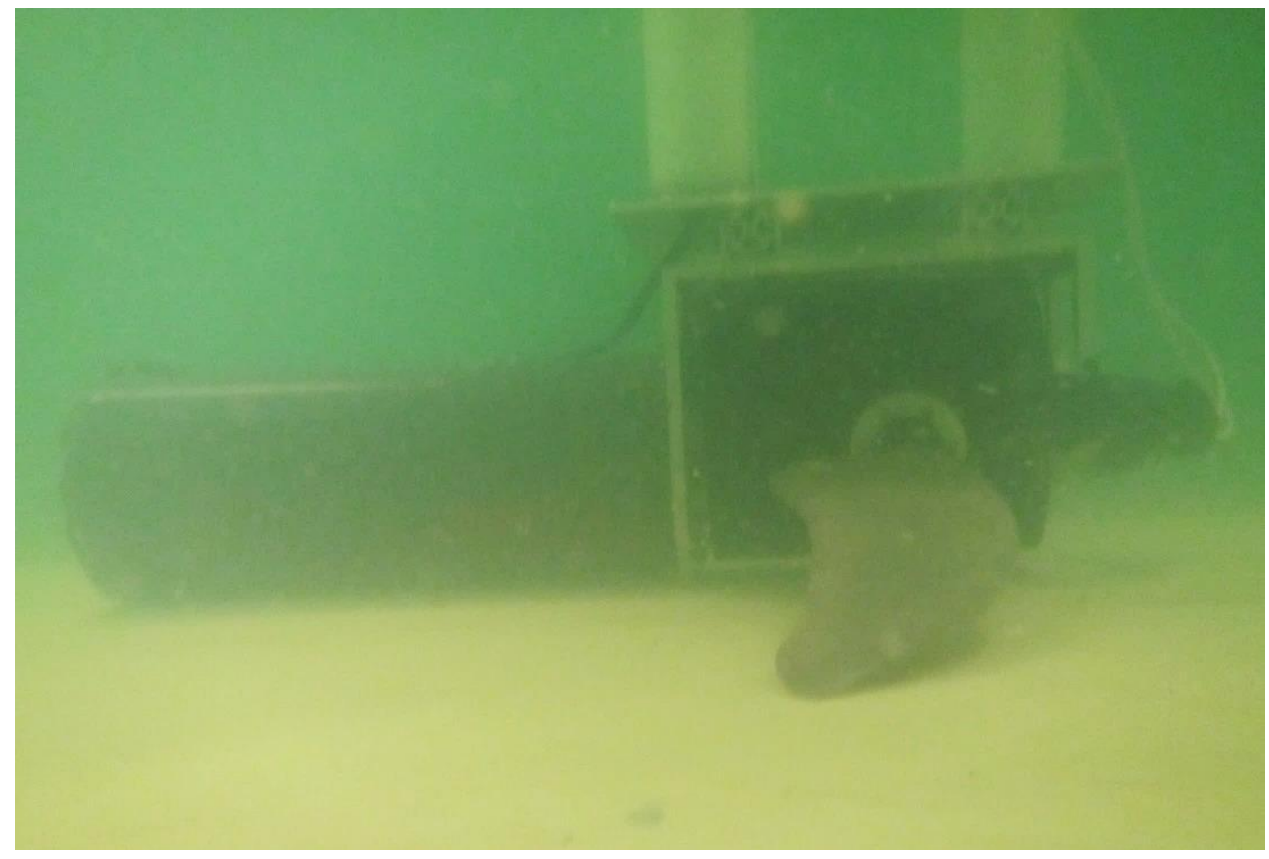

Figure 26. Side view of dual canister near bottom 
The maximum roll amplitude can only be $12^{\circ}$, and that value has been used for all experiments in this work. Care was taken to ensure that the bottom of the dual canister would not impact the beach. The most important aspect was the safety and security of school assets and equipment. To establish the height of wooden blocks needed in order to get the foil as close as possible to the beach, the carriage was operated by one individual outside the tow tank. The author was submerged in the tow tank, next to the dual canister, while the carriage went along at a snail's pace. Height of blocks and final metal plate configuration in the tank was determined such that the dual canister will never impact the beach, as long as the carriage is stopped above metal plate 5 . Foil roll amplitude was limited so that only fluid forces over the foil were measured and not forces due to the fin tip impacting the beach. With roll amplitude fixed, the heave to chord ratio is also therefore fixed, found to be $h_{0.7} / c=0.91$ for all experiments.

\subsubsection{Experimental Procedure}

The procedure for data collection runs was identical to the procedure presented in (Rauworth, 2014), with one small change. In this work, the force sensors were powered down and back up again prior to each data run, using the switch on the force sensor collection software.

\subsubsection{Data Processing}

Many of the same data processing techniques from (Rauworth, 2014) were applied in this work. As described above, the results from calibration produce calibration matrices which are multiplied by sensor voltages to give forces experienced at each sensor. Only once sensor forces have been found are 
they translated into the body frame coordinate system. Noting all respective directions from Figure 15, it is easy to see how the following translation is made from sensor forces to body forces, for each respective sensor:

$$
F_{b o d y}^{1}=\left[\begin{array}{l}
F_{x} \\
F_{y} \\
F_{z}
\end{array}\right]_{\text {body }}^{1}=\left[\begin{array}{c}
-F_{y} \\
-F_{x} \\
F_{z}
\end{array}\right]_{\text {sensor }}^{1} \quad \text { and, } \quad F_{\text {body }}^{2}=\left[\begin{array}{c}
F_{x} \\
F_{y} \\
F_{z}
\end{array}\right]_{\text {body }}^{2}=\left[\begin{array}{c}
F_{y} \\
F_{x} \\
F_{z}
\end{array}\right]_{\text {sensor }}^{2}
$$

Equation 16. Translation from sensor frame to body frame

The flapping foil generates lift and thrust forces as it flaps. The lift force is taken to act through the assumed hydrodynamic center, some distance away from the origin. That distance is actually $\mathrm{r}_{0.7}$, given in Equation 2. Lift applies a moment about the body x-axis. That moment is known, because the force sensors have recorded the body forces, and each sensor is a known distance away from the origin. Thus the trail to find lift force at the foil is as follows: record forces encountered at sensor, find the moment at the origin that would produce those forces, divide out $\mathrm{r}_{0.7}$, and finally obtain lift force at the hydrodynamic center. The same procedure is used to find the thrust force developed at the foil assumed hydrodynamic center, which will produce a moment about the body z-axis. The following representation has not been drawn to scale. It shows forces and dimensions related to the pitch canister only illustrating how moments can be calculated to the origin, and then the lift and thrust forces can be backed out. The number superscripts refer to either sensor 1 , or sensor 2 , depending on which corresponding number is used. 


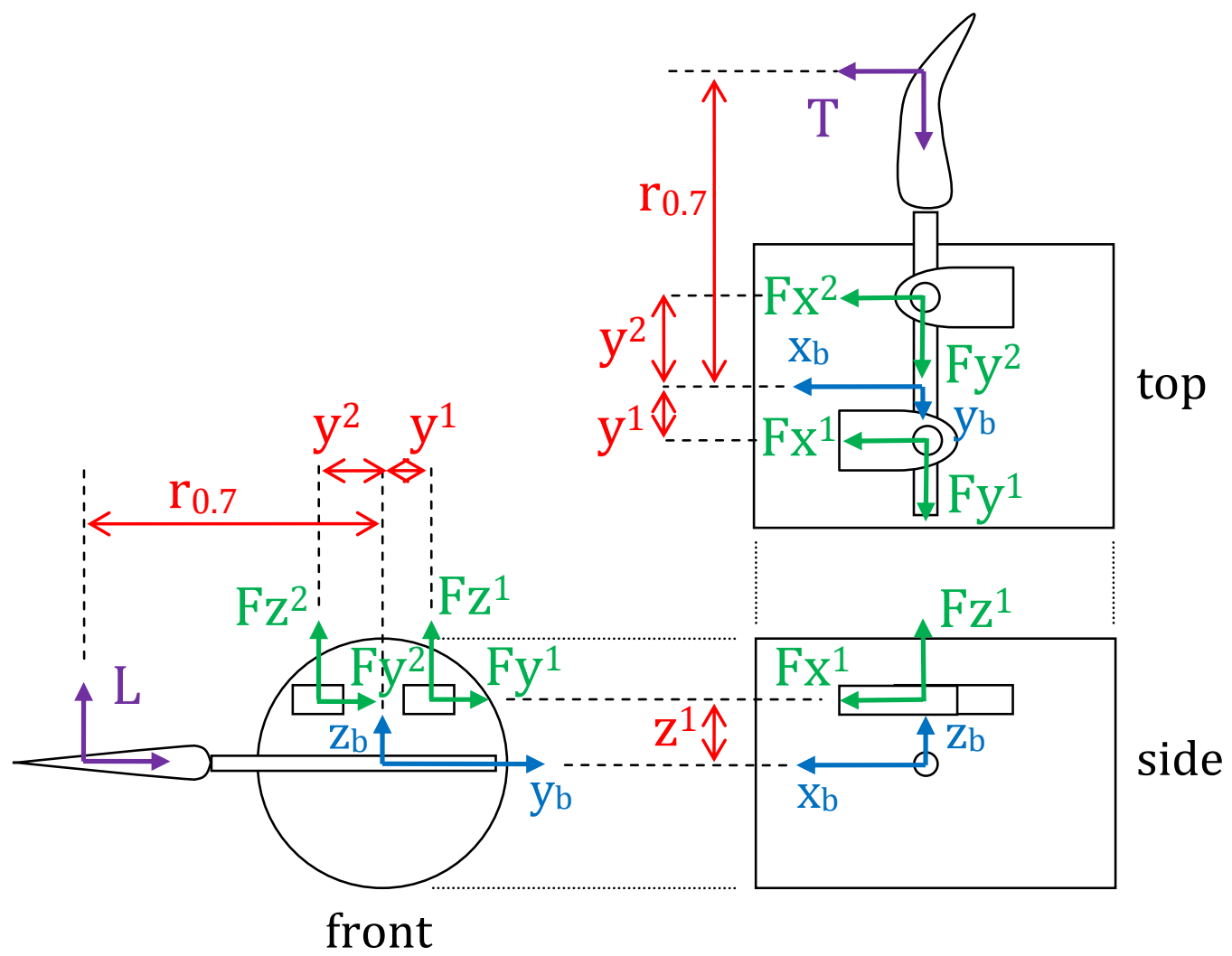

Figure 27. Three view drawing to represent pitch canister

From that figure, it is easy to write out the calculations which must be performed to find lift and thrust forces:

$$
\begin{gathered}
L=\frac{F_{y}^{1}\left(-z^{1}\right)+F_{y}^{2}\left(-z^{2}\right)+F_{z}^{1}\left(y^{1}\right)+F_{z}^{2}\left(-y^{2}\right)}{-r_{0.7}} \\
T=\frac{F_{x}^{1}\left(-y^{1}\right)+F_{x}^{2}\left(y^{2}\right)}{r_{0.7}}
\end{gathered}
$$

Equation 17. Equations to find lift and thrust forces

At this point it is important to mention that the largest assumption, and hence potential source of error in this work, was in the assumed location of the 
hydrodynamic center of the foil. The same location, $\mathrm{r}_{0.7}$ has been consistently used in all the preceding works that have been built upon here, such as in (Polidoro, 2003), (Techet, 2008), and (Rauworth, 2014). The location of the actual hydrodynamic center was still not able to be accurately determined, due to the low resolution and high noise still present in the system. Looking at the equations above it is easy to see that lift and thrust forces calculated can change greatly due to the denominator. The maximum forces that might be calculated would be found if the denominator used was the radius at the root of the foil, similarly, the forces would be minimum if the radius at the tip of the foil was used. That fact provides two bounds, that the lift and thrust forces are to be found in between.

As mentioned before, one run down the tow tank consists of three distinct flapping regimes; namely freestream, transition zone, and near bottom. It was very important to synchronize the LDM readout with the data collected from the force sensors for the next step in data processing. Once the lift and thrust forces are known for the entire run they were phase averaged in order to produce plots that showed the average forces over one flapping cycle. Phase averaging was conducted twice for each run, first for flapping in the freestream, and second for flapping near bottom. The processing code also calculated the maximum values and minimum values of lift and thrust force, as well as the mean lift and mean thrust coefficients for each run, for both freestream and near bottom. All of these values will be used in the next chapter to quantify the ground effect that was observed. 


\section{Results and Discussion}

\subsection{Establishing zero mean lift coefficient}

Prior to collecting data to process, a set of runs were conducted in order to determine the pitch bias that would give a zero mean lift coefficient. The zeroing method for this flapping foil has always proven problematic because the setup inside the pitch canister is not perfect and allows for a few degrees of pitch change. The belt that drives the foil to pitch has been installed as tightly as possible, nonetheless, when the dual canister is powered up and supposedly locked in position it is still possible to rotate the foil in pitch by approximately a degree. A set of 11 runs were conducted for $\theta_{\text {bias }}=-10^{\circ}: 2^{\circ}: 10^{\circ}$ at $\mathrm{St}=0.5$ and $\alpha_{\max }=30^{\circ}$. The goal was to determine the pitch bias for which the flapping foil would exhibit zero mean lift coefficient during the near bottom portion of the run. To the nearest integer it was found to be $3^{\circ}$ pitch bias and was set as the new zero pitch bias:

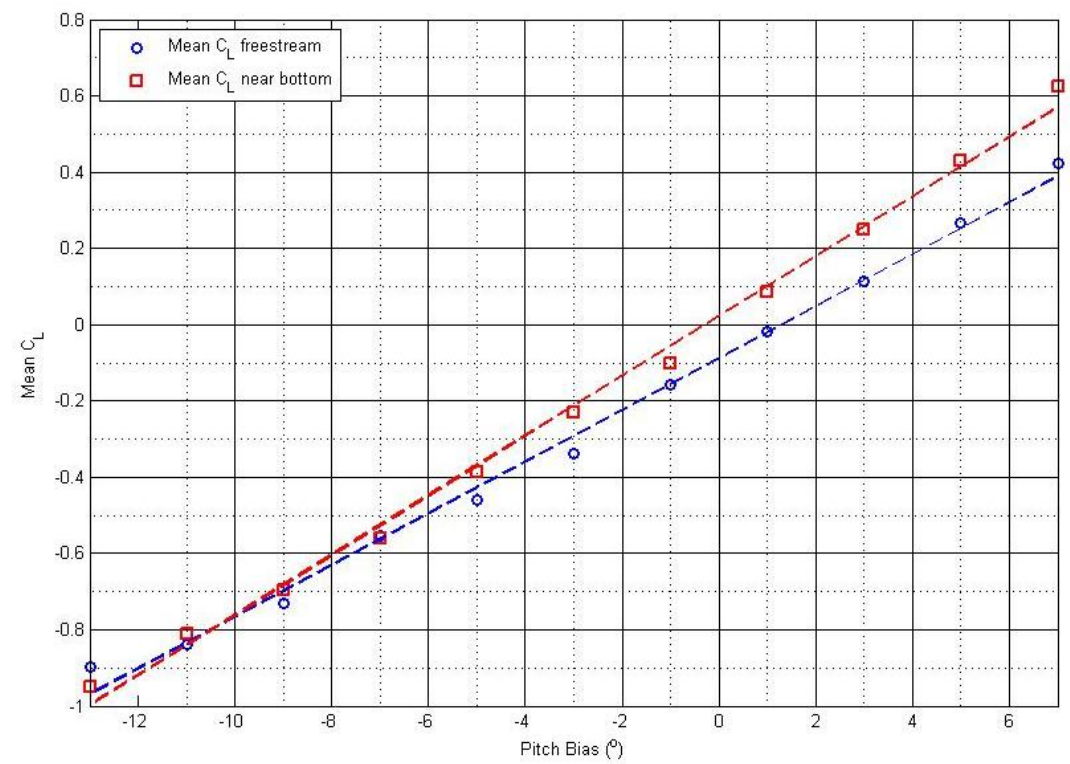

Figure 28. Mean lift coefficient as a function of pitch bias 
Note that to find the pitch bias at which the mean lift coefficient was zero, only one combination of St and $\alpha_{\max }$ was used. This means that the pitch bias was accurate only for that kinematic condition. This may have introduced some error in that a $3^{\circ}$ pitch bias was used as zero, to represent all data points when flapping near bottom. It would have been prohibitive in terms of time, though, to run through a pitch bias sweep for each of the combinations of St and $\alpha_{\max }$ to find a zero mean lift coefficient pitch bias for each. Additionally, the nearest integer pitch bias was used, but it should have been to a fraction of a degree. A positive pitch bias is defined as the pitch amplitude centerline being above the horizontal at the leading edge, so that at zero pitch amplitude the foil has some positive angle of attack. This also means that the pitch centerline at the trailing edge is pointed toward the ground at a positive pitch bias. Figure 28 above shows the variation in mean lift coefficient with close approach to ground. At zero pitch bias, flapping near bottom has a slightly positive lift coefficient, $\mathrm{C}_{\mathrm{L}}=0.022$, whereas flapping in the freestream has a negative lift coefficient, $C_{L}=-0.087$. The result in Figure 28 agrees with the observation in (Licht \& Dahl, 2013) that the freestream case must have a larger than $1^{\circ}$ positive pitch bias in order to generate the same lift as the near bottom case. This effect magnifies as larger mean lift coefficients are sought, such that at a mean $C_{L}=0.4$, the difference is a $2^{\circ}$ positive pitch bias.

\subsection{Contour plots}

The experimental matrix of tests was conducted immediately after finding the pitch bias zero, without changing the roll or pitch position of the dual 
canister. Each run was conducted at the pitch bias value mentioned above, which would nominally give zero mean lift coefficient for the near bottom portion. For reference the following is a table listing the flapping frequency, roll amplitude (AMX), pitch amplitude (AMY), and corresponding Strouhal number and maximum angle of attack. The row that is bold is the test condition at which the zero mean lift coefficient test above was conducted at.

\begin{tabular}{|c|c|c|c|c|c|}
\hline Test No & $\mathrm{f}(\mathrm{Hz})$ & $\mathrm{AMX}\left({ }^{\circ}\right)$ & $\mathrm{AMY}\left({ }^{\circ}\right)$ & $\mathrm{St}$ & $\alpha_{\max }\left({ }^{\circ}\right)$ \\
\hline 1 & 0.82 & 12 & 23 & 0.3 & 20 \\
\hline 2 & 0.82 & 12 & 18 & 0.3 & 25 \\
\hline 3 & 0.82 & 12 & 13 & 0.3 & 30 \\
\hline 4 & 0.82 & 12 & 8 & 0.3 & 35 \\
\hline 5 & 1.1 & 12 & 32 & 0.4 & 20 \\
\hline 6 & 1.1 & 12 & 27 & 0.4 & 25 \\
\hline 7 & 1.1 & 12 & 22 & 0.4 & 30 \\
\hline 8 & 1.1 & 12 & 17 & 0.4 & 35 \\
\hline 9 & 1.37 & 12 & 39 & 0.5 & 20 \\
\hline 10 & 1.37 & 12 & 33 & 0.5 & 25 \\
\hline $\mathbf{1 1}$ & $\mathbf{1 . 3 7}$ & $\mathbf{1 2}$ & $\mathbf{2 8}$ & $\mathbf{0 . 5}$ & $\mathbf{3 0}$ \\
\hline 12 & 1.37 & 12 & 22 & 0.5 & 35 \\
\hline 13 & 1.65 & 12 & 47 & 0.6 & 20 \\
\hline 14 & 1.65 & 12 & 40 & 0.6 & 25 \\
\hline 15 & 1.65 & 12 & 33 & 0.6 & 30 \\
\hline 16 & 1.65 & 12 & 27 & 0.6 & 35 \\
\hline
\end{tabular}

Table 1. Experimental matrix

Having conducted all of the runs listed above, the following contour plots were produced. 


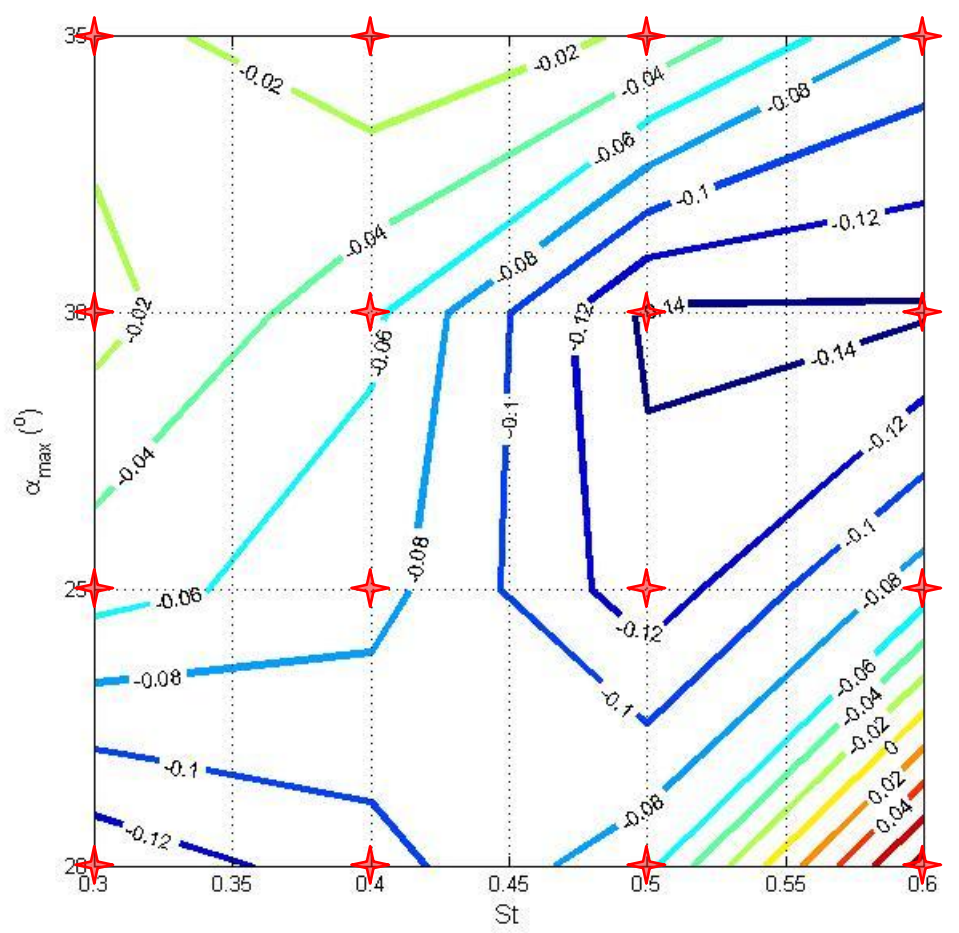

Figure 29. Contour plot for mean lift coefficient in freestream $(\mathrm{H} / \mathrm{c}=8.0)$

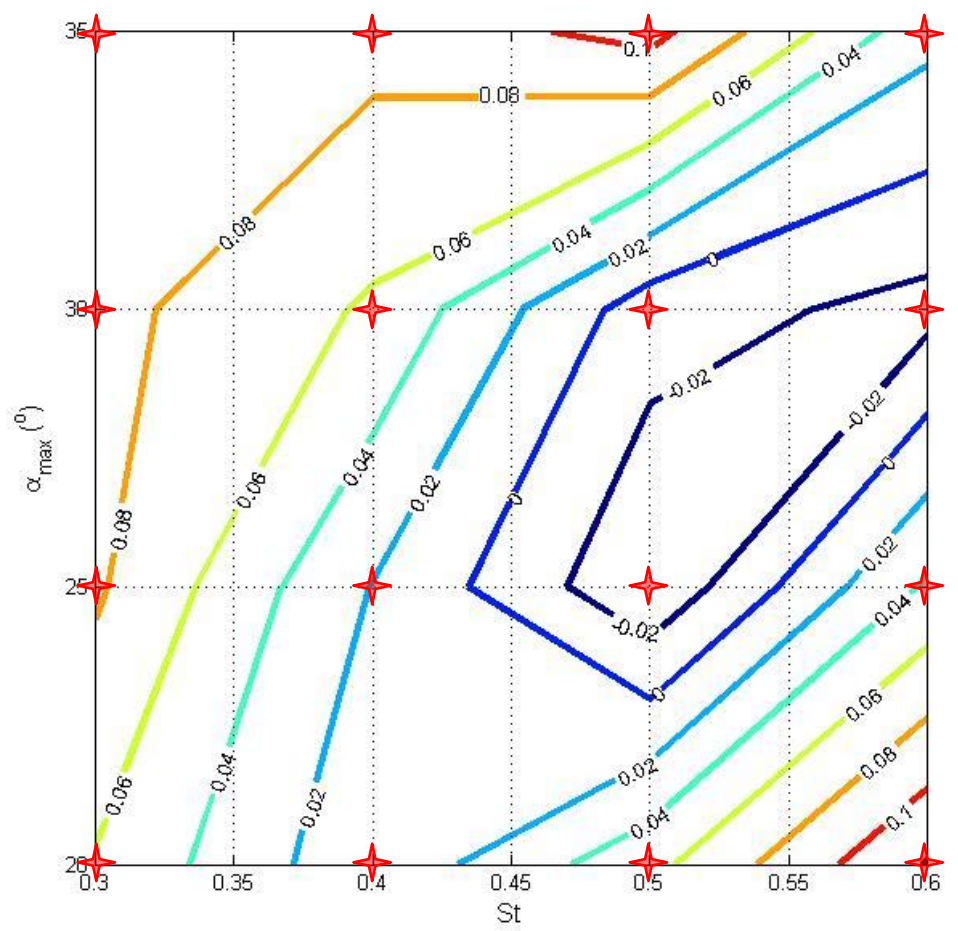

Figure 30. Contour plot for mean lift coefficient near bottom $(H / c=1.1)$ 


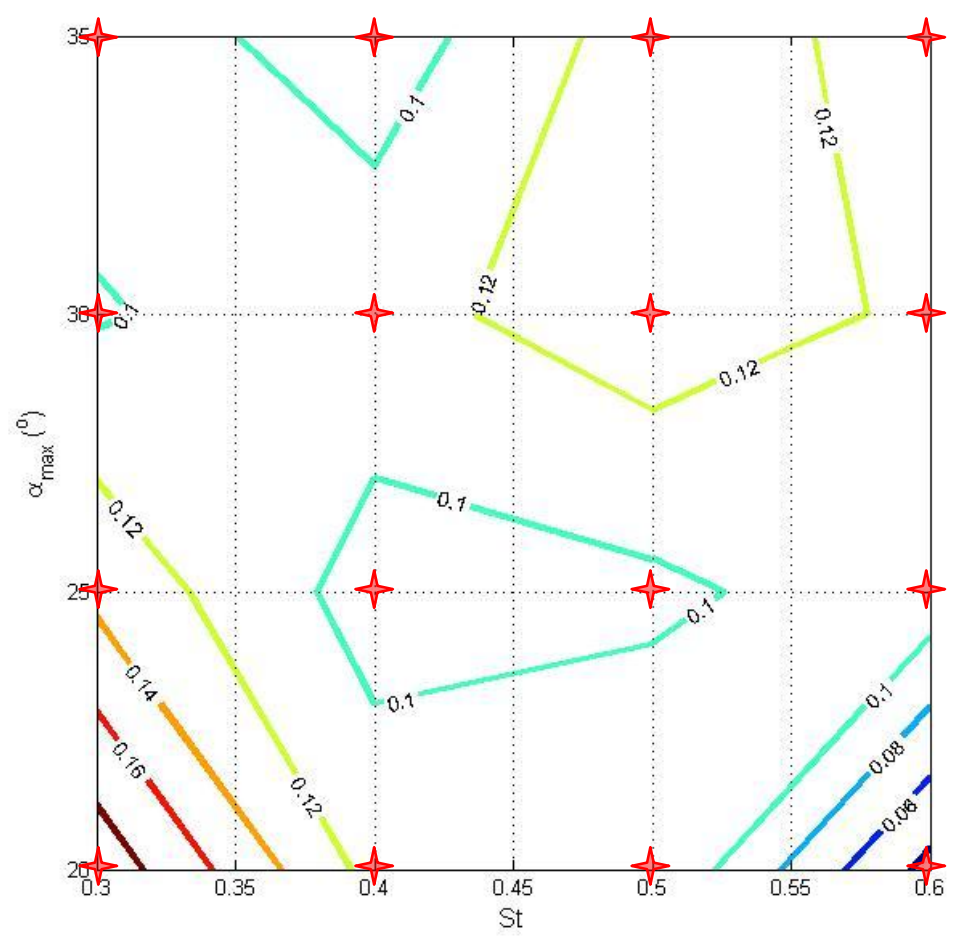

Figure 31. Contour plot for change in mean lift coefficient

Notice immediately that in Figure 29 almost every data point is a negative mean lift coefficient, again highlighting that in the freestream, there will be a negative lift force generated. The only region for which flapping in the freestream generates positive lift is for the highest St and lowest $\alpha_{\max }$, which indicates most aggressive flapping motion, having the largest frequency and the largest pitch amplitude. In Figure 30, the opposite is true, meaning that there is only a small region of three data points where the mean lift coefficient is negative. Perhaps most helpful is to look at Figure 31Figure 31, which shows the difference between flapping near bottom and in the freestream. The data is plotted for $C_{\mathrm{L}}$ near bottom minus $\mathrm{C}_{\mathrm{L}}$ in freestream. It is clear that at every point in the test matrix the mean lift coefficient near the bottom is higher than the mean lift coefficient in 
the freestream. This indicates that the foil is in ground effect for every case tested. The largest change in mean lift coefficient is for $\mathrm{St}=0.3$ and $\alpha_{\max }=20^{\circ}$, and then for a region where $\mathrm{St}=0.5$ and $\alpha_{\max }=30^{\circ}$ and $35^{\circ}$. The smallest change in mean lift coefficient is to be found for $\mathrm{St}=0.6$ and $\alpha_{\max }=20^{\circ}$. Notice that for a given $\alpha_{\max }=20^{\circ}$, the largest and smallest mean lift coefficient changes are found. For the case of low St (low frequency), each flap is long in duration, producing a short but broad lift trace as a function of time. For the case of high St (high frequency), each flap is short in duration, producing a taller but narrower lift trace as a function of time. Because low St equates to larger change in mean lift coefficient than high St, the value for mean lift must be larger for low St, due to that longer duration flap.

The boost in lift due to ground effect has been revealed, so now to determine if there is a benefit to the thrust produced. Based on aerospace engineering, the expectation is there will be some benefit, as induced drag is decreased in ground effect. However, the study by (Blevins \& Lauder, 2013) showed that there is not necessarily a locomotor advantage. Thrust force produced by the foil on the dual canister has been defined as positive forward, namely positive in the body $\mathrm{x}$-direction. 


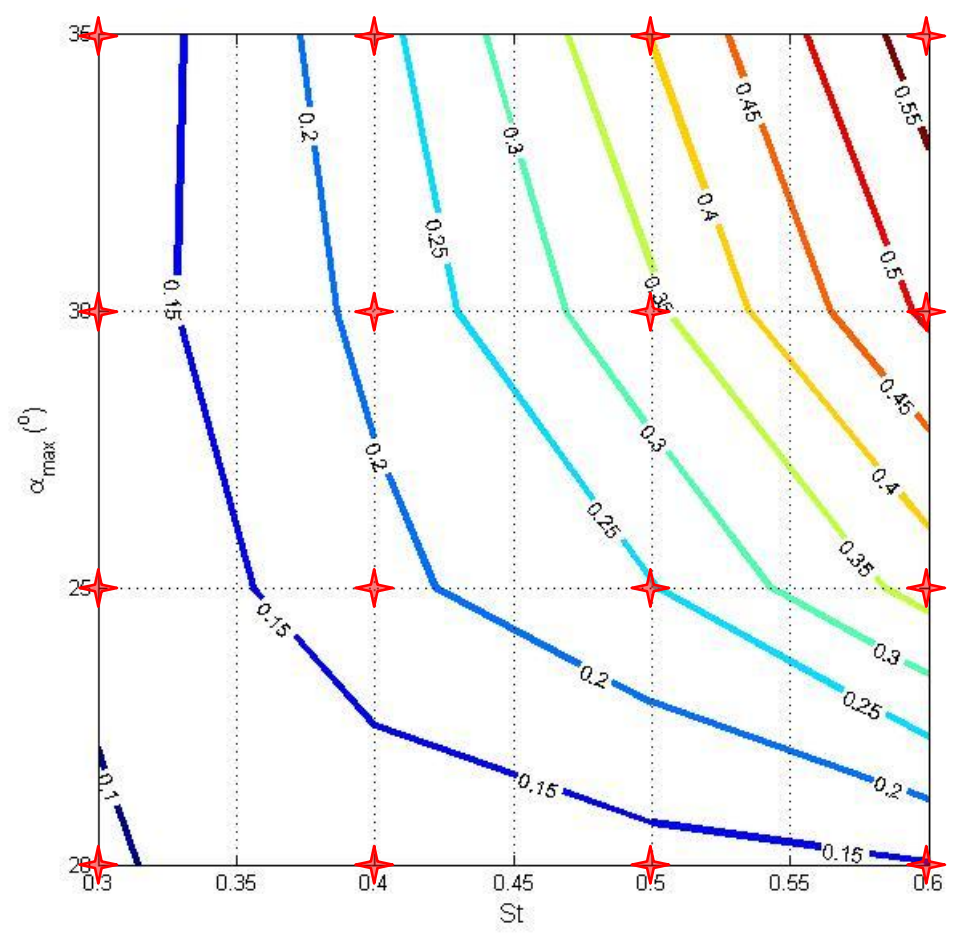

Figure 32. Contour plot for mean thrust coefficient in freestream $(H / c=8.0)$

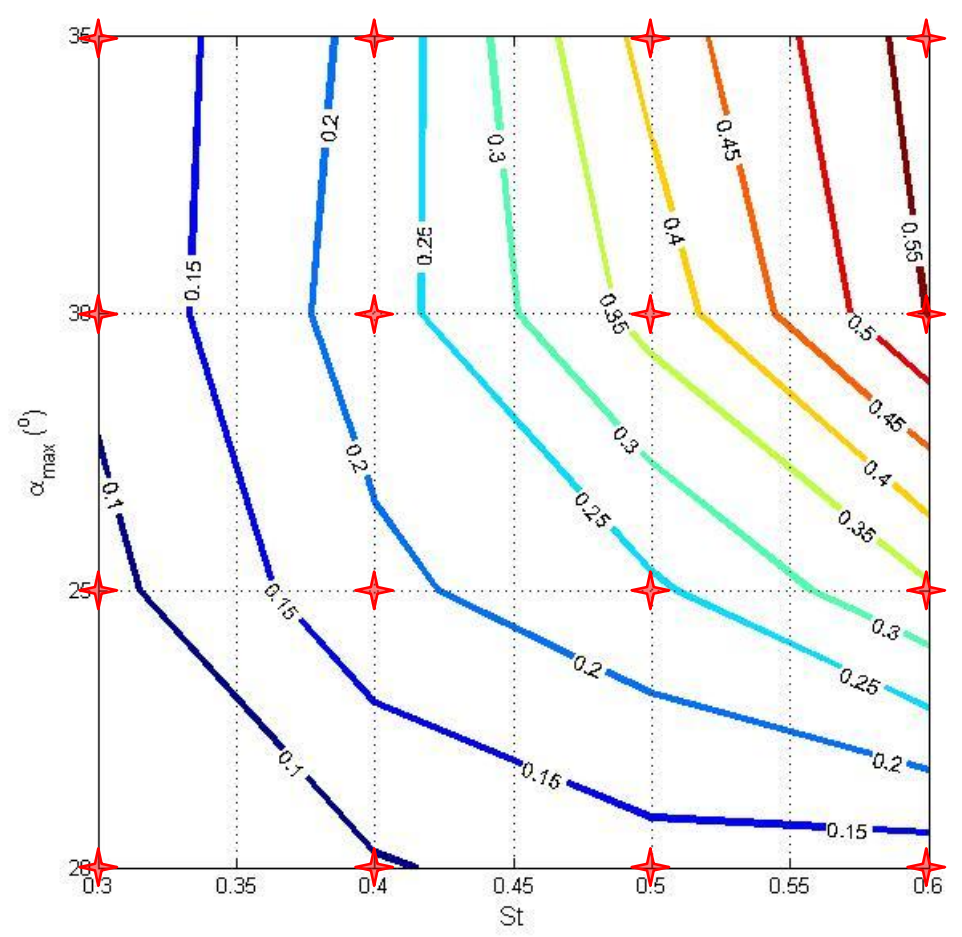

Figure 33. Contour plot for mean thrust coefficient near bottom $(H / c=1.1)$ 


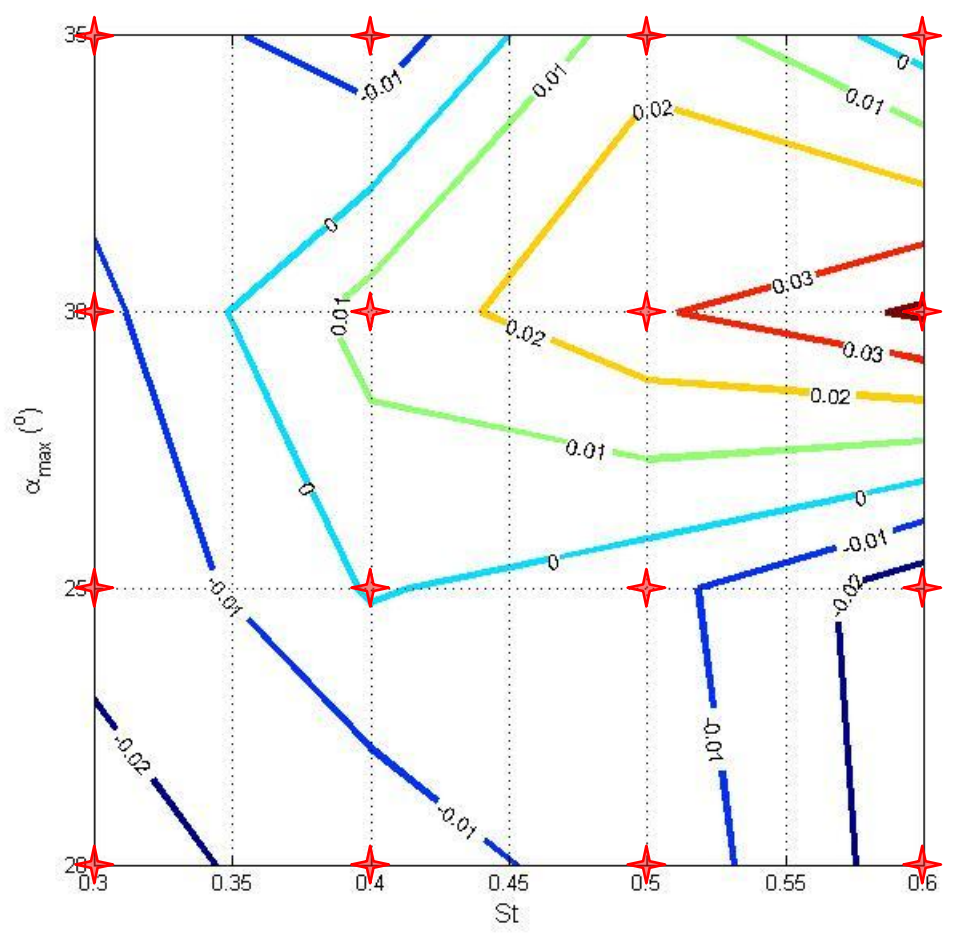

Figure 34. Contour plot for change in mean thrust coefficient

Looking at Figure 32 first, the mean thrust coefficient is positive for the entire plot, which means that there is positive thrust being produced. The same is evident in Figure 33, the case for near the bottom. Again, what is most beneficial is to see the change in mean thrust coefficient from near bottom to freestream conditions, Figure 34. What is evident from the plot is that the largest thrust benefit to being in ground effect is found for a St $=0.6$ and $\alpha_{\max }=30^{\circ}$. Surrounding that data point is the only region that is positive, so there is only going to be a thrust benefit at high St and high $\alpha_{\max }$, which equates to a rapid flapping frequency and mid to low range pitch amplitude. Notice that there are large regions of negative change in mean thrust coefficient, which means that less thrust is produced near the bottom as compared to flapping in the freestream. 
These areas are for all conditions where $\mathrm{St}=0.3$, then for all conditions where $\alpha_{\max }=20^{\circ}$ and $25^{\circ}$, then again for a St $=0.4$ and $\alpha_{\max }=35^{\circ}$. This actually means that there is more drag force near bottom on the foil, than there was in the freestream. Just as observed in (Blevins \& Lauder, 2013), they found that undulating fins generally incur costs from moving close to a solid boundary.

3.3. Results for different series of Strouhal number

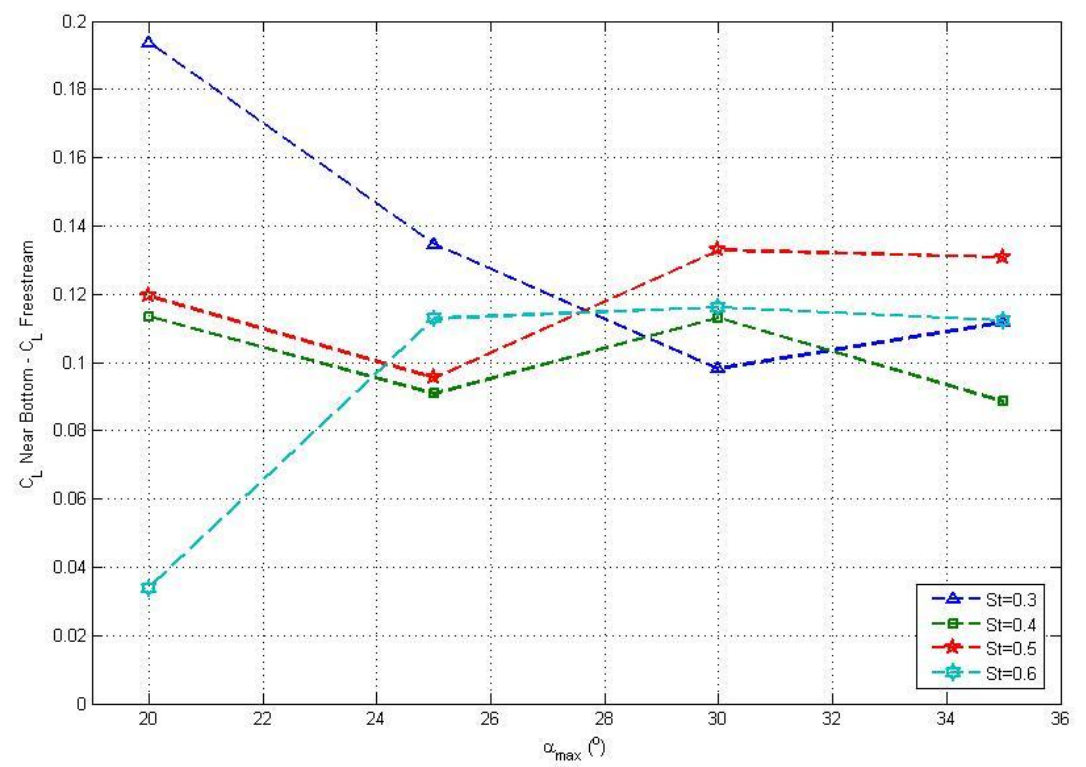

Figure 35. Change in mean lift coefficient as a function of maximum angle of attack

The change in mean lift coefficient for every value of St and $\alpha_{\max }$ in the experimental range is a positive number; mean lift coefficient near bottom is greater than mean lift coefficient in the freestream. For an $\alpha_{\max }$ of $25^{\circ}$ to $35^{\circ}$, the change in mean lift coefficient is in a fairly narrow band. It is only for an $\alpha_{\max }=20^{\circ}$, so largest pitch amplitude and most feathered foil, that the results diverge the most. The results for $\mathrm{St}=0.3$ and $\mathrm{St}=0.6$ have opposite trends, concave 
up, as opposed to concave down. The middle two St have fairly stable trends, such that there does not appear to be much difference to changing the $\alpha_{\max }$ over the range studied. The data in Figure 35 shows what was observed earlier in Figure 31, that if the largest change in mean lift coefficient is sought, it is to be found for $\mathrm{St}=0.3$ and $\alpha_{\max }$ of $20^{\circ}$. Similarly, the smallest change is for a St=0.6 and $\alpha_{\max }$ of $20^{\circ}$.

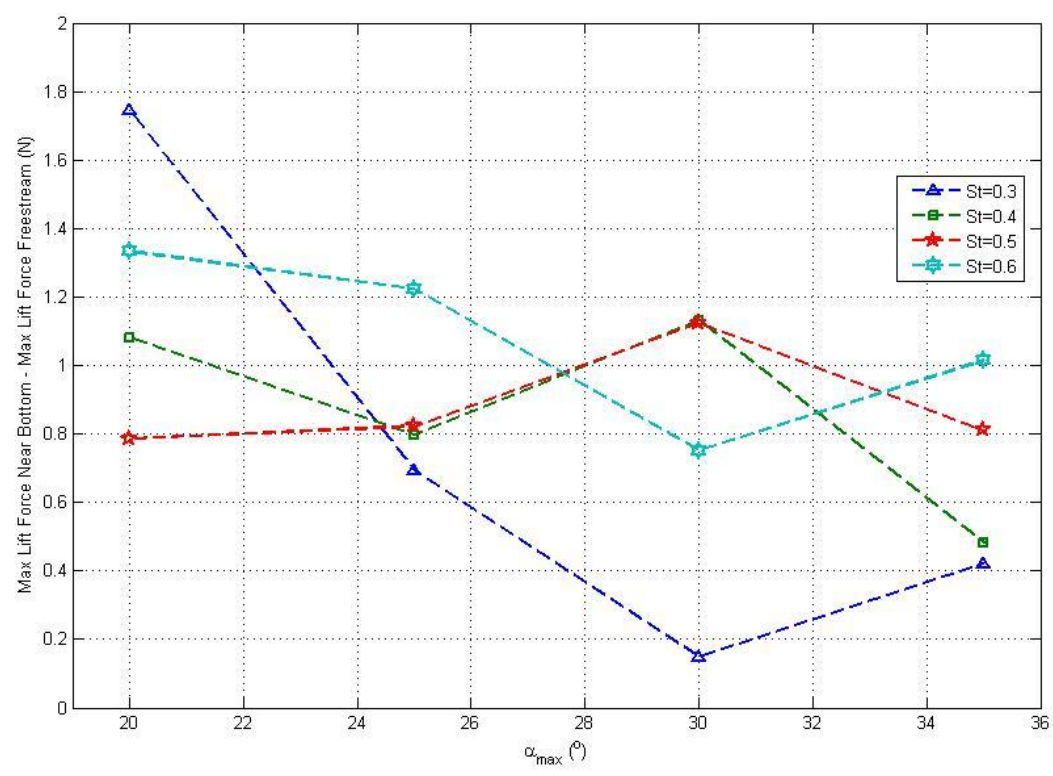

Figure 36. Change in maximum lift force as a function of maximum angle of attack

The figure directly above gives another indication that for all experiments the highest single lift force in ground effect was larger than the highest single lift force in the freestream, proving the lift benefit of ground effect. For St=0.3 and $\alpha_{\max }=20^{\circ}$, the combination of both the largest change in mean lift coefficient, and the largest change in maximum lift force is observed. The percent difference between the maximum lift force near bottom and in the freestream at that point 
is $45 \%$. This seems like a huge advantage, however, from a practical point of view the value of this observation is limited since the foil is producing net drag at this kinematic condition.

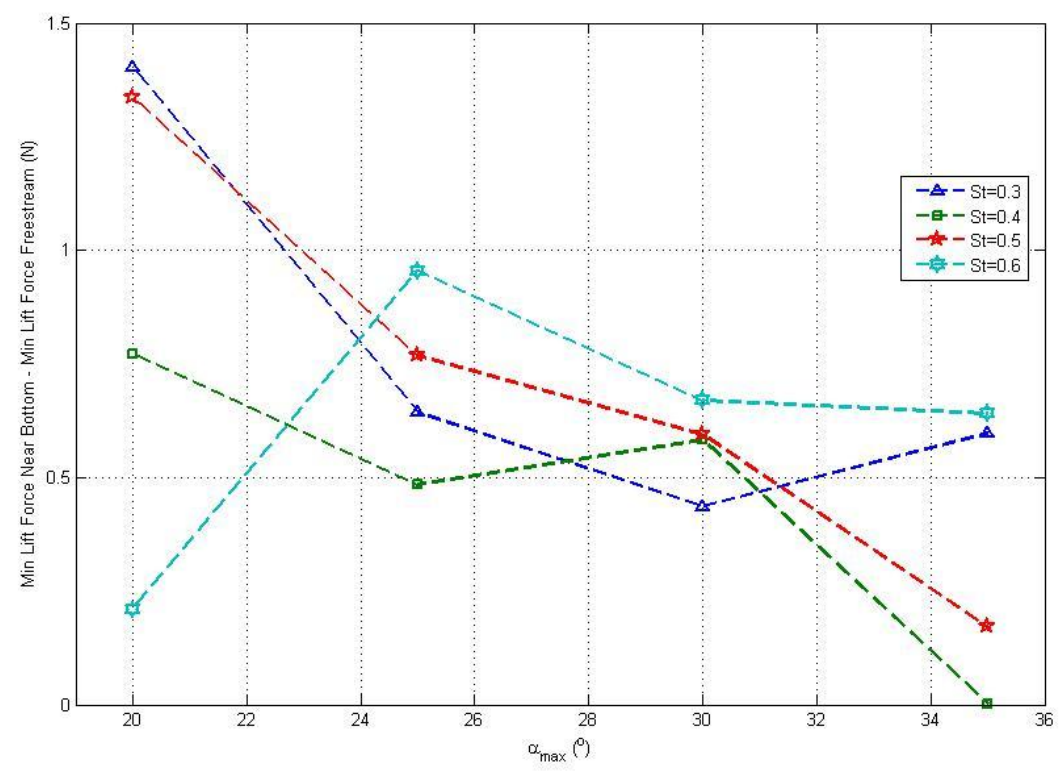

Figure 37. Change in minimum lift force as a function of maximum angle of attack

The magnitude of the change is smaller when considering the minimum observed lift force, but again, all changes observed are positive. In the case of minimum lift force, the positive change means that the minimum lift force due flapping near the bottom is less negative than that due to the freestream, so indicates more beneficial boost to lift. 


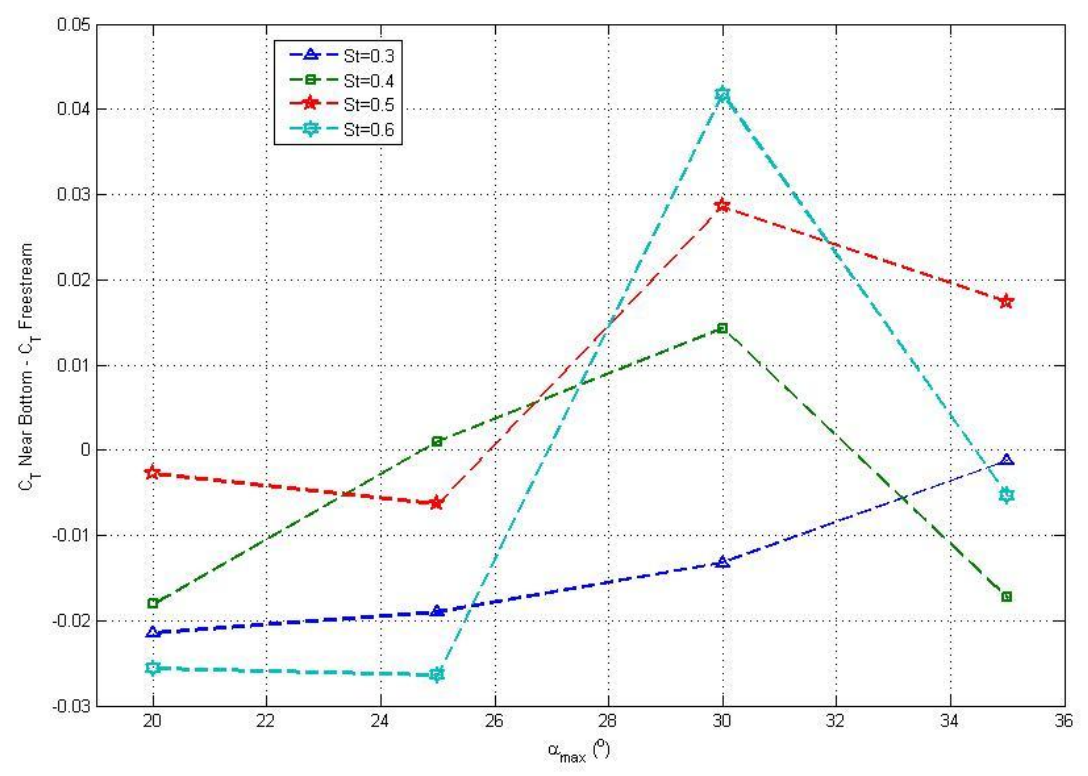

Figure 38. Change in mean thrust coefficient as a function of maximum angle of attack

The figure above clearly illustrates which combinations of St and $\alpha_{\max }$ will generate a thrust benefit due to flapping in ground effect, namely the region where the change in thrust coefficient is positive. The same is shown in the contour plot, Figure 34. If the desired operational conditions of a UUV is to generate more thrust due to flapping near the ground, as compared to flapping in the freestream, that is the area to choose. For $\mathrm{St}=0.3$, more thrust is never produced, all that is obtained is a larger amount of drag for this low frequency flapping. For $S t=0.4$, the mid-range $\alpha_{\max }$ values give a thrust benefit. For the higher St, it is only at higher $\alpha_{\max }$ values that a thrust benefit is seen, but not for the highest $\mathrm{St}=0.6$. 


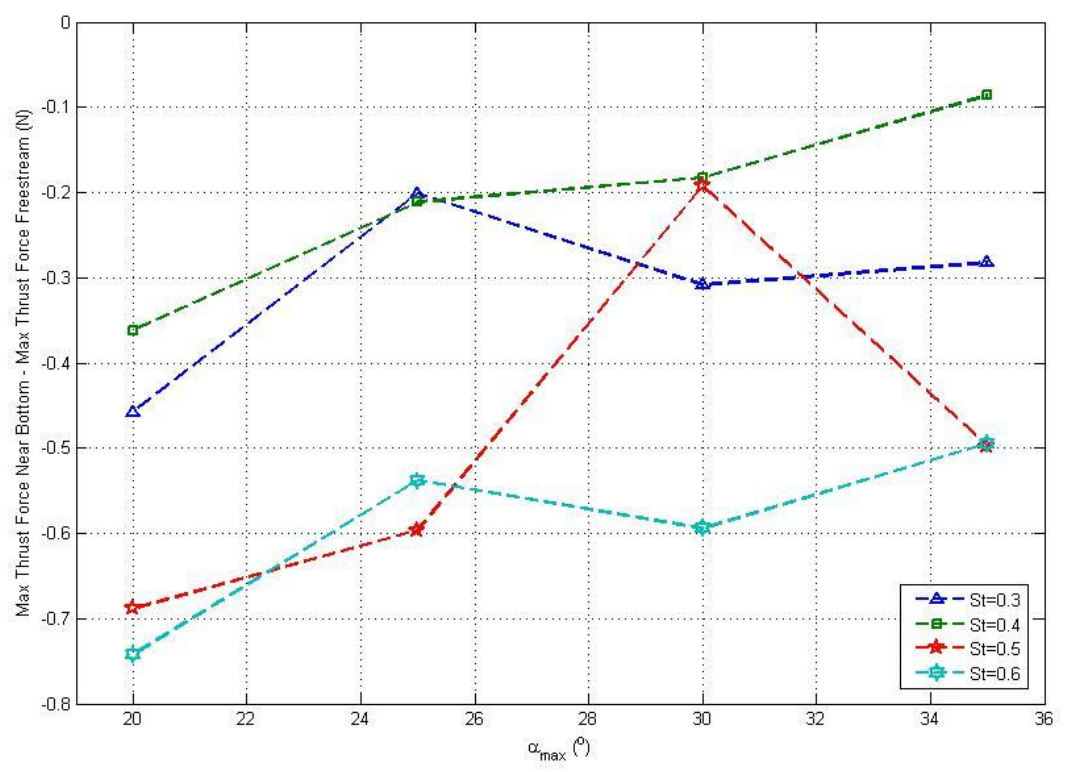

Figure 39. Change in maximum thrust force as a function of maximum angle of attack

The figure above is perhaps a little misleading at first glance. It shows in all cases that the maximum thrust force for flapping near the bottom is less than the value for flapping in the freestream. This situation can still result in an overall positive mean thrust coefficient, as it is calculated by taking the mean thrust force value over the entire flapping cycle, from Equation 7. This will be explored later by observing the phase averaged thrust force curves. 


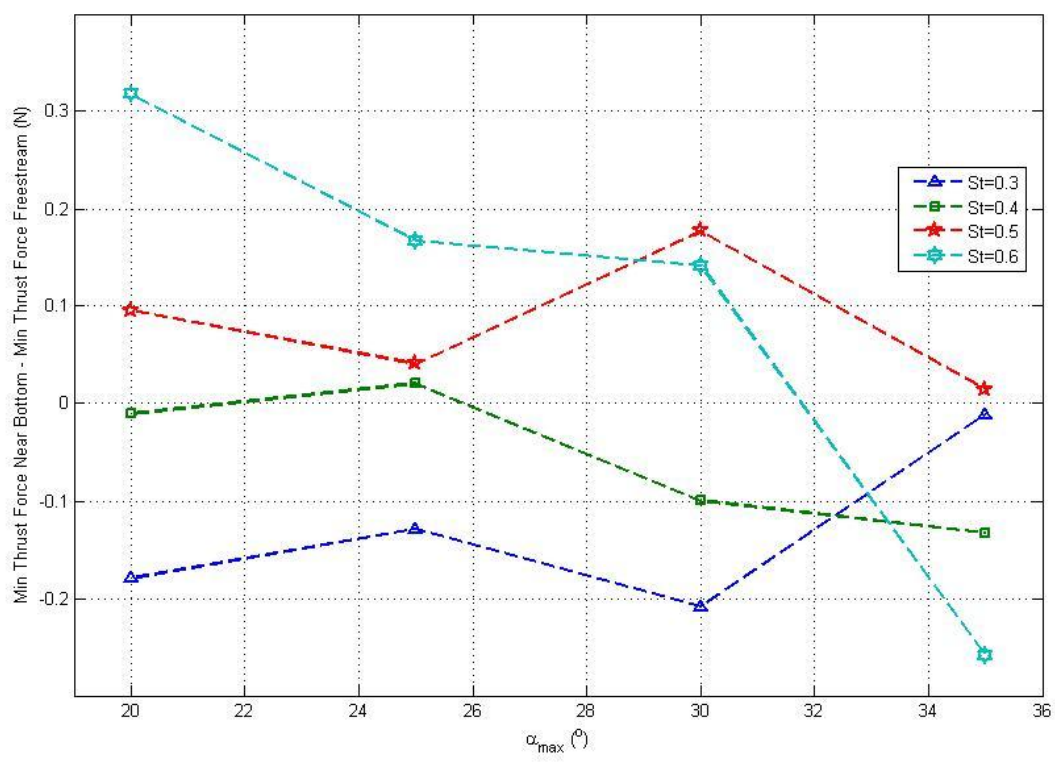

Figure 40. Change in minimum thrust force as a function of maximum angle of attack

The figure above is also slightly misleading, in the same way as Figure 39 was. The interpretation will be revisited after showing phase averaged thrust force curves.

3.4. Results for a single maximum angle of attack

As stated earlier, the procedure to find zero mean lift coefficient by running through a sequence of pitch bias values was conducted at $\mathrm{St}=0.5$ and $\alpha_{\max }=30^{\circ}$. In the data set, there were also three other points at that same $\alpha_{\max }$. These four data points will be used now for a comparison, as St is varied. The results can be followed by referring to the contour plots, and tracing a horizontal line through $\alpha_{\max }=30^{\circ}$. 


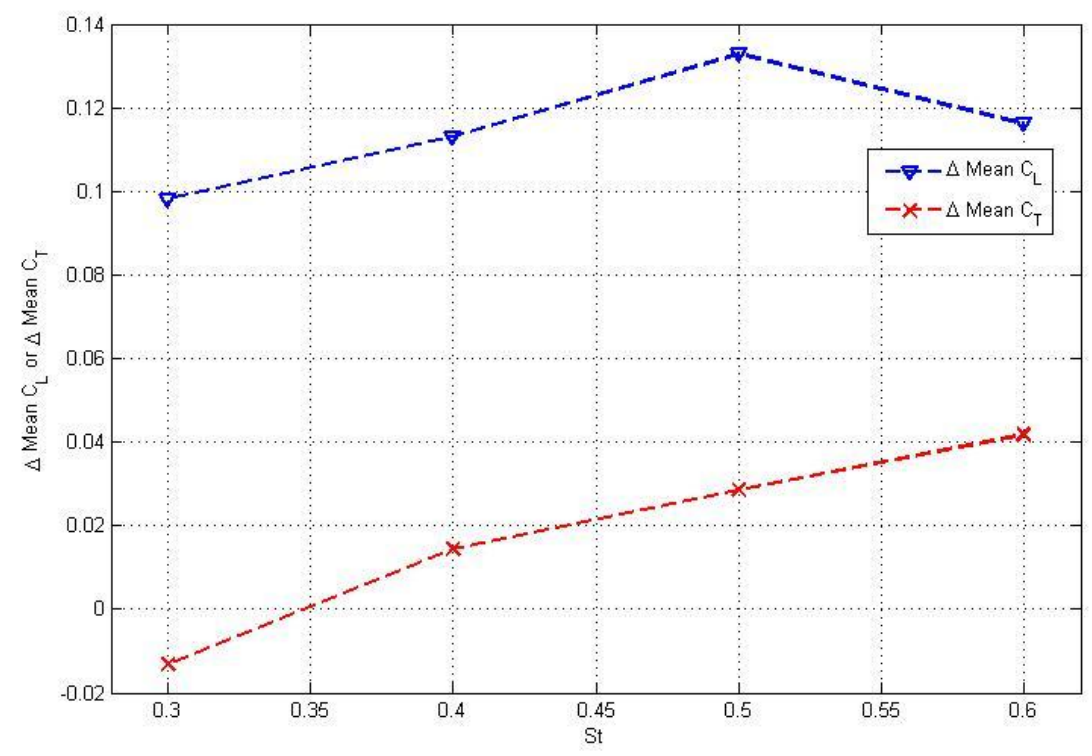

Figure 41. Change in mean lift coefficient or mean thrust coefficient as a function of Strouhal number for maximum angle of attack of $30^{\circ}$

The figure above shows that in all four cases, the change in mean lift coefficient is positive and has a maximum for $\mathrm{St}=0.5$. This means that ground effect is producing a lift benefit. The mean thrust coefficient is positive for the three higher St, and slightly negative for $\mathrm{St}=0.3$. These results agree with the previous numerical study for insect wing flapping in air (Wu, Shu, Zhao, \& Yan, 2014). For all cases the mean lift coefficient increased in ground effect, and the thrust coefficient shows the same pattern, namely that thrust coefficient decreased for low St, and increased for high St. 


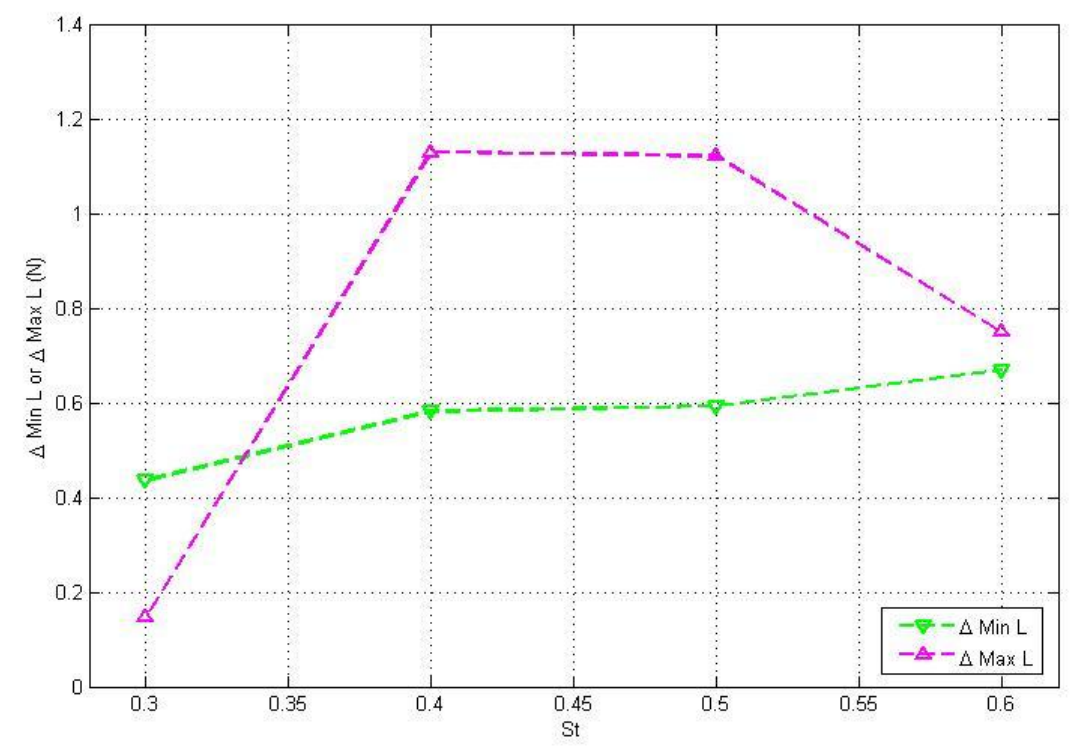

Figure 42. Change in minimum lift force or maximum lift force as a function of Strouhal number for maximum angle of attack of $30^{\circ}$

The figure above shows the lift forces for the four data points. Notice that all points are positive, showing the improvement in lift. The largest changes in max lift force are for $\mathrm{St}=0.4$ and 0.5 .

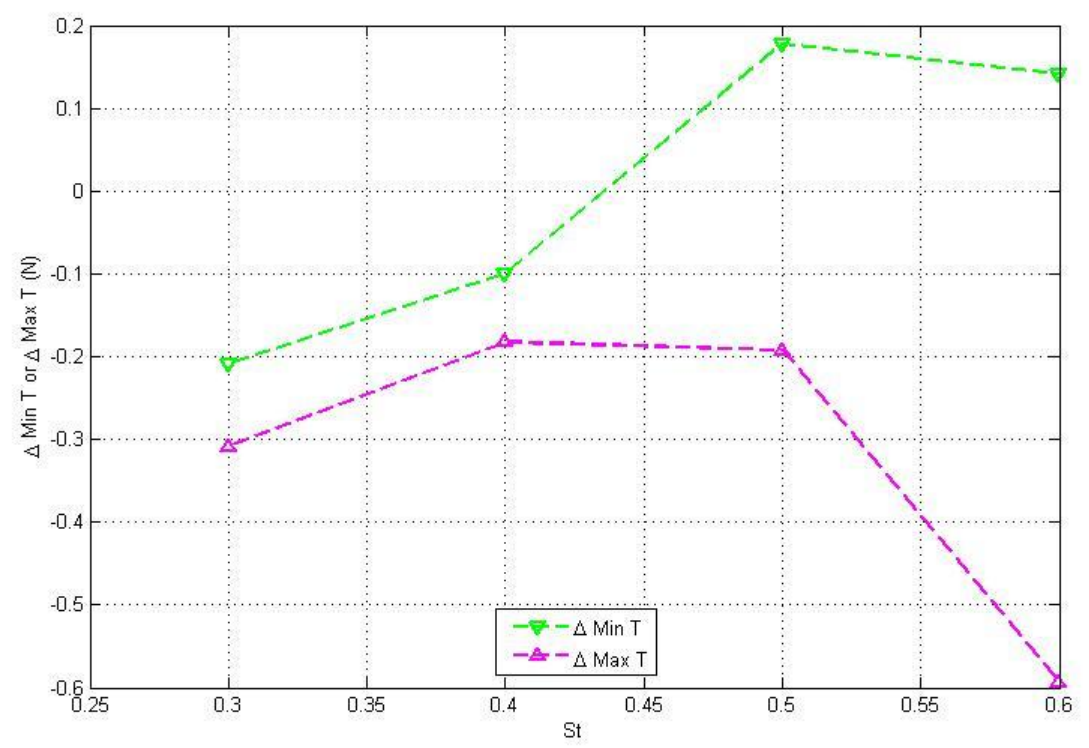

Figure 43. Change in minimum thrust force or maximum thrust force as a function of Strouhal number for maximum angle of attack of $30^{\circ}$ 
The figure above shows the changes in thrust forces, that again may be misleading, but these are simply the maximum and minimum values. The next section will clear up and elaborate.

\subsection{Phase averaged lift and thrust force plots}

The following plots have been produced by taking many flapping cycles and phase averaging them into one upstoke and downstroke, for the freestream and near bottom regimes. Four examples are given for the four data points where $\alpha_{\max }=30^{\circ}$. Dashed lines are for freestream, solid are for near bottom.

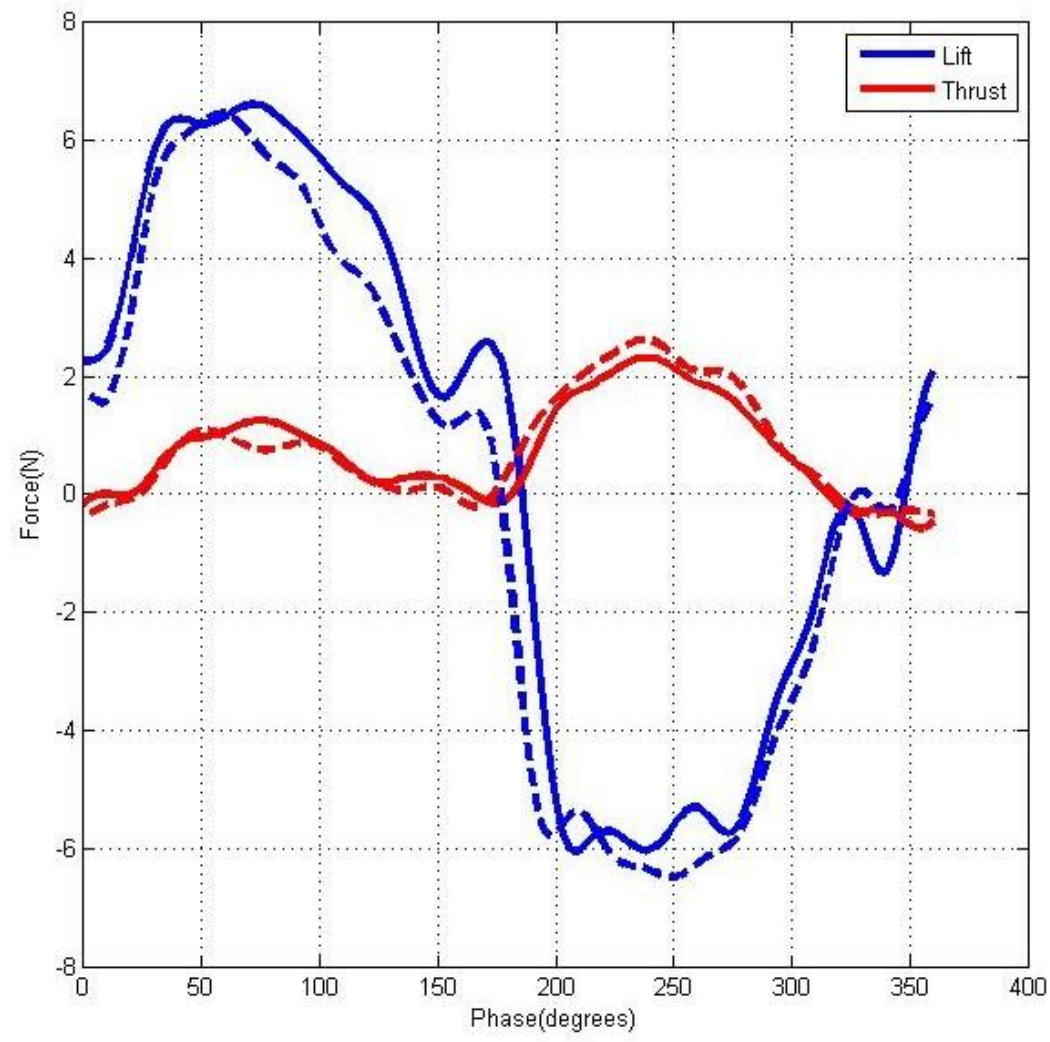

Figure 44. Phase averaged lift and thrust force for $\mathrm{St}=0.3$ and $\alpha \mathrm{max}=30^{\circ}$ (dashed=freestream, solid=near bottom) 
The figure above shows that the lift force is consistently greater in the case of near bottom than it is for the freestream. Notice that the opposite is true for the thrust force, the freestream has a larger positive maximum value. For this case, as seen in Figure 41, the change in mean thrust coefficient is slightly negative, so there is more thrust produced during flapping in the freestream, as compared to near bottom.

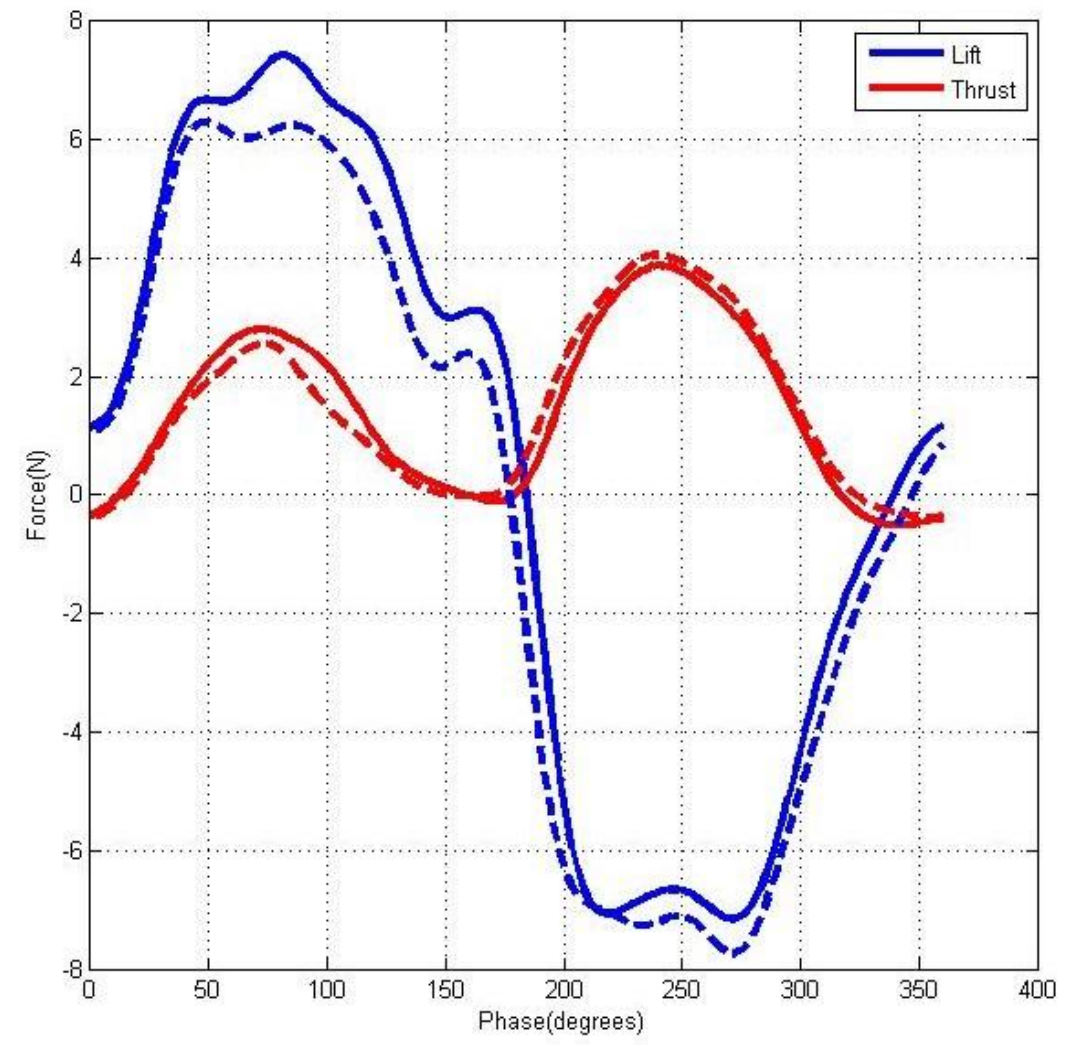

Figure 45. Phase averaged lift and thrust force for $S t=0.4$ and $\alpha \max =30^{\circ}$ (dashed=freestream, solid=near bottom)

Next considering the figure above, again the maximum lift force for near bottom is larger than in freestream, but the maximum thrust force is smaller. For this case of $\mathrm{St}=0.4$, the change in mean thrust coefficient is actually positive, and will 
be for the next two St that follow. This perfectly explains the misleading data from earlier (Figure 39, Figure 40, Figure 43), because here the first peak in thrust has a lot more area underneath it for the near bottom case, and the second peak does not have such a large difference in area underneath. The first peak increase more than makes up for the second peak decrease, and the net result is a larger mean thrust coefficient, as calculated from Equation 7.

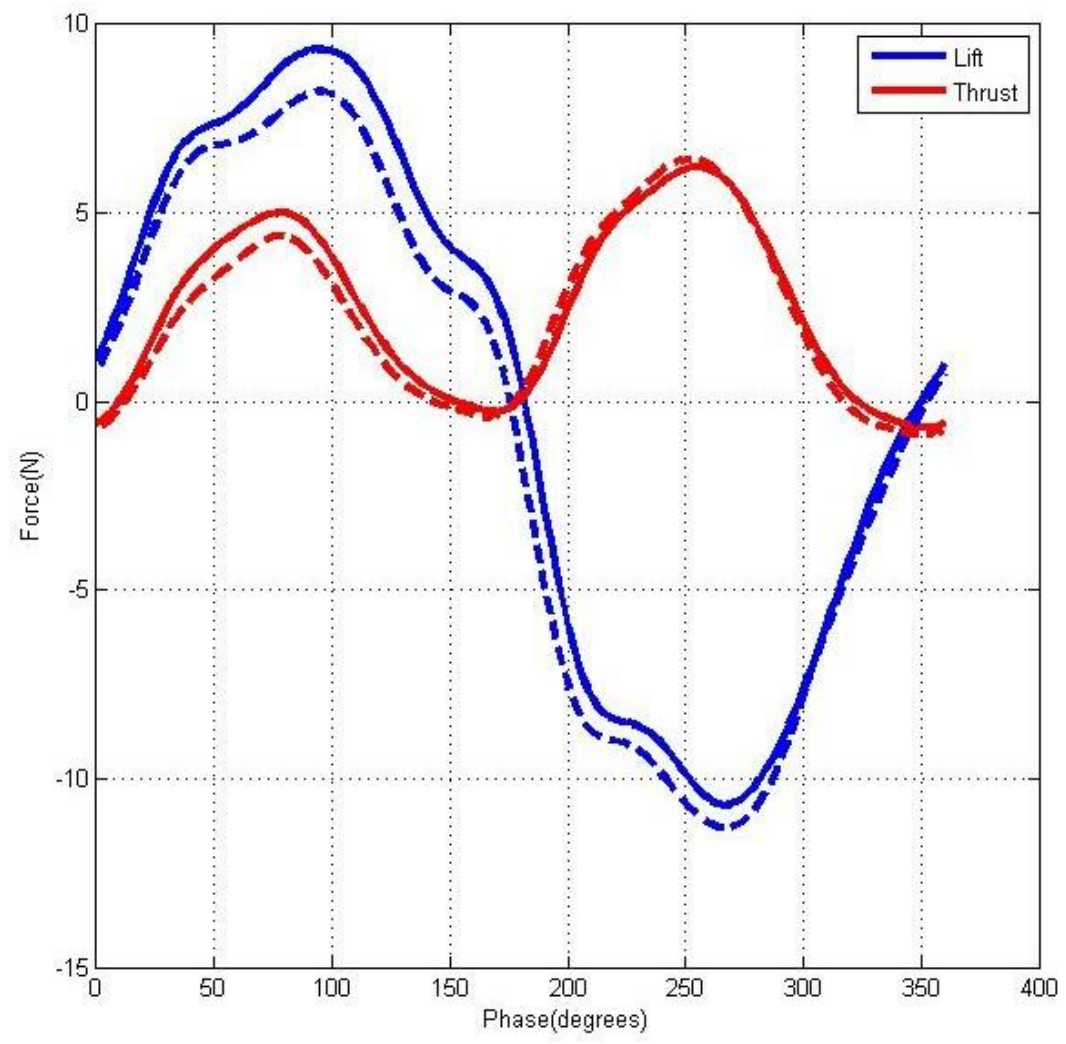

Figure 46. Phase averaged lift and thrust force for $\mathrm{St}=0.5$ and $\alpha \mathrm{max}=30^{\circ}$ ( dashed=freestream, solid=near bottom)

For the figure above, all of the same attributes from the previous two cases are seen. Notice that the peaks in thrust are moving closer to the same height as St is increased. The figure also corresponds to the same kinematic conditions as the lift and thrust plot taken from (Rauworth, 2014), which is Figure 8 in this work. 
Notice the trends in lift and thrust are very similar to Figure 8, though here the magnitude of the traces are a few Newtons of force lower. It is difficult to determine which, if any, of the two examples compared is the correct, true representation of forces generated by the foil, especially since the moment equation procedure led to those forces. The following is simply a side by side comparison showing Figure 8 and Figure 46 again:
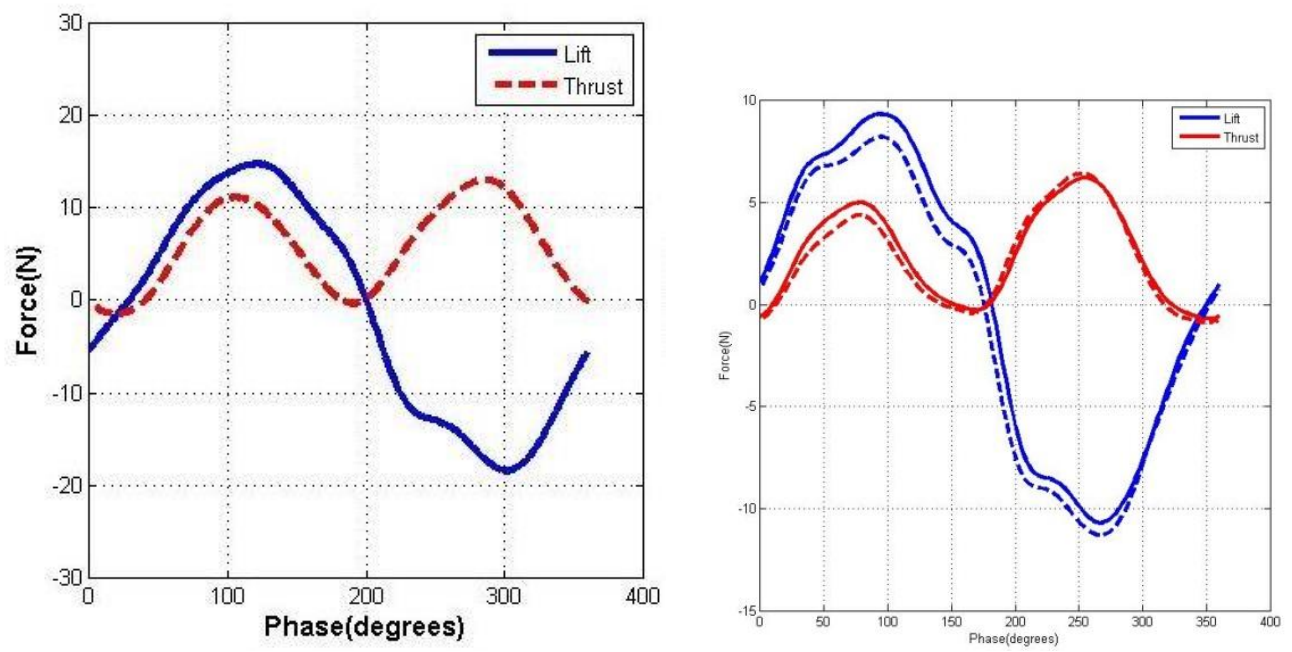

Figure 47. Comparison between lift and thrust plot for (Rauworth, 2014) and this work

What is important is just to note that the trends are the same, and that in the current work, an influence in the data from the foil being near bottom was seen. Phase averaging was conducted slightly differently for both plots, so the expectation is that the lift and thrust traces would not line up for perfect side by side comparison. 


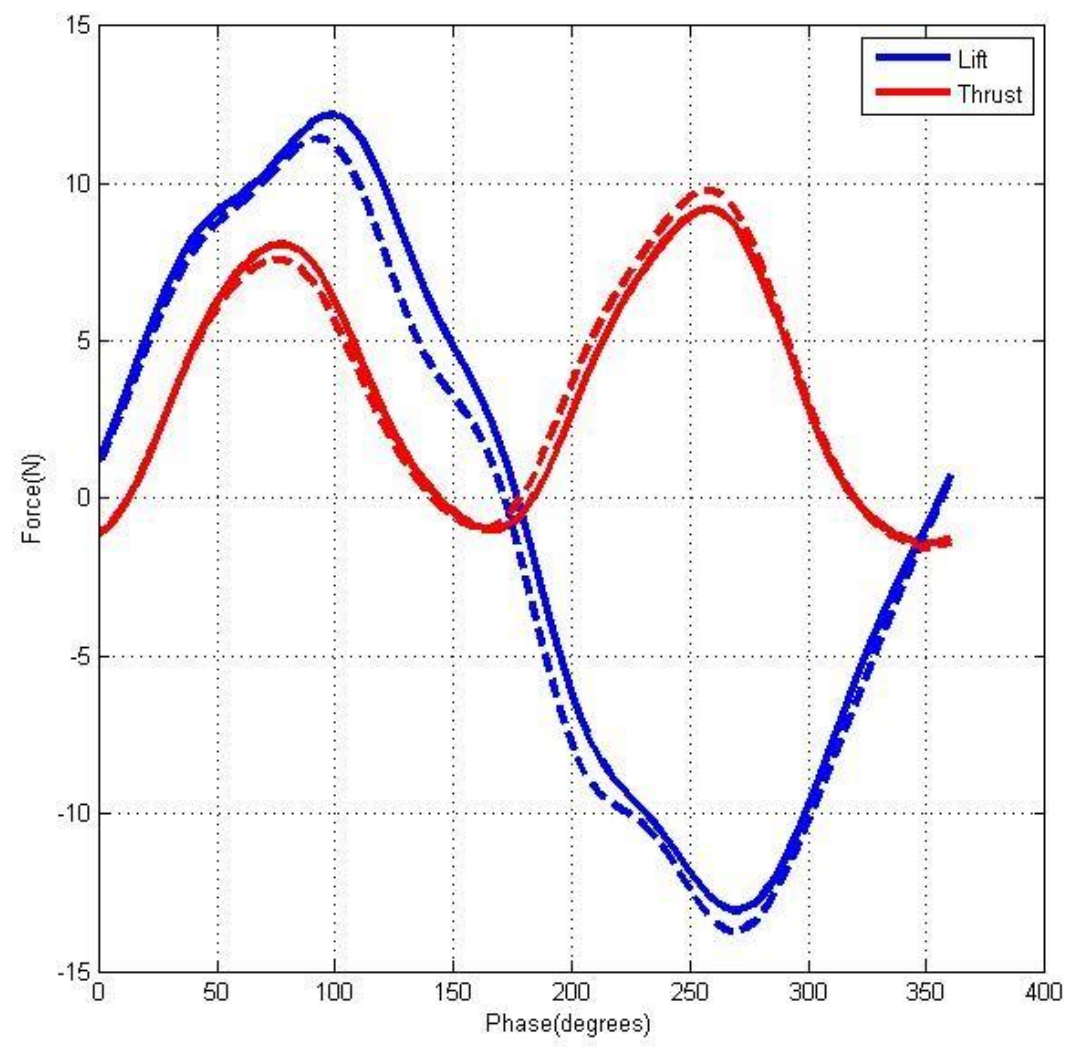

Figure 48. Phase averaged lift and thrust force for $S t=0.6$ and $\alpha \max =30^{\circ}$ (dashed=freestream, solid=near bottom)

For the figure above, notice that the near bottom lift trace is symmetrical. For this particular case, it happens to be that the largest change in mean thrust coefficient is observed in the entire data set. The percent difference between maximum thrust force near bottom and in the freestream is $6 \%$, so if an overall thrust benefit due to ground effect is sought, that change in instantaneous maximum thrust force will need to be sensed. The percent difference in mean thrust coefficient here was $8 \%$. Of course, this may not be as useful as it seems, because the propulsive efficiency difference has not been calculated. Similar to findings in (Blevins \& Lauder, 2013), the power requirement may increase at this kinematic condition when flapping near the bottom. 
4. Design Improvements and Error Analysis

\subsection{Design Improvements}

The experiments indicate that despite improvements in signal to noise ratio over previous apparatus, it will still be necessary to change the design inside the pitch canister to achieve quantifiable, repeatable results.

\subsubsection{One force sensor}

The most ideal method of force sensing would be to only have one sensor, instead of the two that are currently installed. One larger bearing and sensor could be installed to support the pitch shaft, and there would no longer be confusion about which direction forces were acting in, and having to resolve both force sensor forces into one. Also, in the x- and y-directions the sensors had the same range, but a different range of sensing existed for the z-direction. Forces in the z-direction were very important, as they lead to the lift calculations for the foil.

\subsubsection{Install an accurate torque sensor}

A torque sensor should be installed on the pitch shaft. With the present setup, there is a measure of torque output based on motor current for the roll and pitch motors, but the data rate and resolution is too low to be useful. A new torque sensor will give a measure of the power required to drive the foil. From that data the propulsive efficiency can be determined to see if flapping near the bottom is more efficient. Those kinematic conditions for which a propulsive efficiency boost due to flapping near the bottom can be investigated and employed on future UUVs. 


\subsection{Sources of error}

\subsubsection{Method for centering foil in roll}

The foil was pulled up to the support bracket limit, and then commanded to rotate 5200 counts down in roll, in order to start it at the horizontal. This method, used in (Rauworth, 2014), likely introduced some error.

\subsubsection{Pitch bias sweep to find zero mean lift coefficient}

In future tests, a zero mean pitch bias should be found for flapping in the freestream, rather than near bottom. That value for pitch bias should also be used, to two decimal places rather than closest integer, for all experiments. By doing that, the "normal" condition would be flapping in the freestream, and then any ground effect by flapping near the bottom would be seen clearly in the data.

\subsubsection{Carriage drive mechanism and dual canister motors}

Another source of error may be found in the carriage drive mechanism, which is old and fairly unreliable. The LDM should be connected to the drive mechanism, in order to command the carriage to move at precise speeds, rather than just using a rheostat.

\subsubsection{Motor control}

Foil position has been plotted based on the input command, so the desired position was used. As mentioned earlier, the roll and pitch motors of the dual canister are old, and their actual output position data was not used. A potential source of error is therefore introduced if the desired and actual motor positions are different. 


\section{Future Work}

\subsection{Stationary carriage}

The testing platform and processing software was only setup to run at some non-zero forward speed. It would be valuable to determine the flapping conditions required for station keeping, such that a high lift is required, and basically zero thrust. Station keeping for a UUV may be employed during swimmer defense, explosive ordinance disposal, video surveys of one specific point of interest, and many other missions.

\subsection{Under a free water surface}

Just as a lift and thrust benefit was observed close to a hard, bottom surface, an investigation could be conducted into the effects of flapping near a free surface. Although not hard, there is a drastic density change between water and air, so it would be a worthy pursuit. However, the wave making that is the result of flapping near the free surface might make potential gains obsolete, by wasting energy. The damping force that would be produced would dissipate energy.

\subsection{Under wave conditions}

Real world deployment of UUVs is of ultimate importance for all this investigation work, and in the real world there are waves to deal with in any significant body of water. The tow tank that testing was conducted in has a wave generator, and the foil performance could be tested under diverse wave 
conditions to see if there are particular wave conditions which provide benefits to various aspects of a UUV mission.

\subsection{Near walls}

Similar to flapping near a horizontal bottom ground, flapping near vertical walls or even under hard surfaces should be explored. The flapping could be oriented to have a flap centerline to match the profile of the hard surface, or to flap with the tip of the foil perpendicular to the hard surface in question. The two different configurations would allow either most of the span to interact with the hard surface, or just the tip so that end effects could be investigated. Flapping perpendicular to a hard surface may provide similar benefits to comparing three dimensional flow to two dimensional flow over a wing. Tip losses are minimized by vertical winglets at the wing tips in aerospace applications, a similar benefit remains to be investigated underwater.

\subsection{Performance and operational considerations}

In future work many more parameters to find the kinematic conditions in which a benefit from ground effect is observed must be used, such as power consumption and propulsive efficiency. A much more comprehensive experimental matrix can be employed to get more resolution by varying St and $\alpha_{\max }$ in finer increments. Again using nature as an inspiration, observations from flying animals utilizing ground effect can lead to some operating rules for UUVs to maximize the benefit. Some animal behaviors have been identified as: (i) an animal cannot fly very slowly in ground effect; (ii) for power or range economy 
an animal should reduce speed when commuting in ground effect; (iii) an animal should show less tendency to vary flight speed at any given height when in ground effect, particularly when it is flying slowly, at or around the minimum power speed; and (iv) when varying flight speed over a surface an animal should reduce height at higher flight speeds (Rayner, 1991). The same paper discussed even more observations from nature. Pelicans gliding over water fly at a much lower altitude $(\mathrm{H} / \mathrm{s}=0.31)$ than when they are flapping $(\mathrm{H} / \mathrm{s}=0.50)$. Notice that the author used span to non dimensionalize the altitude. Skimmers and myotid bats adopt an asymmetric wingbeat in which the downwards excursion of the wing below the body has a much lower amplitude than the upper half of the wingbeat. In this work an equal upstroke and downstroke roll amplitude was used, but future work can certainly investigate increasing the roll amplitude for the foil during the upper half of the flap, when it is away from the ground.

As far as animals swimming in water near the bottom, some observations have been made by (Blevins \& Lauder, 2013). They noted that live animals change their behavior when swimming close to a solid boundary. Due to the stiffness of the fin model used, the authors were not able to detect subtle influences that ground effect has on fin shape. They noted that kinematic changes observed in fish swimming in ground effect are due to active modulation, not passive effects. Furthermore, these changes consisted of reduced tailbeat frequency and amplitude, which may be a mechanism for transforming potential locomotor penalties into performance benefits. Stingrays have extremely fine control of pectoral fin conformation with the potential for precise kinematic tuning to exploit ground effect. 
These behavior changes noted in animals exploiting ground effect may provide a starting point for more investigation into this topic. Of course these behaviors will have to be programmed into the UUV profile in order to take maximum advantage of ground effect. When the UUV is required to go into ground effect, an appropriate signal can be used to sense when it is there, such as maximum lift force. The UUV will then be able to modify the St, $\alpha_{\max }$, or foil roll amplitude accordingly to mimic the behavior from nature, if it leads to better efficiency. The change in signal strength will have to be able perceived in future UUVs, and will be used in a control feedback loop to keep the vehicle in ground effect, as desired. 
6. Summary and Conclusions

It has been proven that there is a quantifiable benefit in terms of lift and thrust to be seen when flapping a foil close to the ground, under certain kinematic conditions. Much work remains to be done to investigate the benefit and to take advantage of it on vehicles that use flapping foils for propulsion. This investigation provided the best possible starting point, as a three dimensional foil was used on an actuation system that closely resembles a real underwater vehicle. 


\section{Appendices}

\section{Appendix 1. MATLAB code to display results, "ResultsThesis.m”.}

\section{Authored by P. Chierico}

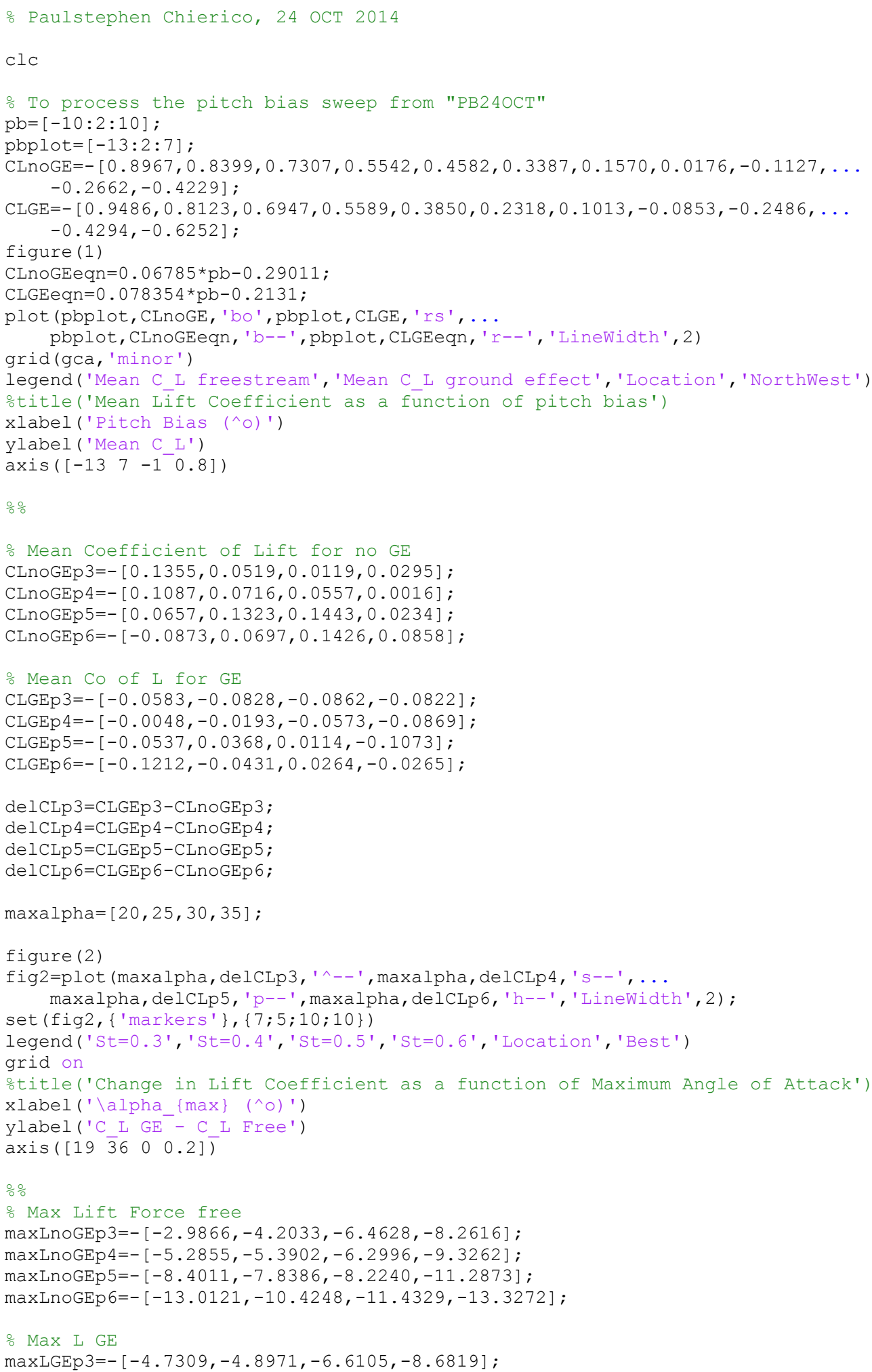




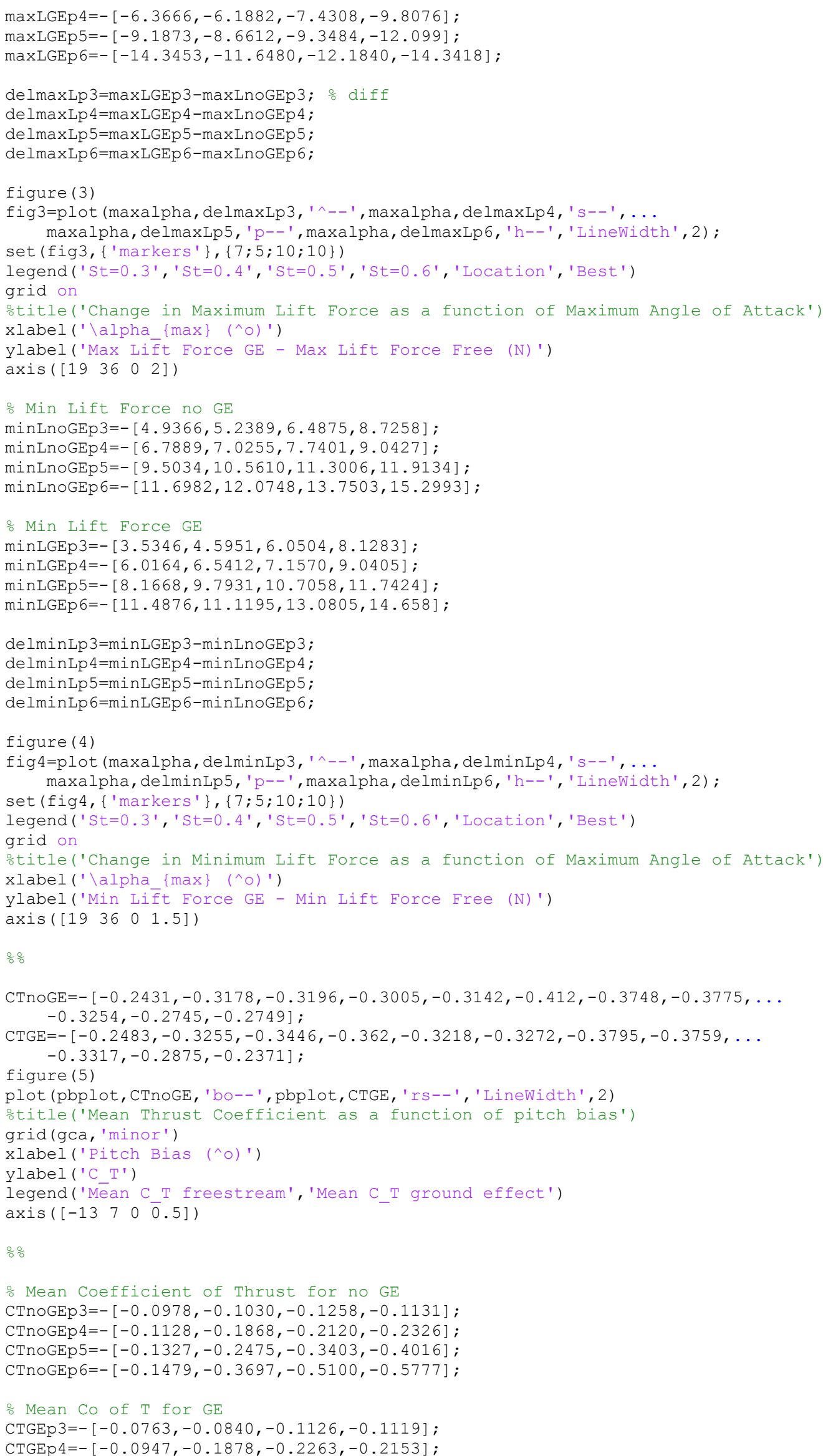


CTGEp $5=-[-0.1299,-0.2412,-0.3689,-0.4190] ;$

CTGEp $6=-[-0.1223,-0.3433,-0.5518,-0.5723]$;

delCTp3=CTGEp3-CTnoGEp3;
delCTp4=CTGEp4-CTnoGEp4;
delCTp5=CTGEp5-CTnoGEp5;
delCTp6=CTGEp6-CTnoGEp6;

figure (6)

fig6=plot (maxalpha, delCTp3, '^-- ' , maxalpha, delCTp4, 's-- ',...

maxalpha, delCTp5, 'p-- ', maxalpha, delCTp6, 'h-- ', 'LineWidth' ,2) ; set (fig6, \{'markers' $\},\{7 ; 5 ; 10 ; 10\}$ )

legend ('st=0.3',' 'st=0.4',' 'st=0.5',' St=0.6', 'Location', 'Best' )

grid on

otitle('Change in Thrust Coefficient as a function of Maximum Angle of Attack')

xlabel ('\alpha $\{\max \}\left({ }^{\wedge} 0\right)$ ')

ylabel ('C T GE - C T Free')

axis $([19 \overline{3} 6-0.03 \overline{0} .05])$

\% Max Thrust Force free

maxTnoGEp3 $=-[-2.2178,-2.3124,-2.6190,-2.7647]$;

$\operatorname{maxTnOGEp} 4=-[-3.1119,-3.4356,-4.0593,-4.3017]$;

maxTnoGEp5 $=-[-5.4255,-5.9517,-6.4193,-6.9927]$;

maxTnoGEp $6=-[-8.9669,-8.7929,-9.7747,-10.3269]$;

\% Max thrust force GE

$\operatorname{maxTGEp} 3=-[-1.7600,-2.1112,-2.3105,-2.4823]$;

$\operatorname{maxTGEp} 4=-[-2.7500,-3.2243,-3.8768,-4.2156]$

maxTGEp5 $=-[-4.7371,-5.3553,-6.2268,-6.4947]$;

maxTGEp $6=-[-8.2244,-8.2547,-9.1805,-9.8320]$;

delmaxTp3=maxTGEp3-maxTnoGEp3;

delmaxTp4=maxTGEp4-maxTnoGEp4;

delmaxTp5=maxTGEp5-maxTnoGEp5;

delmaxTp 6=maxTGEp6-maxTnoGEp6;

figure (7)

fig7=plot (maxalpha, delmaxTp3, '^-- ' , maxalpha, delmaxTp4, 's-- ', ...

maxalpha, delmaxTp5, 'p-- ', maxalpha, delmaxTp6, 'h-- ', 'LineWidth ', 2) ;

set (fig7, \{'markers' $\},\{7 ; 5 ; 10 ; 10\}$ )

legend ('st=0.3',' St=0.4','st=0.5', 'st=0.6', 'Location', 'Best' )

grid on

otitle('Change in Maximum Thrust Force as a function of Maximum Angle of Attack')

xlabel (' $\backslash$ alpha_ $\max \}\left({ }^{\wedge} \mathrm{O}\right)$ ')

ylabel('Max Thrust Force GE - Max Thrust Force Free (N) ')

axis ( $\left.\left[\begin{array}{llll}19 & 36 & -0.8 & 0\end{array}\right]\right)$

\% Min Thrust force free

minTnoGEp3 $=-[0.4861,0.4645,0.3701,0.4076]$;

minTnoGEp4 $=-[1.0492,0.6237,0.4057,0.4163]$;

minTnoGEp $5=-[1.6996,1.1352,0.8612,0.6536]$;

minTnOGEp $6=-[4.1461,1.9887,1.5649,0.9520]$;

\% Min Thrust GE

minTGEp3 $=-[0.6656,0.5932,0.5787,0.4189] ;$

minTGEp $4=-[1.0600,0.6034,0.5055,0.5491]$;

minTGEp $5=-[1.6039,1.0946,0.6837,0.6389]$

minTGEp $6=-[3.8285,1.8215,1.4235,1.2111]$;

delminTp3=minTGEp3-minTnoGEp3;

delminTp4=minTGEp4-minTnoGEp4 ;

delminTp5=minTGEp5-minTnoGEp5;

delminTp 6=minTGEp 6-minTnoGEp6;

figure (8)

fig $8=$ plot (maxalpha, delminTp3, '^-- ' , maxalpha, delminTp4, 's--' , ...

maxalpha, delminTp5, 'p--' , maxalpha, delminTp6, 'h--' ', LineWidth ',2) ;

set (fig8, \{'markers' $\},\{7 ; 5 ; 10 ; 10\}$ )

otitle('Change in Minimum Thrust Force as a function of Maximum Angle of Attack')

legend ('St=0.3',' St=0.4',' St=0.5', 'St=0.6', 'Location', 'Best' )

grid on

xlabel ('\alpha_ $\max \} \quad\left({ }^{\circ} \mathrm{O}\right)$ ')

ylabel('Min Thrust Force GE - Min Thrust Force Free (N) ')

$\operatorname{axis}\left(\left[\begin{array}{llll}19 & 36 & -0.3 & 0.4\end{array}\right)\right.$ 


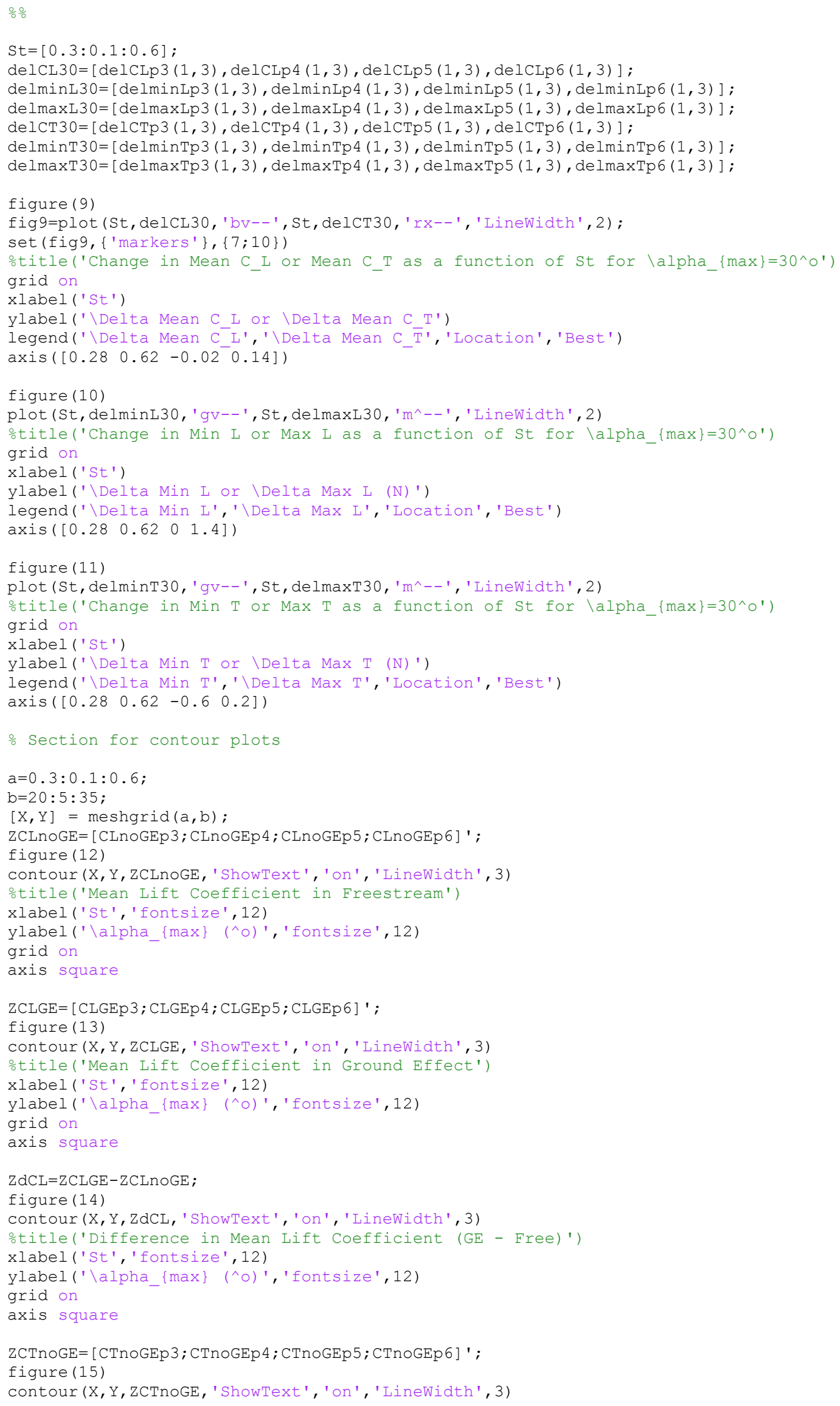


otitle('Mean Thrust Coefficient in Freestream')

xlabel('st','fontsize',12)

ylabel ('\alpha_\{max\} (^o)','fontsize', 12)

grid on

axis square

ZCTGE $=[$ CTGEp3; CTGEp4;CTGEp5;CTGEp6] ' .

figure (16)

contour (X, Y, ZCTGE, 'ShowText' ', on ', 'Linewidth ', 3)

otitle('Mean Thrust Coefficient in Ground Effect')

xlabel ('st', 'fontsize', 12)

ylabel ('\alpha $\{\max \}\left({ }^{\prime} 0\right)$ ','fontsize',12)

grid on

axis square

ZdCT=ZCTGE-ZCTnOGE ;

figure (17)

contour (X, Y, ZdCT, 'ShowText' ', 'on ', 'LineWidth ', 3)

otitle('Difference in Mean Thrust Coefficient (GE - Free)')

xlabel ('st', 'fontsize', 12)

ylabel ('\alpha $\{\max \}$ (^o)','fontsize',12)

grid on

axis square 


\section{Appendix 2. MATLAB code for phase averaging, "PhaseAvg0.m".}

\section{Authored by Professor S. Licht}

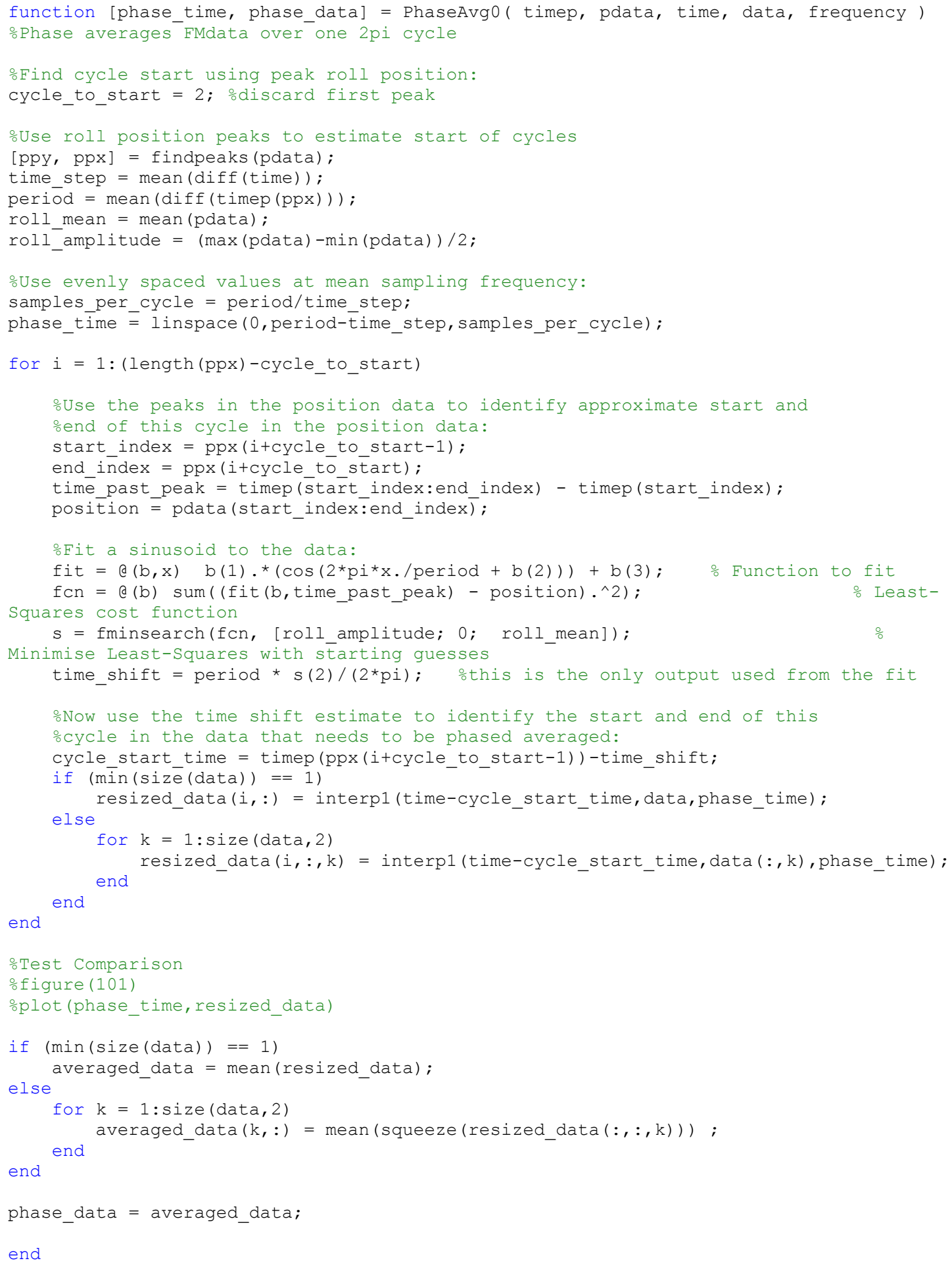


Appendix 3. MATLAB code for finding force and moment data, "Parse2FM0.m".

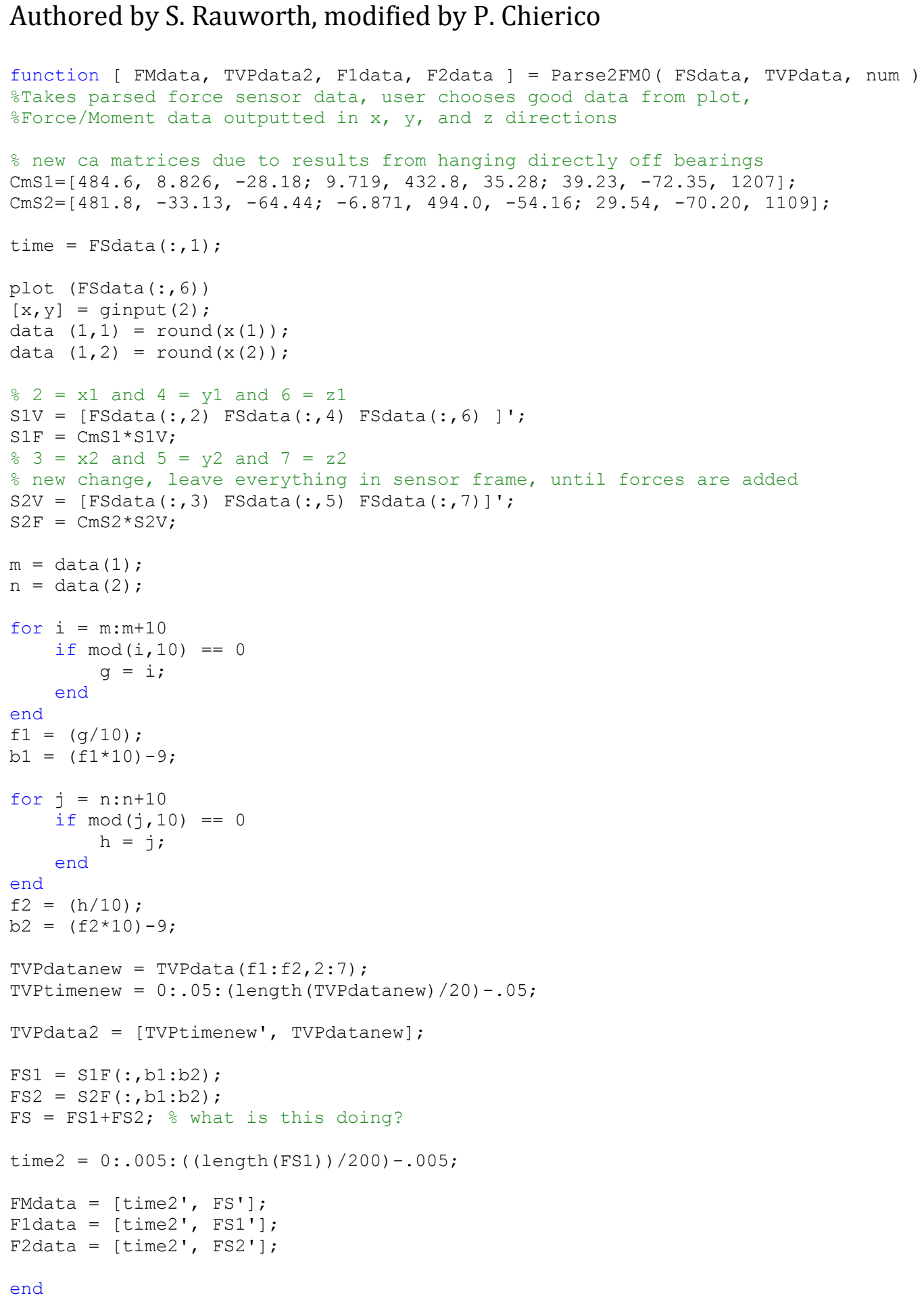


Appendix 4. MATLAB code for filtering, "BFilt0.m”.

Authored by S. Rauworth, modified by P. Chierico

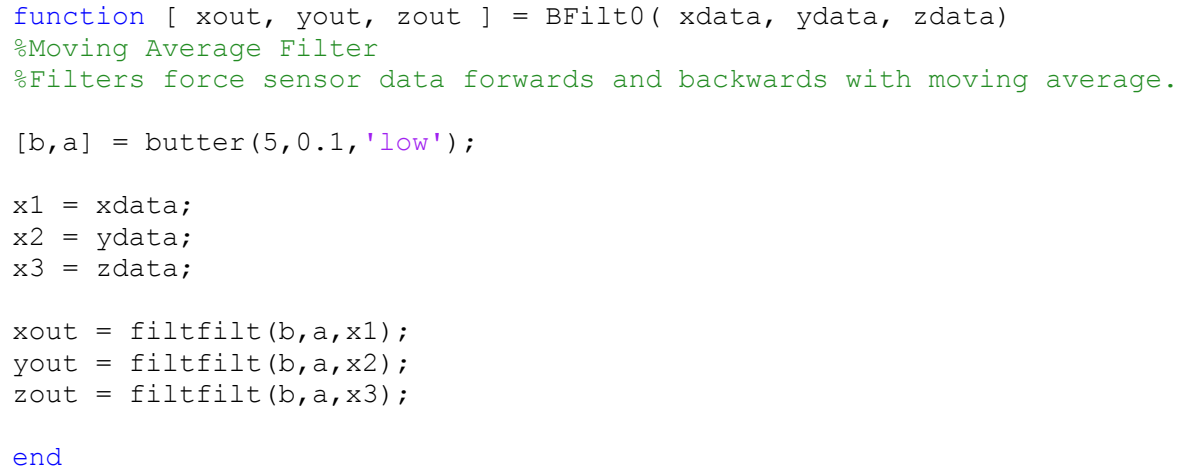




\section{Appendix 5. MATLAB code for analyzing the data, "Analysis0.m”.}

\section{Authored by S. Rauworth, modified by P. Chierico}

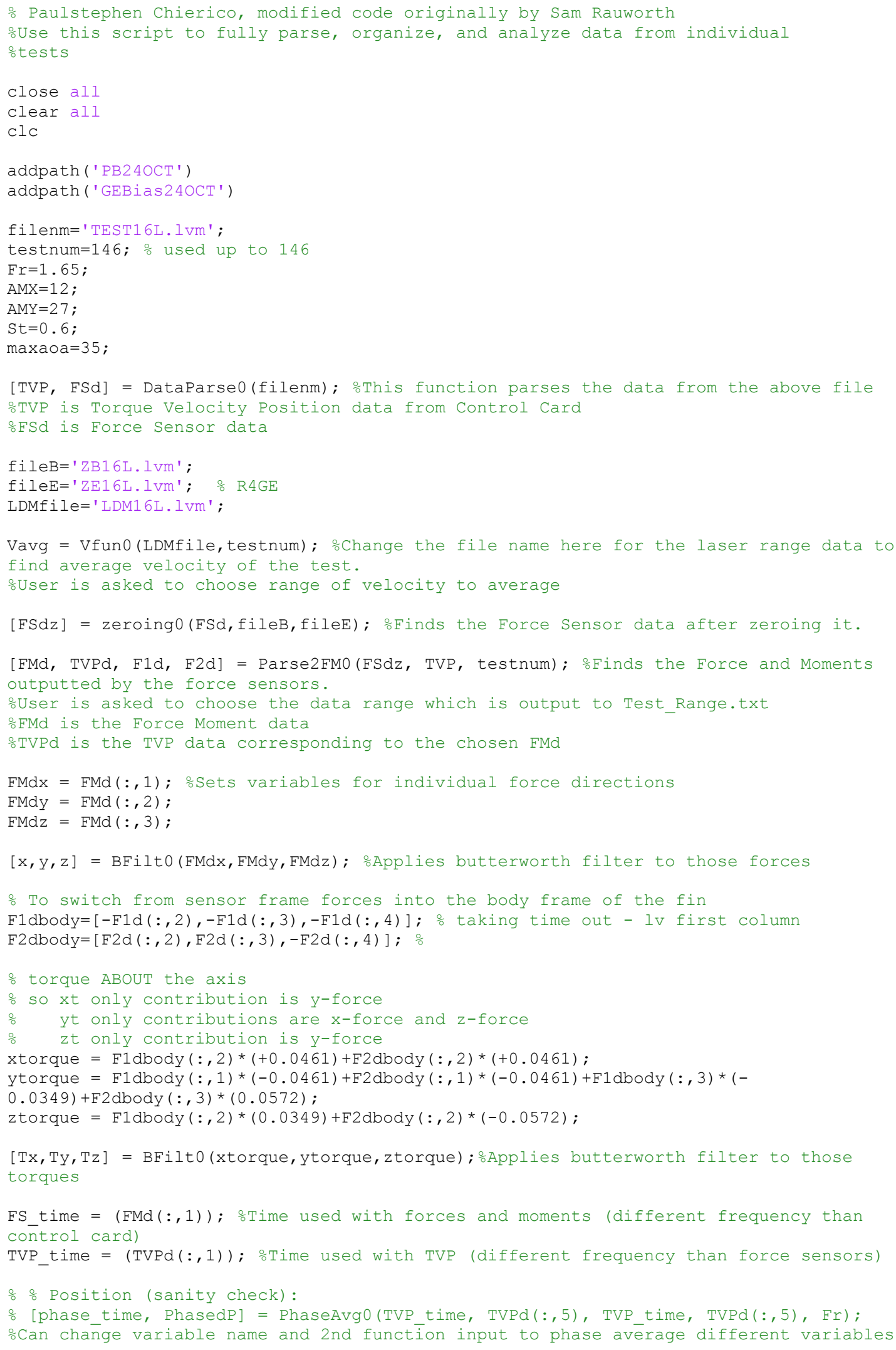


: plot (phase_time, PhasedP)

\% title('Position vs. Phase Sanity Check')

[phase time, PhasedTy] = PhaseAvg0 (TVP time, TVPd $(:, 5)$, FS time, Ty, Fr);

[phase_time, PhasedTz] = PhaseAvg0(TVP_time, TVPd $(:, 5)$, FS_time, Tz, Fr);

Lift $=$ PhasedTy/0.435; \%Converts phase averaged torques into forces by dividing out moment arm or distance from axis of rotation to center of pressure

Thrust $=$ PhasedTz/0.435; $\%$ Min CoP is $.155 \mathrm{~m}$, max is $.555 \mathrm{~m}$, 70 percent is $.435 \mathrm{~m}$

for $i=1$ : length(Thrust) \% The below removes the NaN values from the thrust and lift matrices

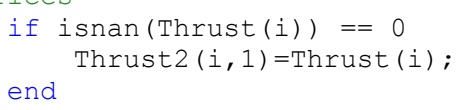

end

for $i=1:$ length (Lift)

if isnan (Lift(i)) $==0$

Lift $2(i, 1)=$ Lift $(i)$;

end

end

\% To find the mean thrust coefficient

Ct $=(2 *$ mean $($ Thrust 2$)) /\left(1000 *\left(\operatorname{Vavg}^{\wedge} 2\right) * .1 * .4\right) ;$

$\mathrm{Cl}=(2 * \operatorname{mean}(\operatorname{Lift} 2)) /\left(1000 *\left(\operatorname{Vavg}^{\wedge} 2\right) * .1 * .4\right)$

MinLift=min (Lift);

MaxLift=max (Lift);

MinThrust=min (Thrust) ;

MaxThrust=max (Thrust);

\% Change file name and structure name for different testing sets

load ('PstTests.mat')

$\%$

Struct (testnum). name = filenm;

Struct (testnum). St $=$ St;

Struct (testnum). maxaoa = maxaoa;

Struct (testnum). Freq = Fr;

Struct (testnum). $\mathrm{AMX}=\mathrm{AMX}$;

Struct (testnum). AMY $=$ AMY;

Struct (testnum). Raw_FS = FSd;

Struct (testnum). Raw TVP = TVP;

Struct (testnum). ChosenFd = FMd;

Struct (testnum). ChosenF1 = F1d;

Struct (testnum). ChosenF2 = F2d;

Struct (testnum). ChosenFY = FMdy;

Struct (testnum). ChosenFX $=$ FMdz;

Struct (testnum). ChosenTVP = TVPd;

Struct (testnum). TorqueY = ytorque;

Struct (testnum). Torquez = ztorque;

Struct (testnum). FilteredFY $=\mathrm{y}$;

Struct (testnum). FilteredFZ $=\mathrm{z}$;

Struct (testnum). FilteredYtorque = Ty;

Struct(testnum). Filteredztorque = Tz;

Struct (testnum). PhaseAvgY = PhasedTy;

Struct (testnum). PhaseAvgz = PhasedTz;

Struct (testnum). Lift $=$ Lift;

Struct (testnum). Thrust = Thrust;

Struct (testnum).ForceTime = FS time; \% time

Struct (testnum). PollTime = TVP time; \% time2

Struct (testnum). ThrustCoeff $=\bar{C} t$;

Struct (testnum). LiftCoeff $=\mathrm{Cl}$;

Struct (testnum). MinL = MinLift

Struct (testnum). MaxL = MaxLift

Struct (testnum). MinT = MinThrust;

Struct (testnum). MaxT = MaxThrust:

save ('PstTests.mat', 'Struct', '-append') 
Bibliography

Blevins, E., \& Lauder, G. V. (2013). Swimming near the substrate: a simple robotic model of stingray locomotion. Bioinspiration \& Biomimetics, 8: 016005, 112.

Eloy, C. (2012). Optimal Strouhal number for swimming animals. Journal of Fluids and Structures, 205-219.

Garcia, D. L., \& Katz, J. (2003). Trapped Vortex in Ground Effect. American Institute of Aeronautics and Astronautics Journal, Vol. 41, No. 4, 674-678.

Licht, S. (2008). Biomimetic Oscillating Foil Propulsion to Enhance Underwater Vehicle Agility and Maneuverability. Doctoral Dissertation, MIT, 216 pages.

Licht, S., \& Dahl, J. (2013). Thrust and lift generation in 'ground effect' for heaving and pitching high aspect ratio foils. 2013 Unmanned Untethered Submersible Technology Proceedings, Portsmouth, NH.

Polidoro, V. (2003). Flapping Foil Propulsion for Cruising and Hovering Autonomous Underwater Vehicles. Master's Thesis, MIT, 187 pages.

Rauworth, S. L. (2014). Development of an Underwater Flapping Foil Tow-Test System. Master's Thesis, URI, 89 pages.

Rayner, J. M. (1991). On the aerodynamics of animal flight in ground effect. Philosophical Transactions: Biological Sciences, Vol. 334, No. 1269, 119128.

Sfakiotakis, M., Lane, D. M., \& Davis, J. B. (1999). Review of Fish Swimming Modes for Aquatic Locomotion. IEEE Journal of Oceanic Engineering, 24, No.2, 237-253.

Streitlien, K., \& Triantafyllou, G. S. (1997). On Thrust Estimates For Flapping Foils. Journal of Fluids and Structures, 12, 47-55.

Techet, A. H. (2008). Propulsive performance of biologically inspired flapping foils at high Reynolds numbers. The Journal of Experimental Biology, 211, 274-279.

Wu, J., Shu, C., Zhao, N., \& Yan, W. (2014). Fluid dynamics of Flapping Insect Wing in Ground Effect. Journal of Bionic Engineering, 11, 52-60. 